\title{
Finite Energy Seiberg-Witten Moduli Spaces on 4-Manifolds Bounding Seifert Fibrations
}

\author{
LiviU I. NicOlaESCU
}

\begin{abstract}
We compute virtual dimensions of finite energy Seiberg-Witten moduli spaces on 4-manifolds bounding Seifert fibrations. The key moment is the determination of certain eta invariants. As an application of these computations we compute the Froyshov invariants of many Brieskorn homology spheres (e.g. $\Sigma(2,3,6 k \pm 1)$, $\Sigma(2,4 k+1,4 k+3), \Sigma(3,3 k+1,3 k+2))$. In turn, these lead to interesting topological results. For example, we prove that any negative definite 4-manifold bounding a Brieskorn homology sphere $\Sigma(2,3,6 k+1)$ must have diagonalizable intersection form.
\end{abstract}

\section{Introduction.}

The technical goal of the present paper is to compute the virtual dimensions of finite energy Seiberg-Witten moduli spaces for 4-manifolds bounding unions of Seifert fibrations. For cylinders over Seifert manifolds these moduli spaces describe tunnelings between the critical sets of the 3-dimensional Seiberg-Witten energy functional. These virtual dimensions were computed in [MOY] by identifying the space of tunnelings with an algebraic-geometric moduli space and then using a Riemann-Roch counting argument (see also [KMO] for a spectral flow approach).

However, we go beyond mere index theoretic computations and present some surprising topological implications of these computations. All the needed analytical baggage is almost entirely contained in [N1] and [N2] and we focus our attention mostly to the complex combinatorial and topological aspects due to the singular fibers of a Seifert fibration.

We will follow a strategy similar to the one we used in [N2] where we dealt with the slightly simpler problem when the 4-manifold bounds smooth $S^{1}$-bundles over Riemann surfaces. To compute the virtual dimension we will use the local description in [MMR] of such moduli spaces. The computation splits into two main steps. First, one needs to understand the critical sets of the 3-dimensional Seiberg-Witten functional on the boundary. This 
was accomplished in [MOY] and [N1] where the critical set was explicitly described and it was shown that (modulo some very explicit exceptions) they are Bott nondegenerate. This nondegeneracy is the best one can hope for since it essentially says that the asymptotics of a finite energy monopoles are determined by a linear equation. In particular, it allows us to avoid the introduction of thickened moduli spaces of [MMR] and work in the very convenient framework of exponentially weighted Sobolev spaces.

With this information in hand we can now proceed to compute these dimensions and the results of [MMR] reduce this problem to the computation of an Atiyah-Patodi-Singer (A-P-S) index for an operator which near the boundary has the form

$$
\mathcal{O}=\frac{\partial}{\partial t}-\tilde{\mathfrak{H}}
$$

Above, $t$ denotes the outgoing normal coordinate near the boundary. The operator $\tilde{\mathfrak{H}}$ further decomposes as

$$
\tilde{\mathfrak{H}}=\tilde{\mathfrak{H}}_{0}+\mathcal{P}
$$

where $\tilde{\mathfrak{H}}_{0}$ is a direct sum

$$
\tilde{\mathfrak{H}}_{0}=(\text { Odd signature operator }) \oplus(\text { a certain Dirac operator })
$$

and $\mathcal{P}$ is an explicit zeroth order perturbation of $\tilde{\mathfrak{H}}_{0}$. An excision argument reduces the computation of the A-P-S index of $\mathcal{O}$ to the computation of the A-P-S index of

$$
\mathcal{O}_{0}=\frac{\partial}{\partial t}-\tilde{\mathfrak{H}}_{0}
$$

and the computation of the spectral flow of the affine path $\tilde{\mathfrak{H}}_{0}+t P, 0 \leq$ $t \leq 1$. The perturbation analysis employed in [N2] for the computation of $S F\left(\tilde{\mathfrak{H}}_{0}+t \mathcal{P}\right)$ extends verbatim to the more general case of Seifert fibrations. We want only want to mention some special features of this computation.

First, $\tilde{\mathfrak{H}}_{0}+t P$ is not a Dirac operator. It is a complicated zero order perturbation of a direct sum and between a Dirac operator and the odd signature operator. Moreover, the eigenvalues contributing to the spectral flow cross the zero axis in a non-transversal manner and cannot be detected in first order approximation. One has to go further up the Taylor expansions of these eigenvalues, much like in [FL] and [KK]. There is an added difficulty since, unlike $[\mathrm{FL}]$ and $[\mathrm{KK}]$, the Taylor expansions cannot be expressed in purely topological terms. This is in stark contrast with the computations in 
[KMO] which involve only genuinely Dirac operators and eigenvalues crossing the axis transversally.

The eta invariant of $\tilde{\mathfrak{H}}_{0}$ is a sum of the eta invariant of the odd signature operator and the eta invariant of a certain Dirac operators. The eta invariant of the signature operator on Seifert fibrations is essentially a topological invariant and was computed in [O]. It can be expressed in terms of the classical Dedekind sums (see [HZ]) determined by the singular fibers and some topological quantities. The story is quite different for Dirac operators since in general their eta invariants are extremely sensitive to the background geometry.

We could not use the adiabatic approach in [N2] since we are not aware of an analogue of Bismut-Cheeger-Dai result for fibrations collapsing onto orbifolds. Instead, we follow a hands-on approach outlined in Appendix C of [N2] which relies on certain algebraic identities satisfied by the Dirac operators arising in Seiberg-Witten theory. We were able to describe the entire eta functions $\eta(s)$ of these operators in terms of Riemann-Hurwitz functions. The value $\eta(0)$ is then expressed in terms of Dedekind-Rademacher sums. (We refer to $[\mathrm{Ra}]$ for a presentation of these objects.)

When specialized to tunnelings, our virtual dimension formula looks quite different from the formula obtained in [MOY] which involves some Hirzebruch-Jung continued fractions. We did not directly proved the equality of these two descriptions (although one can conceivably use Rademacher's reciprocity law in $[\mathrm{Ra}]$ to achieve this) but numerical experimentations show perfect agreement.

It is perhaps worth emphasizing the differences between the present work and the closely related [MOY]. We provide information about the moduli spaces of finite energy monopoles on any cylindrical manifold bounding a Seifert manifold but we do not study the internal structure of individual monopoles. By contrast, the result in [MOY] provide almost complete information about the internal structure of an individual finite energy monopole on a special cylindrical manifold, but have very little to say about a general cylindrical manifold.

The eta invariant computations in this paper can be used in conjunction with the adiabatic analysis in [N1] to provide upper estimates for the Froyshov invariants $Y_{a, b, c}$ (introduced in $[\mathrm{Fr}]$ ) of the Brieskorn homology spheres $\Sigma(a, b, c)$. In many cases these estimates are optimal and have interesting topological consequences. For example, we prove the following result (Corollary 3.4, 3.9).

(i) $Y_{2,3,6 k+1}=Y_{2,4 k+1,4 k+3}=Y_{3,3 s+1,3 s+2}=0, Y_{2,3,6 k-1}=8$. 
(ii) $X$ is a 4-manifold with negative definite intersection form bounding a Brieskorn sphere $\Sigma(2,3,6 k+1)$ then the intersection form of $X$ must be diagonalizable. (The same result is true for $\Sigma(2,4 k+1,4 k+3)$ and $\Sigma(3,3 s+1,3 s+2)$ and it can be proved directly using Donaldson's first theorem and the fact proved in [CH] that these Brieskorn spheres bound contractible manifolds.)

(iii) If $X$ is a 4-manifold with negative definite intersection form $q$ which bounds a $\Sigma(2,3,6 k-1)$ and $q$ splits as $q_{1} \oplus q_{2}$ with $q_{2} \neq 0$ even, then $q_{2}=-E_{8}$ and $q_{1}$ is diagonalizable.

The equality $Y_{2,3,5}=8$ is proved in [Fr] relying on some very special geometric features of the Poincaré homology sphere $\Sigma(2,3,5)$.

The above result is not entirely obvious even when $X$ is the canonical plumbing associated to $\Sigma(2,3,6 k \pm 1)$. The plumbing diagrams are described in Figure 1, ?? in $\S 3.2$. We discovered in conversations with Ian Hambleton a simple combinatorial argument establishing these special facts directly (Remark 3.5).

As we have explained in $\S 3.2$, in the special cases $\Sigma(2,3,13), \Sigma(2,3,25)$ the property (ii) above is a consequence of Donaldson's Theorem A since these homology spheres bound contractible smooth manifolds. However, this argument fails for the Brieskorn spheres $\Sigma(2,3,6 k+1)$ with $k$ odd which cannot bound contractible smooth manifolds because their Rohlin invariant is nontrivial. They may bound rational homology balls (as does $\Sigma(2,3,7)$ ) in which case (ii) follows again from Donaldson's theorem, but it is not known whether is the case for all $k$.

The numerical experimentations suggest another beautiful phenomenon. In some sense (explained in §3.3) the Hirzebruch-Jung resolution of a Brieskorn sphere is the "most complicated" negative definite manifold it bounds. The results quoted above agree with this philosophy and moreover, work in progress shows this is also the case for lens spaces.

The paper is composed of three main sections. Subsection $\S 1.1$ lists the basic topological and geometric facts about Seifert manifolds used in the rest of the paper. In $\S 1.2$ we introduce the "adiabatic" Dirac operators which as shown in [MOY] and [N1] play an important role in the 3-dimensional theory. Most of this section is occupied with the computation of their eta invariants. We also explain how to obtain the eta invariants of the traditional Dirac operators (Remark 1.13).

The first half of Section 2 is a brief survey of the main results in [MMR] as they apply to our case. The virtual dimension formula (2.15) is then proved in $\S 2.3$. 
The third section is devoted to applications. We begin with a brief subsection in which we apply our formulæ to tunnelings. We have included it for two reasons. First, we solve a problem posed in [KMO], asking to express the virtual dimensions of the spaces of tunnelings in terms of Dedekind sums. Secondly, for topological applications, we need to know the dimensions of the spaces of tunnelings from a reducible monopole to an irreducible one. In [MOY] these computations were not explicitly carried out and they are indispensable in estimating the Froyshov invariants.

In the second half of this section we specialize to Brieskorn homology spheres $\Sigma(a, b, c)$. Using (in an essential fashion) the adiabatic analysis of [N1] and the explicit knowledge of the eta invariants we produce (often optimal !) upper bounds for the Froyshov invariants. These are then applied to obtain informations about the intersection forms of 4-manifolds bounding such homology spheres. We conclude with a speculative subsection where we formulate several conjectures suggested by numerical experiments.

Acknowledgments. I am indebted to Selman Akbulut, Ron Fintushel and Nikolai Saveliev for their interest and for generously sharing their expertise with me. The topological conversations with Ian Hambleton and Andy Nicas helped me better formulate, organize and structure my thoughts. I want to thank them and the Mathematics Department of the McMaster University for creating the right environment for me to develop and pursue the ideas in this paper.

Introduction . . . . . . . . . . . . . . . . . . . . 1027

1 Differential geometric preliminaries . . . . . . . . . . . 1032

$\S 1.1 \quad V$-bundles and Seifert fibrations . . . . . . . . 1032

$\S 1.2$ Eta invariants of Dirac operators on Seifert manifolds 1038

2 Finite energy Seiberg-Witten monopoles . . . . . . . . . . 1059

$\S 2.1$ The 4-dimensional Seiberg-Witten equations . . . . . 1060

$\S 2.2$ The 3-dimensional Seiberg-Witten equations . . . . 1063

$\S 2.3$ Virtual dimensions . . . . . . . . . . . . . . . . . . . . . . . . . . . . . . . . . . .

3 Applications . . . . . . . . . . . . . . . 1076

$\S 3.1$ Tunnelings . . . . . . . . . . . . . . . . 1076

$\S 3.2$ Froyshov invariants of Brieskorn spheres . . . . . . . . 1078

$\S 3.3$ Concluding remarks . . . . . . . . . . . . . . . . 1091

References . . . . . . . . . . . . . . . . . . . . . . 1093 


\section{Differential geometric preliminaries.}

\section{1. $V$-bundles and Seifert fibrations.}

In this paper, as in [FS] or [MOY], we will think of Seifert manifolds as sphere bundles determined by complex line $V$-bundles over Riemann $V$-surfaces.

The building blocks of $V$-manifolds (or orbifolds) are quotients of smooth manifolds determined by finite group actions A. $V$-manifold is obtained by gluing together such building blocks. More precisely, according to [Sa] a $V$-manifold of dimension $n$ is a collection

$$
M=\left(|M|,\left(U_{i}\right)_{i \in I},\left(\tilde{U}_{i}\right)_{i \in I},\left(G_{i}\right)_{i \in I},\left(\phi_{i}\right)_{i \in I},\left(\phi_{j i}\right)\right)
$$

with the following properties.

- $|M|$ is a Hausdorff space.

- $\left(U_{i}\right)_{i \in I}$ is an open cover of $|M|$ such that for any $x \in U_{i j}:=U_{i} \cap U_{j}$ there exists $k \in I$ so that $x \in U_{k} \subset U_{i j}$.

- $G_{i}$ denotes a finite group acting effectively on a connected open set $\tilde{U}_{i} \subset \mathbb{R}^{n}$. We assume the fixed point set of $G_{i}$ has dimension $\leq n-2$.

- $\phi_{i}$ is a $G_{i}$-invariant map $\tilde{U}_{i} \rightarrow U_{i}$ such that the induced map $\tilde{U}_{i} / G_{i} \rightarrow$ $U_{i}$ is a homeomorphisms.

- For any pair $(i, j)$ such that $U_{i} \subset U_{j}$ there exists a diffeomorphism $\phi_{j i}$ of $\tilde{U}_{i}$ onto an open subset of $\tilde{U}_{j}$ such that the diagram below is commutative

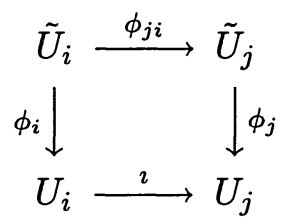

Remark 1.1. The effectiveness of the actions of the groups $G_{i}$ and the assumption on the fixed point set are usually not included in the definition of an orbifold. As shown in Lemmata 1 and 2 of [Sa] these imply that if $U_{i} \subset U_{j} \subset U_{k}$ then there exists $\gamma_{k i} \in G_{k}$ such that $\gamma_{k i} \phi_{k i}=\phi_{k j} \phi_{j i}$.

If $N=\left(|N|,\left(V_{\alpha}\right)_{\alpha \in A},\left(\tilde{V}_{\alpha}\right),\left(H_{\alpha}\right),\left(\psi_{\alpha}\right),\left(\psi_{\beta \alpha}\right)\right)$ is another $V$-manifold then a smooth $V$-map from $M$ to $N$ is a collection $\left(\varphi,\left(h_{i}\right)\right)$ with the following properties. 
- $\varphi$ is a map $I \rightarrow A$ such that $V_{\varphi(i)} \subset V_{\varphi(j)}$ if and only if $U_{i} \subset U_{j}$.

- For each $i \in I h_{i}$ is a smooth map $\tilde{U}_{i} \rightarrow \tilde{V}_{\varphi(i)}$ such that if $U_{i} \subset U_{j}$ the diagram bellow is commutative $(\alpha=\varphi(i), \beta=\varphi(j)$.

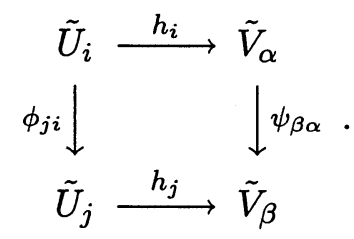

One can check that the collection $\left(h_{i}\right)$ does indeed induce a continuous map $h:|M| \rightarrow|N|$.

Let $G$ denote a Lie group acting on a smooth manifold $F$. A $V$-bundle with standard fiber $F$ and structure group $G$ consists of two $V$-manifolds

$$
\begin{gathered}
M=\left(|M|,\left(U_{i}\right)_{i \in I},\left(\tilde{U}_{i}\right)_{i \in I},\left(G_{i}\right)_{i \in I},\left(\Phi_{i}\right)_{i \in I},\left(\Phi_{j i}\right)\right) \\
B=\left(|B|,\left(V_{i}\right)_{i \in I},\left(\tilde{V}_{i}\right)_{i \in I},\left(G_{i}\right)_{i \in I},\left(\phi_{i}\right)_{i \in I},\left(\phi_{i j}\right)\right)
\end{gathered}
$$

and a smooth $V$-map $\pi=\left(\mathbf{1}_{I}, \pi_{i}\right): M \rightarrow B$ with the following properties.

- For every $i \in I$ the group $G_{i}$ acts on $F$ as well, its action commutes with the action of $G$ and there exists a $G_{i}$-equivariant homeomorphism

$$
\Psi_{i}: \tilde{U}_{i} \rightarrow \tilde{V}_{i} \times F
$$

- If $V_{i} \subset V_{j}$ there exists a smooth map $g_{j i}: \tilde{V}_{i} \rightarrow G$ such that

$$
\tilde{V}_{i} \times F \ni(v, f) \mapsto \Psi_{j} \circ \Psi_{j i} \circ \Psi_{i}^{-1}(v, f)=\left(v, g_{j i}(v) f\right) \in \tilde{V}_{j} \times F .
$$

Moreover, if $V_{i} \subset V_{j} \subset V_{k}$ then there exists $\gamma_{k i} \in G_{k}$ (as in Remark 1.1) such that $g_{k i}\left(\gamma_{k i} \phi_{k i}(p)\right)=g_{k j}\left(\phi_{j i}(p)\right) g_{j i}(p)$.

When $F$ is a vector space and the actions of $G$ and $G_{i}$ are linear the bundle is called a vector $V$-bundle. All the functorial concepts related to usual vector bundles (direct sum, tensor product etc.) have a $V$-counterpart. One can also speak of $V$-sections, $V$-metrics and $V$-connections in such vector $V$-bundles. All the above concepts have holomorphic counterparts defined in an obvious way.

Most of the standard operations of calculus can be carried out in the context of orbifolds as well. For example, the Chern-Weil theory has an 
immediate orbifold extension. We only want to mention here the concept of integration which as in the smooth case requires an orientability condition. An orbifold

$$
M=\left(|M|,\left(U_{i}\right)_{i \in I},\left(\tilde{U}_{i}\right)_{i \in I},\left(G_{i}\right)_{i \in I},\left(\phi_{i}\right)_{i \in I},\left(\phi_{i j}\right)\right)
$$

is said to be oriented if the sets $\tilde{U}_{i}$ are oriented, $G_{i}$ acts by orientation preserving maps and the gluing maps $\phi_{j i}$ are also orientation preserving. An $n$-form is a collection $\omega$ of $G_{i}$-invariant $n$-forms $\tilde{\omega}_{i}$ such that

$$
\phi_{j i}^{*} \tilde{\omega}_{j}=\tilde{\omega}_{i}, \quad \forall U_{i} \subset U_{j} .
$$

Using Remark 1.1 we deduce

$$
\phi_{k i}^{*} \tilde{\omega}_{k}=\phi_{j i}^{*} \phi_{k j}^{*} \tilde{\omega}_{k}
$$

so the above definitions are non-contradictory. Now choose a partition of unity $\left(\alpha_{i}\right)$ subordinated to the cover $\left(U_{i}\right)$ and define

$$
\int_{M} \omega=\sum_{i \in I} \frac{1}{\# G_{i}} \int_{\tilde{U}_{i}} \phi_{i}^{*}\left(\alpha_{i}\right) \tilde{\omega}_{i} .
$$

As in the smooth case one can verify the definition is independent of the various choices.

We will be interested only in compact, oriented 2-dimensional orbifolds without boundary and line $V$-bundles over them.

If $\Sigma$ is such an orbifold then the isotropy groups $G_{i}$ can only be cyclic, $G_{i} \cong \mathbb{Z}_{\alpha_{i}}, \alpha_{i}>2$. These have only isolated fixed points, $x_{1}, \ldots, x_{n}$. The underlying topological space is a smooth Riemann surface $|\Sigma|$. We can represent $\Sigma$ as a collection $(|\Sigma|, \vec{\alpha}, \vec{x})$ where $\vec{\alpha}=\left(\alpha_{1}, \ldots, \alpha_{n}\right)$ are the orders of isotropy and $\vec{x}=\left(x_{1}, \ldots, x_{n}\right)$ indicates the location of the singular points. The underlying topological space is completely described by the genus $g$ of $|\Sigma|$ so this orbifold is completely described by the collection $(g, \vec{\alpha}, \vec{x})$. We will use the notation $\Sigma(g, n ; \vec{\alpha}, \vec{x})$ but in general we will drop any information which is either irrelevant or clear from the context.

Suppose $L \rightarrow \Sigma(g ; \vec{\alpha}, \vec{x})$ is a line $V$-bundle. There is a new piece of information related to the singularity $\vec{x}$ namely the representation $\rho_{i}$ of $\mathbb{Z}_{\alpha_{i}}$ on the fiber $\mathbb{C}$. It can only be of the form $\rho_{i}\left(\exp \left(\frac{2 \pi \mathbf{i}}{\alpha_{i}}\right)\right)=\exp \left(\frac{2 \pi \beta_{i} \mathbf{i}}{\alpha_{i}}\right)$ where

$$
0 \leq \beta_{i}<\alpha_{i}, \quad i=1, \ldots, n \text {. }
$$


We again collect this information in the vector $\vec{\beta}$. It is important to emphasize that the isotropies $\vec{\beta}$ are normalized by the condition (1.1). We can now associate to $L$ its rational degree defined as

$$
\operatorname{deg} L=\int_{\Sigma} c_{1}(L) \in \mathbb{Q}
$$

As in the smooth case we have

$$
\operatorname{deg} L_{1} \otimes L_{2}=\operatorname{deg} L_{1}+\operatorname{deg} L_{2}
$$

and that is why we will often use the additive notation for the tensor product of line bundles.

Given an orbifold $\Sigma(\alpha, x)$ with a single singular point $x$ (of isotropy $\alpha$ ) we can construct a line $V$-bundle $H_{\alpha, x}$ with rational degree $1 / \alpha$ as follows. Let $|\Sigma|_{x}$ denote the surface obtained by deleting a small open disk $D_{x}$ around $x$. The total space of $H_{x}$ obtained by gluing $|\Sigma|_{x} \times \mathbb{C}$ to $\bar{D}_{x} \times \mathbb{C}$ via the map

$$
T_{\alpha}: S^{1} \times \mathbb{C} \cong \partial|\Sigma|_{x} \times \mathbb{C} \rightarrow \partial \bar{D}_{x} \times \mathbb{C} \cong S^{1} \times \mathbb{C}
$$

where

$$
T_{\alpha}(\exp (\mathbf{i} \theta), \rho \exp (\mathbf{i} \varphi))=(\exp (\mathbf{i}(-\alpha \theta+\varphi)), \rho \exp (\mathbf{i} \theta)) .
$$

Given a line bundle $L \rightarrow \Sigma(\vec{\alpha}, \vec{x})$ with singularity data $\vec{\beta}$ we can form

$$
|L|=L \otimes H_{x_{1}}^{-\beta_{1}} \otimes \cdots \otimes H_{x_{n}}^{-\beta_{n}} .
$$

One can show (see [FS]) that $|L|$ is a genuine smooth line bundle over $|\Sigma|$. Its degree is an integer and we have the following equality

$$
\operatorname{deg} L=\operatorname{deg}|L|+\sum_{i} \frac{\beta_{i}}{\alpha_{i}} .
$$

The rational degree and the singularity data completely determine the topological type of a line $V$-bundle. More precisely we have the following result (see $[\mathrm{FS}]$ for more details).

Proposition 1.2. Denote by $\operatorname{Pic}^{t}(\Sigma)$ the space of isomorphisms classes of line $V$-bundles over the orbifold $\Sigma(\vec{\alpha}, \vec{x})$. Define

$$
\tau=\tau_{\vec{\alpha}}: P i c^{t}(\Sigma) \rightarrow \mathbb{Q} \oplus \mathbb{Z}_{\alpha_{1}} \oplus \cdots \oplus \mathbb{Z}_{\alpha_{n}}
$$


by

$$
L(\vec{\beta}) \mapsto\left(\operatorname{deg} L, \beta_{1}\left(\bmod \alpha_{1}\right), \ldots, \beta_{n}\left(\bmod \alpha_{n}\right)\right)
$$

and $\delta: \mathbb{Q} \oplus \mathbb{Z}_{\alpha_{1}} \oplus \cdots \oplus \mathbb{Z}_{\alpha_{n}} \rightarrow \mathbb{Q} / \mathbb{Z}$ by

$$
\left(c, \beta_{1}\left(\bmod \alpha_{1}\right), \ldots, \beta_{n}\left(\bmod \alpha_{n}\right)\right) \mapsto\left(c-\sum_{i} \frac{\beta_{i}}{\alpha_{i}}\right)(\bmod \mathbb{Z}) .
$$

Then

$$
0 \rightarrow \operatorname{Pic}^{t}(\Sigma) \stackrel{\tau}{\rightarrow} \mathbb{Q} \oplus \mathbb{Z}_{\alpha_{1}} \oplus \cdots \oplus \mathbb{Z}_{\alpha_{n}} \stackrel{\delta}{\rightarrow} \mathbb{Q} / \mathbb{Z} \rightarrow 0
$$

is a short exact sequence of abelian groups.

The line bundle with singularity data $\vec{\beta}$ and rational degree $c$ will be denoted by $L(c, \vec{\beta})$.

A complex 2-orbifold $\Sigma(g ; \vec{\alpha})$ has an associated canonical line $V$-bundle $K_{\Sigma}=\Lambda^{1,0} T^{*} \Sigma$. Given a holomorphic line $V$-bundle $L=L(\vec{\beta})$ we can form the Dolbeault cohomology $H^{*}(L)$ and the Serre duality continues to hold

$$
H^{0}(L) \cong H^{1}\left(K_{\Sigma}-L\right), H^{1}(L) \cong H^{0}\left(K_{\Sigma}-L\right) .
$$

The Riemann-Roch theorem has an orbifold version due to Kawasaki [Kaw] $\left(h_{0}(L):=\operatorname{dim} H^{0}(L)\right)$

$$
h_{0}(L)-h_{0}\left(K_{\Sigma}-L\right)=1-g+\operatorname{deg}|L|=1-g+\operatorname{deg} L-\sum_{i} \frac{\beta_{i}}{\alpha_{i}} .
$$

In particular, if $L=|L|$ is the trivial line bundle we deduce from the Riemann Roch theorem that $h_{0}\left(K_{\Sigma}\right)=g$. Now, using the Riemann-Roch theorem for $K_{\Sigma}$ we deduce

$$
\operatorname{deg}\left|K_{\Sigma}\right|=2 g-2 .
$$

One can verify easily that the singularity data of $K_{\Sigma}$ are $\left(\alpha_{1}-1, \ldots, \alpha_{n}-1\right)$. Hence

$$
\operatorname{deg} K_{\Sigma}=2 g-2+\sum_{i}\left(1-\frac{1}{\alpha_{i}}\right) .
$$

The rational Euler characteristic of $\Sigma$ is defined as $\chi(\Sigma)=-\operatorname{deg} K_{\Sigma}$ from which we conclude

$$
\chi(\Sigma)=2-2 g-\sum_{i}\left(1-\frac{1}{\alpha_{i}}\right) .
$$


A Seifert manifold is by definition the unit sphere $V$-bundle of a complex line $V$-bundle over a 2-orbifold. More precisely, consider the 2-orbifold $\Sigma(g, \vec{\alpha})$ and the line $V$-bundle

$$
L(c, \vec{\beta}) \rightarrow \Sigma
$$

such that if $\beta_{i} \neq 0$ then g.c.d. $\left(\beta_{i}, \alpha_{i}\right)=1$. The unit sphere bundle $S(L)$ of $L$ is a Seifert manifold we will denote by $N(g ; b ; \vec{\alpha}, \vec{\beta})$ where $b=\operatorname{deg}|L|$. The collection $(g ; b ; \vec{\alpha}, \vec{\beta})$ is known as the (normalized) Seifert invariant. $\Sigma$ is known as the base of $N$.

The basic topological invariants of a Seifert manifold are known (see [FS]).

Theorem 1.3. If $N=N(g ; b, \vec{\alpha}, \vec{\beta}))$ ( $m$ singular points) then $\pi_{1}(N)$ admits the presentation

$$
\left\langle a_{i}, b_{i}, g_{i}, f, i=1, \ldots, g, j=1, \ldots, m\right.
$$

$$
\left.\left[a_{i}, f\right]=\left[b_{i}, f\right]=\left[g_{j}, f\right]=g_{j}^{\alpha_{j}} f^{-\beta_{j}}=f^{b} \prod\left[a_{i}, b_{i}\right] \prod g_{j}=1\right\rangle .
$$

where $f$ denotes the homotopy class of a regular fiber oriented by the action of $S^{1}$. The integral cohomology groups are given by

$$
H^{1}(N, \mathbb{Z})=\left\{\begin{aligned}
\mathbb{Z}^{2 g} & , \quad \operatorname{deg} \mathbb{L}_{0} \neq 0 \\
\mathbb{Z}^{2 g+1} & , \quad \operatorname{deg} \mathbb{L}_{0}=0
\end{aligned}\right.
$$

and

$$
H^{2}(N, \mathbb{Z}) \cong\left(P i c^{t}(\Sigma) / \mathbb{Z}[L]\right) \oplus \mathbb{Z}^{2 g}
$$

Moreover, the projection $\pi: N \rightarrow \Sigma$ pulls back line $V$-bundles on $\Sigma$ to genuine smooth line bundles on $N$ and the subgroup $\operatorname{Pic}^{t} / \mathbb{Z}[L]$ of $H^{2}(N, \mathbb{Z})$ can be identified with the image of the morphism

$$
\operatorname{Pic}^{t}(\Sigma) \stackrel{\pi^{*}}{\rightarrow}\{\text { Smooth line bundles on } N\} \stackrel{c_{1}}{\rightarrow} H^{2}(N, \mathbb{Z}) \text {. }
$$




\subsection{Eta invariants of Dirac operators on Seifert manifolds.}

In the sequel, we fix a 2-orbifold $\Sigma=\Sigma(g, m ; \vec{\alpha}, \vec{x})$ and a line $V$-bundle

$$
\mathbb{L}_{0}=\mathbb{L}_{0}(\ell, \vec{\beta}) \stackrel{\pi}{\rightarrow} \Sigma
$$

such that $0<\beta_{i}<\alpha_{i}$ and g.c.d. $\left(\alpha_{i}, \beta_{i}\right)=1, \forall i=1, \ldots, m$ and where $\ell$ the rational degree of $\mathbb{L}_{0}$. Denote by $N$ the associated Seifert manifold $N=S\left(\mathbb{L}_{0}\right)$. We orient it as the boundary of a complex manifold following the convention

$$
\text { outer normal } \wedge \text { or }(\text { boundary })=\text { or }(\text { manifold }) \text {. }
$$

As explained in [S], the manifold $N$ admits a locally homogeneous Riemann metric $g_{N}$. The natural $S^{1}$ action preserves such a metric. We denote by $\zeta$ the infinitesimal generator of this action. $\zeta$ is a nowhere vanishing Killing vector field. This metric induces a $V$-metric $g_{\Sigma}$ on the base which we normalize so that $\operatorname{vol}(\Sigma)=\pi . g_{\Sigma}$ has constant sectional curvature. By eventually rescaling the metric $g_{N}$ in the $\zeta$ direction we can assume $|\zeta|_{g_{N}} \equiv 1$. Now denote by $\varphi$ the global angular form defined as the $g_{N}$-dual of $\zeta$. As shown in [MOY] and [N1] we have a fundamental identity

$$
d \varphi=-2 \ell * \varphi .
$$

Denote by $C l\left(T^{*} N\right)$ the bundle of Clifford algebras generated by $\left(T^{*} N, g_{N}\right)$. The bundle $\Lambda^{*} T^{*} N$ is naturally a bundle of $C l\left(T^{*} N\right)$ modules (see [BGV]) and we denote by

$$
\text { c }: C l\left(T^{*} N\right) \rightarrow \operatorname{End}\left(\Lambda^{*} T^{*} N\right)
$$

the corresponding Clifford multiplication. The symbol map

$$
\sigma: C l\left(T^{*} N\right) \rightarrow \Lambda T^{*} N, u \mapsto \mathbf{c}(u) \cdot \mathbf{1},
$$

is a bundle isomorphism with inverse known as the quantization map. This allows us to define an action of $\Lambda T^{*} N$ on itself by

$$
\Lambda T^{*} N \rightarrow \operatorname{End}\left(\Lambda^{*} T^{*} N\right), \omega \mapsto \mathbf{c}\left(\sigma^{-1}(\omega)\right) .
$$

For simplicity we continue to denote this map with c. We call the resulting operation the Clifford multiplication by a form.

Let $\langle\varphi\rangle^{\perp}$ denote the orthogonal complement of the real line sub-bundle of $T^{*} N$ spanned by $\varphi$. As shown in [N1] the bundle $\langle\varphi\rangle^{\perp}$ is $\mathbf{c}(* \varphi)$-invariant. 
The bundle $\langle\varphi\rangle^{\perp}$ equipped with the (almost) complex structure $-\mathbf{c}(* \varphi)$ will be called the canonical line bundle of $N$ and will be denoted by $\mathfrak{K}$. We have the isomorphism

$$
\mathfrak{K} \cong \pi^{*} K_{\Sigma} .
$$

In [N1], $§ 2.2$ we showed that $\mathfrak{K}$ determines a canonical $\operatorname{spin}^{c}$ structure on $N$ with associated bundle of spinors

$$
\mathbb{S}_{c a n} \cong \mathfrak{K}^{-1} \oplus \mathbb{C}
$$

where $\underline{\mathbb{C}}$ denotes generically the trivial complex line bundle. This allows us to identify the space $\operatorname{Spin}^{c}(N)$ of $\operatorname{spin}^{c}$ structures on $N$ with the topological Picard group, $\mathrm{Pic}^{t}(N)$. The bundle of spinors corresponding to the line bundle $L \rightarrow N$ is

$$
\mathbb{S}_{L}=\mathbb{S}_{c a n} \otimes L \cong L \otimes \mathfrak{K}^{-1} \oplus L .
$$

As above, we get a Clifford multiplication map c $: \Lambda^{*} T^{*} N \rightarrow \operatorname{End}\left(\mathbb{S}_{L}\right)$. We "orient" it using the conventions of $[\mathrm{BC}]$

$$
\mathbf{c}\left(d v_{N}\right)=-\mathbf{i d} .
$$

On $T N$ there are two natural $g_{N}$-compatible connections. The first one is the Levi-Civita connection $\nabla^{L C}$. The other connection $\nabla^{\infty}$, called the adiabatic connection in [N1] and [N2] is described as follows. Using the decomposition

$$
T^{*} N=\langle\varphi\rangle \oplus\langle\varphi\rangle^{\perp} \cong\langle\varphi\rangle \oplus \pi^{*} T \Sigma
$$

we define $\nabla^{\infty}$ as the direct sum of the trivial connection on $\varphi$ and the pullback of the Levi-Civita connection of $T \Sigma$ on $\langle\varphi\rangle$.

The line bundle $\mathfrak{K}^{-1}$ comes with a natural hermitian connection induced by pullback from the Levi-Civita connection on the base. Thus a connection on $\operatorname{det} \mathbb{S}_{L} \cong L^{2} \oplus \mathfrak{K}^{-1}$ can be specified by indicating a connection on $L$. Fix such a connection $A$. Using the connection $\nabla^{L C}$ we obtain a Dirac operator $\mathfrak{D}_{A}$ on $\mathbb{S}_{L}$ while the adiabatic connection $\nabla^{\infty}$ induces a different Dirac operator, $D_{A}$. We will call $D_{A}$ the adiabatic Dirac operator. These two Dirac operators are related by the equality

$$
D_{A}=\mathfrak{D}_{A}+\frac{\ell}{2} \text {. }
$$


$D_{A}$ can be decomposed as

$$
D_{A}=Z_{A}+T_{A}
$$

where, with respect to the decomposition (1.8), the operators $Z$ and $T$ have the matrix descriptions

$$
Z_{A}=\left[\begin{array}{cc}
\mathbf{i} \nabla_{\zeta}^{A} & 0 \\
0 & -\mathbf{i} \nabla_{\zeta}^{A}
\end{array}\right]
$$

and

$$
T_{A}=\left[\begin{array}{cc}
0 & b \\
b & \bar{\partial}_{A} \\
\bar{\partial}_{A}^{*} & 0
\end{array}\right]
$$

We refer to [N1] for the exact definitions of $Z$ and $T$. It suffices to say that $Z$ uses only derivatives along the fiber direction while $T$ uses only derivatives along horizontal directions. Moreover $Z$ and $T$ interact in an especially nice manner:

$$
\{Z, T\}:=Z T+T Z=0 .
$$

The above equality is responsible (among many other things) for the following nice result.

Proposition 1.4. Consider a line $V$-bundle

$$
L=L(c, \vec{\gamma}) \rightarrow \Sigma=\Sigma(g, m ; \vec{\alpha}, \vec{x})
$$

equipped with a hermitian connection $A$. Denote by $\tilde{L}$ and resp. $\tilde{A}$ the pullbacks of $L$ and $A$ to $S\left(\mathbb{L}_{0}\right)$.

If $\ell=\operatorname{deg} \mathbb{L}_{0} \neq 0$ then the eta function $\eta_{L, A}(s)$ of the Dirac operator $D_{\tilde{A}}$ on $\mathbb{S}_{\tilde{L}}$ is given by

$$
\begin{aligned}
\eta_{L, A}(s)= & -2 \ell \zeta(s-1) \\
& +\sum_{i=1}^{m} \frac{1}{\alpha_{i}^{s}} \sum_{r=1}^{\alpha_{i}-1}\left(\left\{\frac{\gamma_{i}+r \beta_{i}}{\alpha_{i}}\right\}-\left\{\frac{\gamma_{i}-r \beta_{i}}{\alpha_{i}}\right\}\right) \zeta\left(s, r / \alpha_{i}\right) .
\end{aligned}
$$

The various quantities which appear in the above formula are defined as follows. $\zeta(s)$ is Riemann's zeta function, $\zeta(s, a)(a>0)$ denotes the RiemannHurwitz function

$$
\zeta(s, a)=\sum_{n=0}^{\infty} \frac{1}{(n+a)^{s}}
$$


and for each real number $x$ we have denoted by $\{x\}$ its fractional part defined by $\{x\} \in[0,1), x-\{x\} \in \mathbb{Z}$. In particular,

$$
\eta_{L, A}(0)=\frac{\ell}{6}-\sum_{i=1}^{m}\left(S_{i}^{+}-S_{i}^{-}\right)
$$

where $S_{i}^{ \pm}$denotes the sum

$$
S_{i}^{ \pm}=\sum_{r=1}^{\alpha_{i}}\left\{\frac{\gamma_{i} \pm r \beta_{i}}{\alpha_{i}}\right\}\left(\left(\frac{r}{\alpha_{i}}\right)\right), \quad((x)):=\left\{\begin{array}{rll}
\{x\}-\frac{1}{2} \quad \text { if } & x \in \mathbb{R} \backslash \mathbb{Z} \\
0 & \text { if } & x \in \mathbb{Z}
\end{array}\right.
$$

Proof. Define for $i=1, \ldots, m$ the $\alpha_{i}$-periodic function $G_{i}: \mathbb{R} \rightarrow \mathbb{R}$

$$
G_{i}(t)=-\left\{\frac{\gamma_{i}-t \beta_{i}}{\alpha_{i}}\right\}
$$

and set $G(t)=\sum_{i} G_{i}(t)$. Note that for any integer $n$ we have

$$
G(n)=\operatorname{deg}\left|L-n \mathbb{L}_{0}\right|-\operatorname{deg}\left(L-n \mathbb{L}_{0}\right) .
$$

Now set $F_{i}(n)=G_{i}(n)-G_{i}(-n), F(n)=G(n)-G(-n)$.

The connection $A$ on $L$ induces a holomorphic structure on $L$ and we denote by $\mathbb{L}$ the holomorphic line $V$-bundle thus obtained. The argument in the Appendix C of [N2] extends to the case of Seifert manifolds (because of Proposition 5.1.3 of [MOY]). We deduce (where $K=K_{\Sigma}$ )

$$
\begin{aligned}
& \eta_{L, A}(s)= \\
& \sum_{n=1}^{\infty} \frac{\left(h_{0}\left(K-\mathbb{L}-n \mathbb{L}_{0}\right)-h_{0}\left(\mathbb{L}+n \mathbb{L}_{0}\right)\right)+\left(h_{0}\left(\mathbb{L}-n \mathbb{L}_{0}\right)-h_{0}\left(K-\mathbb{L}+n \mathbb{L}_{0}\right)\right)}{n^{s}}
\end{aligned}
$$

(Riemann-Roch-Kawasaki)

$$
\begin{aligned}
& =\sum_{n=1}^{\infty} \frac{-\left(1-g+\operatorname{deg}\left|L+n \mathbb{L}_{0}\right|\right)+\left(1-g+\operatorname{deg}\left|L-n \mathbb{L}_{0}\right|\right)}{n^{s}} \\
& =\sum_{n=1}^{\infty} \frac{\operatorname{deg}\left|L-n \mathbb{L}_{0}\right|-\operatorname{deg}\left|L+n \mathbb{L}_{0}\right|}{n^{s}}
\end{aligned}
$$




$$
\begin{aligned}
& =\sum_{n=1}^{\infty} \frac{\operatorname{deg}\left(L-n \mathbb{L}_{0}\right)-\operatorname{deg}\left(L+n \mathbb{L}_{0}\right)+F(n)}{n^{s}} \\
& =\sum_{n=1}^{\infty} \frac{-2 n \operatorname{deg} \mathbb{L}_{0}+F(n)}{n^{s}} \\
& =-\sum_{n=1}^{\infty} \frac{2 \ell}{n^{s-1}}+\sum_{i=1}^{m} \sum_{n=1}^{\infty} \frac{G_{i}(n)-G_{i}(-n)}{n^{s}}
\end{aligned}
$$

At this point we use the following elementary lemma whose proof can be safely left to the reader.

Lemma 1.5. Suppose $f: \mathbb{R} \rightarrow \mathbb{R}$ is a periodic function of period $p \in \mathbb{Z}_{+}$. Then

$$
\sum_{n=1}^{\infty} \frac{f(n)}{n^{s}}=\sum_{r=1}^{p} \frac{f(r)}{p^{s}} \zeta(s, r / p) .
$$

Using the lemma we deduce

$$
\sum_{n=1}^{\infty} \frac{G_{i}(n)-G_{i}(-n)}{n^{s}}=\sum_{r=1}^{\alpha_{i}} \frac{G_{i}(r)-G_{i}(-r)}{\alpha_{i}^{s}} \zeta\left(s, r / \alpha_{i}\right) .
$$

The $\alpha_{i}$-th term in the second sum vanishes since $G_{i}\left(\alpha_{i}\right)=G_{i}\left(-\alpha_{i}\right)=$ $\left\{\gamma_{i} / \alpha_{i}\right\}$. The first part of the proposition is proved. The second part follows from the identities $\zeta(0, a)=1 / 2-a=-((a))(0<a<1), \zeta(0)=-1 / 12$ (see for example Chap. XIII of [WW]).

Remark 1.6. (a) The above result should be compared with the one in the smooth case (no singular fibers). Suppose we have different $V$ line bundles $L_{1}, L_{2}$ on $\Sigma$ equipped with connection $A_{1}, A_{2}$ which pullback to the same line bundle $\tilde{L}$ on $N$. Correspondingly we get two connections $\tilde{A}_{1}$ and $\tilde{A}_{2}$ and two adiabatic Dirac operators $D_{1}, D_{2}$. In the smooth case these operators have the same eta invariant which may not be the case when singular fibers are present. To put it differently, the eta function is sensible to the coupling connection. However, this dependence is mild if $A_{1}^{\prime}$ is another connection on $L_{1}$ then the Dirac operators $D_{1}^{\prime}$ associated to $\tilde{A}_{1}^{\prime}$ has the same eta function as $D_{1}$. The eta function "has only a vague idea" which $V$-line bundle on $\Sigma$ was used to construct the Dirac operator. Note that Serre duality implies immediately that $\eta_{L}(0)=\eta_{K-L}(0)$. One can verify this directly using the explicit description of the eta invariant. 
(b) The assumption $\ell \neq 0$ in Proposition 1.4 can be dropped although the above approach does not cover the case $\ell=0$. In this case $N$ can be represented as a quotient $\tilde{N} / \mathbb{Z}_{k}$ where $\tilde{N} \cong S^{1} \times N$ and $\mathbb{Z}_{k}$ acts freely and maps fibers to fibers (Thm. 5.4 in [S]). The identity (1.14) can be obtained as in Appendix C of [N2], working equivariantly on $\tilde{N}$.

The sum $S_{i}^{+}-S_{i}^{-}$can be expressed in terms of the Dedekind-Rademacher sums introduced in [Ra]. To see this note denote by $q_{i}$ the inverse of $\beta_{i}$ modulo $\alpha_{i}$ i.e. $\beta_{i} q_{i} \equiv 1\left(\bmod \alpha_{i}\right)$. Then

$$
\left\{\frac{\gamma_{i} \pm r \beta_{i}}{\alpha_{i}}\right\}=\left\{\begin{array}{ccc}
\left(\left(\frac{\gamma_{i} \pm r \beta_{i}}{\alpha_{i}}\right)\right)+\frac{1}{2} & \text { if } & r \not \equiv \mp q_{i} \gamma_{i}\left(\alpha_{i}\right) \\
\left(\left(\frac{\gamma_{i} \pm r \beta_{i}}{\alpha_{i}}\right)\right) & \text { if } & r \equiv \mp q_{i} \gamma_{i}\left(\alpha_{i}\right)
\end{array} .\right.
$$

We deduce

$$
S_{i}^{ \pm}=\sum_{r=1}^{\alpha_{i}}\left(\left(\frac{\gamma_{i} \pm r \beta_{i}}{\alpha_{i}}\right)\right)\left(\left(\frac{r}{\alpha_{i}}\right)\right)+\frac{1}{2} \sum_{r=1}^{\alpha_{i}}\left(\left(\frac{r}{\alpha_{i}}\right)\right) \pm \frac{1}{2}\left(\left(\frac{q_{i} \gamma_{i}}{\alpha_{i}}\right)\right) .
$$

The second sum vanishes since the function $x \mapsto((x))$ is odd. We deduce

$$
S_{i}^{ \pm}=\sum_{r=1}^{\alpha_{i}}\left(\left(\frac{\gamma_{i} \pm r \beta_{i}}{\alpha_{i}}\right)\right)\left(\left(\frac{r}{\alpha_{i}}\right)\right) \pm \frac{1}{2}\left(\left(\frac{q_{i} \gamma_{i}}{\alpha_{i}}\right)\right) .
$$

Using the notations in $[\mathrm{Ra}]$ we can rewrite

$$
S_{i}^{ \pm}=s\left( \pm \beta_{i}, \alpha_{i} ; \gamma_{i} / \alpha_{i}, 0\right) \pm \frac{1}{2}\left(\left(\frac{q_{i} \gamma_{i}}{\alpha_{i}}\right)\right)
$$

where

$$
s(\beta, \alpha ; x, y):=\sum_{r=1}^{\alpha}\left(\left(x+\beta \frac{r+y}{\alpha}\right)\right)\left(\left(\frac{r+y}{\alpha}\right)\right) .
$$

The entries $\alpha, \beta$ are coprime integers, $\alpha>0$ and $x, y \in \mathbb{R}$. Note that the above expression depends only on $x, y$ modulo $\mathbb{Z}$.

Thus the eta invariant of $D_{A}$ has the form

$$
\eta_{A}=\frac{\ell}{6}-2 S(\vec{\beta}, \vec{\alpha} ; \vec{\gamma})-d(\vec{\beta}, \vec{\alpha} ; \vec{\gamma})
$$


where

$$
\begin{aligned}
2 S(\vec{\beta}, \vec{\alpha}, \vec{\gamma}) & =\sum_{i=1}^{m}\left(s\left(\beta_{i}, \alpha_{i} ; \gamma_{i} / \alpha_{i}, 0\right)-s\left(-\beta_{i}, \alpha_{i} ; \gamma_{i} / \alpha_{i}, 0\right)\right) \\
& =2 \sum_{i=1}^{m} s\left(\beta_{i}, \alpha_{i} ; \gamma_{i} / \beta_{i}, 0\right)
\end{aligned}
$$

and

$$
d(\vec{\beta}, \vec{\alpha} ; \vec{\gamma})=\sum_{i=1}^{m}\left(\left(\frac{q_{i} \gamma_{i}}{\alpha_{i}}\right)\right) .
$$

The reason why we prefer this alternative description of $\eta$ is due to the Dedekind-Rademacher reciprocity law. To formulate it we must distinguish two cases.

- Both $x$ and $y$ are integers.

$$
s(\beta, \alpha ; x, y)+s(\alpha, \beta ; y, x)=-\frac{1}{4}+\frac{\alpha^{2}+\beta^{2}+1}{12 \alpha \beta} .
$$

- $x$ and/or $y$ is not an integer. Then

$$
\begin{aligned}
& s(\beta, \alpha ; x, y)+s(\alpha, \beta ; y, x) \\
& =((x)) \cdot((y))+\frac{\beta^{2} \psi_{2}(y)+\psi_{2}(\beta y+\alpha x)+\alpha^{2} \psi_{2}(x)}{2 \alpha \beta}
\end{aligned}
$$

where $\psi_{2}(x):=B_{2}(\{x\})$ and $B_{2}(z)$ is the second Bernoulli polynomial

$$
B_{2}(z)=z^{2}-z+\frac{1}{6}
$$

Denote by $R(\beta, \alpha ; x, y)$ the right hand side in the above reciprocity identities.

Note that $R(\alpha, \beta ; y, x)=R(\beta, \alpha ; x, y)$.

The reciprocity law coupled with the identities

$$
\begin{aligned}
s((\beta, 1 ; x, y) & =((\beta y+x)) \cdot((y)), \\
s(\beta, \alpha ; x, y) & =s(\beta-m \alpha, \alpha ; x+m y, y), \quad \forall m \in \mathbb{Z}
\end{aligned}
$$


and the Euclid's algorithm provides an efficient method of computing Dedekind Rademacher sums. For example,

$$
\begin{aligned}
s(4,7 ; 2 / 7,0)= & -s(7,4 ; 0,2 / 7)+R(4,7 ; 2 / 7,0) \\
= & -s(3,4 ; 2 / 7,2 / 7)+R(4,7 ; 2 / 7,0) \\
= & s(4,3 ; 2 / 7,2 / 7)-R(3,4 ; 2 / 7,2 / 7)+R(4,7 ; 2 / 7,0) \\
= & s(1,3 ; 4 / 7,2 / 7)-R(3,4 ; 2 / 7,2 / 7)+R(4,7 ; 2 / 7,0) \\
= & -s(3,1 ; 2 / 7,4 / 7)+R(1,3 ; 4 / 7,2 / 7)-R(3,4 ; 2 / 7,2 / 7) \\
& +R(4,7 ; 2 / 7,0)-((3 \cdot 4 / 7+2 / 7))((4 / 7))+R(1,3 ; 4 / 7,2 / 7) \\
& -R(4,3 ; 2 / 7,2 / 7)+R(4,7 ; 2 / 7,0) \\
= & R(1,3 ; 4 / 7,2 / 7)-R(4,3 ; 2 / 7,2 / 7)+R(4,7 ; 2 / 7,0) \\
= & -\frac{3}{28} .
\end{aligned}
$$

When dealing with reducible solutions of the 3-dimensional Seiberg-Witten equations we encounter Dirac operators coupled with flat connections.

We begin by classifying the topological types of such connections. The space of flat connections (modulo gauge transformations) can be identified via the holonomy representation with $\operatorname{Hom}\left(\pi_{1}(N), S^{1}\right) \cong \operatorname{Hom}\left(H_{1}(N), S^{1}\right)$ where for simplicity $H_{*}(X)$ (resp. $H^{*}(X)$ ) denotes the homology (resp. cohomology of $X$ ) with coefficients in $\mathbb{Z}$.

Given a representation $\rho: H_{1}(N) \rightarrow S^{1}$ we obtain a line bundle $L_{\rho}$ equipped with a flat connection. We get a map

$$
c_{1}: \operatorname{Hom}\left(H_{1}(N), S^{1}\right) \rightarrow \operatorname{Tors}\left(H^{2}(N)\right), \quad \rho \mapsto c_{1}\left(L_{\rho}\right) .
$$

Clearly, if two representations lie in the same component of $\operatorname{Hom}\left(H_{1}(N), S^{1}\right)$ then they determine isomorphic line bundles. Using the presentation (1.5) we see that the component of 1 in $\operatorname{Hom}\left(\pi_{1}(N), S^{1}\right)$ is the group $\operatorname{Hom}\left(\pi_{1}(|\Sigma|), S^{1}\right)$. We thus get a sequence

$$
1 \hookrightarrow \operatorname{Hom}\left(\pi_{1}(|\Sigma|), S^{1}\right) \stackrel{\pi^{*}}{\rightarrow} \operatorname{Hom}\left(\pi_{1}(N), S^{1}\right) \stackrel{c_{1}}{\rightarrow} \operatorname{Tors}\left(H^{2}(N)\right) \rightarrow 0
$$

in which $\pi^{*}$ (induced by the projection $\pi: N \rightarrow|\Sigma|$ ) is an injection, Range $\left(\pi^{*}\right) \subset \operatorname{ker} c_{1}$ and $c_{1}$ is onto.

Proposition 1.7. The sequence (1.19) is exact.

Proof. Set $\pi_{1}:=\pi_{1}(N)$. Since $c_{1}$ is a surjection and the group of components of $\operatorname{Hom}\left(\pi_{1}, S^{1}\right)$ is finite it suffices to show it has the same cardinality as Tors $\left(H^{2}(N)\right)$. 
For any groups $G, A$ ( $A$-abelian) denote by $H^{*}(G, A)$ the cohomology of $G$ with coefficients in the trivial $G$-module $A$. The short exact sequence

$$
0 \rightarrow \mathbb{Z} \rightarrow \mathbb{R} \rightarrow S^{1} \rightarrow 1
$$

leads to a long exact sequence in cohomology

$$
H^{1}\left(\pi_{1}, \mathbb{Z}\right) \stackrel{\imath}{\rightarrow} H^{1}\left(\pi_{1}, \mathbb{R}\right) \rightarrow H^{1}\left(\pi_{1}, S^{1}\right) \stackrel{\delta}{\rightarrow} H^{2}\left(\pi_{1}, \mathbb{Z}\right) \stackrel{\jmath}{\rightarrow} H^{2}\left(\pi_{1}, \mathbb{R}\right) .
$$

Using the isomorphisms $H^{1}\left(\pi_{1}, A\right) \cong \operatorname{Hom}\left(\pi_{1} /\left[\pi_{1}, \pi_{1}\right], A\right)$ for any abelian group $A$ (see for example [Mac]) and the presentation (1.5) we deduce

$$
\text { coker } \imath=\operatorname{Hom}\left(\pi_{1}(|\Sigma|), S^{1}\right)
$$

and thus we get a short exact sequence

$$
1 \hookrightarrow \operatorname{Hom}\left(\pi_{1}(|\Sigma|), S^{1}\right) \stackrel{\pi^{*}}{\rightarrow} \operatorname{Hom}\left(\pi_{1}(N), S^{1}\right) \rightarrow \operatorname{ker} \jmath \rightarrow 0 .
$$

Using the universal coefficient theorem we deduce that ker $\imath \cong \operatorname{Tors}\left(H^{2}\left(\pi_{1}\right)\right)$. To complete the proof of the proposition it suffices to show that

$$
\operatorname{Tors}\left(H^{2}\left(\pi_{1}\right)\right) \cong \operatorname{Tors}\left(H^{2}(N)\right) .
$$

Indeed, of the six Seifert geometries listed in [S], four of them live on contractible spaces. In these cases $N$ is a $K\left(\pi_{1}, 1\right)$ and we have a stronger, classical isomorphism $H^{2}\left(\pi_{1}\right) \cong H^{2}(N)$ (see [Mac]). The remaining two Seifert geometries are $S^{3}$ and $S^{2} \times \mathbb{R}$. If the universal cover is $S^{2} \times \mathbb{R}$, then the only compact, oriented Seifert manifold covered by $S^{2} \times \mathbb{R}$ are $S^{2} \times S^{1}$ and $\mathbb{R} P^{3} \# \mathbb{R} P^{3}$ (see $[\mathrm{S}]$ ) with fundamental groups $\mathbb{Z}$ and respectively $\mathbb{Z}_{2} * \mathbb{Z}_{2}$. Since $H^{2}(\mathbb{Z}) \cong H^{2}\left(S^{1}\right) \cong 0$ and $H^{2}\left(\mathbb{Z}_{2} * \mathbb{Z}_{2}\right)=\mathbb{Z}_{2} \oplus \mathbb{Z}_{2}$ ( Thm. VI.14.2 of [HS]) the isomorphism (1.20) is trivially satisfied.

Suppose now that $N$ is covered by $S^{3}$. Then $\pi_{1}$ is finite so that $H_{1}\left(\pi_{1}\right)$ is pure torsion and, using Cor. IV. 5.5 in [Mac], we get

$$
H^{2}\left(\pi_{1}\right) \cong \operatorname{Hom}\left(\pi_{1}, S^{1}\right) \cong \operatorname{Hom}\left(H_{1}\left(\pi_{1}\right), S^{1}\right) \cong H_{1}\left(\pi_{1}\right) \cong H_{1}(N) .
$$

The isomorphism (1.20) now follows from the Poincaré duality on $N$.

Let $\tilde{L} \rightarrow N$ denote a line bundle supporting a flat connection $A$. In particular, there exists a line $V$-bundle $L=L(c, \gamma) \rightarrow \Sigma$ which pulls back to $\tilde{L}$. $A$ defines a holonomy representation

$$
\operatorname{hol}_{A}: \pi_{1}(N) \rightarrow S^{1}
$$


and define $\theta=\theta_{A} \in[0,1)$ by the equality

$$
\exp (2 \pi \mathbf{i} \theta)=\operatorname{hol}_{A}(f)
$$

where $f$ denotes the homotopy class of a regular fiber of $N$. Consider the altered connection $B=A+\mathrm{i} \theta \varphi$. It has trivial holonomy along regular fibers. Using Proposition 5.1.3 in [MOY] we conclude that there exists a line $V$-bundle $L^{\prime}=L^{\prime}\left(c^{\prime} ; \vec{\gamma}^{\prime}\right) \rightarrow \Sigma$ and a $V$-connection $B^{\prime}$ on $L^{\prime}$ such that

$$
(\tilde{L}, B)=\pi^{*}\left(L^{\prime}, B^{\prime}\right) .
$$

In particular, $L(c, \vec{\gamma}) \equiv L^{\prime}\left(c^{\prime}, \vec{\gamma}^{\prime}\right)\left(\bmod \mathbb{L}_{0}\right)$ in $\operatorname{Pic}^{t}(\Sigma)$. Thus

$$
c^{\prime}-c \in \ell \mathbb{Z} \text {. }
$$

On the other hand $c^{\prime}=\operatorname{deg} L^{\prime}$ can be computed from

$$
c^{\prime}=\frac{\mathbf{i}}{2 \pi} \int_{\Sigma} F_{B^{\prime}}=\ell \theta
$$

Thus

$$
\ell \theta-c \in \ell \mathbb{Z}
$$

We distinguish two cases.

Case A. $\ell \neq 0$. In this case the equality (1.21) coupled with the restriction $0 \leq \theta<1$ uniquely determines $\theta$ by the equality

$$
\theta=\{c / \ell\} \text {. }
$$

Case B. $\ell=0$. In this case the the only pure torsion line bundle (which can support flats) are obtained via pullback from $V$-line bundles of zero rational degree. Assuming this is the case, the holonomy $\theta$ can have any value and in fact, any flat connection on $\tilde{L}$ is homotopic to one with trivial holonomy along fibers.

The next proposition summarizes the above considerations.

Proposition 1.8. (a) If $\ell \neq 0$ the quantity $\theta=\theta(\tilde{L}, A)$ is an invariant of the topological type of $\tilde{L}$ which we will denote by $\theta(\tilde{L})$. The correspondence

$$
P i c^{t}(\Sigma) / \mathbb{Z}\left[\mathbb{L}_{0}\right] \rightarrow S^{1}
$$


given by $L \mapsto \exp (2 \pi \mathbf{i} \theta(L))$ is a group morphism. Moreover, for any flat connection $A$ on $\tilde{L}$ and any $\theta \cong \theta(L)(\bmod \mathbb{Z})$ the connection $A+\mathbf{i} \theta \varphi$ is the pullback of a $V$-connection on the line $V$-bundle

$$
L_{\theta}=L+\left(\theta-\frac{c}{\ell}\right) \mathbb{L}_{0}
$$

(b) If $\ell=0$ there exist flat connections on $\tilde{L}$ iff $\tilde{L}$ is the pullback of a degree zero $V$-line bundle on $\Sigma$. In this case for any flat connection $A$ on $\tilde{L}$ the altered connection $A+\mathbf{i} \theta(A, \tilde{L}) \varphi$ is the pullback of some flat connection on a line $V$-bundle on the base.

Set again $\tilde{L}=\pi^{*}(L(c ; \vec{\gamma}))$. The spinor bundle $\mathbb{S}_{\tilde{L}}$ has determinant

$$
\operatorname{det} \mathbb{S}_{\tilde{L}}=\tilde{L}^{2} \otimes \mathfrak{K}^{-1} .
$$

$\mathfrak{K}$ comes equipped with a hermitian connection $\tilde{B}_{c a n}$ pulled back from a constant curvature connection $B_{c a n}$ on $K_{\Sigma}$ so that $\tilde{B}_{c a n}$ has trivial holonomy along fibers.

Any connection $\tilde{B}$ on $\tilde{L}$ induces a connection $\tilde{C}=\tilde{B}^{\otimes 2} \otimes \tilde{B}_{c a n}^{-1}$ on $\operatorname{det} \mathbb{S}_{\tilde{L}}$. We are interested in those connections $\tilde{B}$ such that the corresponding $\tilde{C}$ is flat. This is equivalent to solving (modulo gauge transformations of $\tilde{L}$ ) the equation

$$
F_{\tilde{B}}=\frac{1}{2} F_{\tilde{B}_{c a n}} .
$$

Denote by $\mathcal{R}(\tilde{L})$ the space of gauge equivalence classes of $\tilde{B}^{\prime} s$ satisfying (1.23). We again consider separately two cases.

Case 1. $\quad \ell \neq 0$. It is very easy to construct a particular solution of (1.23). It suffices to pick a constant curvature connection $A_{0}$ on $L(c ; \vec{\gamma})$. Denote its pullback to $N$ by $\tilde{A}_{0}$. Since $\operatorname{vol}(\Sigma)=\pi$ we deduce

$$
F_{\tilde{A}_{0}}=\frac{2 c}{\mathbf{i}} \pi^{*}\left(d v_{\Sigma}\right)=\frac{\mathbf{i} c}{\ell} d \varphi
$$

It is clear now that the connection

$$
\tilde{B_{0}}=\tilde{A}_{0}+\mathrm{i} \frac{\operatorname{deg} K_{\Sigma}-2 c}{2 \ell} \varphi
$$

satisfies (1.23). 
If $\tilde{B} \in \mathcal{R}(\tilde{L})$ is another solution then the connection

$$
\tilde{B} / \tilde{B}_{0}:=\tilde{B} \otimes \tilde{B}_{0}^{\otimes-1}
$$

on the trivial bundle is flat. Thus any solution $\tilde{B}$ of (1.23) can be represented as

(1.24) $\tilde{B}=\tilde{B}_{0} \otimes($ flat connection on the trivial bundle $)$

$$
=\tilde{A}_{0} \otimes(\text { flat connection on the trivial bundle })+\mathrm{i} \frac{\operatorname{deg} K_{\Sigma}-2 c}{2 \ell} \varphi \text {. }
$$

The above description has an ambiguity built-in stemming from a choice of a line $V$-bundle bundle $L$ on $\Sigma$ which pulls back to $\tilde{L}$. We can now be more precise about this choice.

Definition 1.9. Let $N \stackrel{\pi}{\rightarrow} \Sigma$ denote a Seifert fibration over a 2-orbifold $\Sigma$ such that $\ell=\operatorname{deg}(N) \neq 0$. The canonical representative of a line bundle $\tilde{L} \rightarrow N \in \pi^{*}\left(\operatorname{Pic}^{t}(\Sigma)\right)$ is the line $V$-bundle $L=L(c ; \gamma)$ uniquely defined by the requirements $\pi^{*}(L) \cong \tilde{L}$ and

$$
\rho(\tilde{L}):=\frac{\operatorname{deg} K_{\Sigma}-2 c}{2 \ell} \in[0,1) .
$$

A canonical connection on $\tilde{L}$ is the pullback of a connection $A_{0}$ on the canonical representative which has constant curvature

$$
F_{A_{0}}=\text { const. } d v_{\Sigma}
$$

We can rephrase (1.24) by saying that any solution of (1.23) has the form (1.25) canonical connection $\otimes$ flat on the trivial bundle $+\mathbf{i} \rho(\tilde{L}) \varphi$.

We immediately see the meaning of $\rho(\tilde{L}): \exp (2 \pi \mathbf{i} \rho)$ is the holonomy along a nonsingular fiber of an arbitrary connection $\tilde{B} \in \mathcal{R}$.

Case 2. $\ell=0$ Note first that $c_{1}(\mathfrak{K})$ may not be a torsion class. However there exist line $V$-bundle $L$ on $\Sigma$ such that $c_{1}\left(\pi^{*} L^{2} \otimes \mathfrak{K}^{-1}\right)$ is torsion. This is equivalent to

$$
2 \operatorname{deg} L=\operatorname{deg} K_{\Sigma}, \quad L \in \operatorname{Pic}^{t}(\Sigma)
$$


If now $\tilde{L}$ is the pullback of a solution $L$ of (1.26) we can argue as in the case $\ell \neq 0$ to see that once we determine a particular solution of (1.23) any other can be obtaining by a twist with a flat connection on the trivial line bundle. The problem is one of existence. Pick any constant curvature connection $A_{0}$. Then its pullback to $\tilde{L}$ will satisfy (1.23).

The above structural description of the flat connections on a Seifert manifold will enable us to compute the eta functions of Dirac operators coupled with such connections. More precisely, we have the following result.

Proposition 1.10. Consider an oriented orbifold $\Sigma(g, m ; \vec{\alpha})$ with $m$ singular points and let $\mathbb{L}_{0}$ denote the line $V$-bundle $\mathbb{L}_{0}=L(\ell ; \vec{\beta})$ of degree $\ell \neq 0$ such that $\alpha_{i}, \beta_{i}$ are coprime $\forall i$. Denote by $N$ the associated unit sphere bundle equipped with the geometry described at the beginning of this section. Let $\tilde{L} \in \pi^{*} \operatorname{Pic}^{t}(\Sigma)$ be a line bundle on $N$ with torsion $c_{1}$. Denote by $L=L(c, \vec{\gamma}) \rightarrow \Sigma$ its canonical representative. denote another $V$-line bundle over $\Sigma$ and denote by $\tilde{L}$ its pullback to $N$. Equip it with a connection $\tilde{B} \in \mathcal{R}$ of the form

$$
\tilde{B}=\tilde{B}_{0}+\mathbf{i} \rho \varphi
$$

where $\rho=\rho(\tilde{L})=\frac{\operatorname{deg} K-2 c}{2 \ell}$ and $\tilde{B}_{0}$ is the pullback of a constant curvature connection $B_{0}$ on $L$. Denote by $\eta_{L}(s)=\eta_{\tilde{L}, \tilde{B}}(s)$ the eta function of the associated adiabatic Dirac operator $D_{\tilde{B}}$ on $\mathbb{S}_{\tilde{L}}$.

(a) If $\rho=0$ then

$$
\begin{aligned}
& \eta_{L}(s)=-2 \ell \zeta(s-1) \\
& \quad+\sum_{i=1}^{m} \frac{1}{\alpha_{i}^{s}} \sum_{r=1}^{\alpha_{i}-1}\left(\left\{\frac{\gamma_{i}+r \beta_{i}}{\alpha_{i}}\right\}-\left\{\frac{\gamma_{i}-r \beta_{i}}{\alpha_{i}}\right\}\right) \zeta\left(s, r / \alpha_{i}\right) .
\end{aligned}
$$

(b) If $\rho \in(0,1)$ then

$$
\begin{aligned}
& \text { (1.27) } \eta_{L}(s)=\frac{\operatorname{deg} K-\operatorname{deg}|K|}{2}(\zeta(s, \rho)-\zeta(s, 1-\rho)) \\
& -\sum_{i=1}^{m} \frac{1}{\alpha_{i}^{s}} \sum_{k=0}^{\alpha_{i}-1}\left\{\frac{\gamma_{i}-k \beta_{i}}{\alpha_{i}}\right\}\left(\zeta\left(s,\left\{\frac{k+\rho}{\alpha_{i}}\right\}\right)-\zeta\left(s, 1-\left\{\frac{k+\rho}{\alpha_{i}}\right\}\right)\right) \\
& -\ell \zeta(s-1, \rho)-\ell \zeta(s-1,1-\rho) .
\end{aligned}
$$


In particular, for $\rho=0$

$$
\eta_{L}(0)=\frac{\ell}{6}-2 S(\vec{\beta}, \vec{\alpha}, \vec{\gamma})-d(\vec{\beta}, \vec{\alpha}, \vec{\gamma})
$$

while if $0<\rho<1$

$$
\begin{aligned}
\eta_{L}(0)= & \frac{\operatorname{deg} K-\operatorname{deg}|K|}{2}(1-2 \rho) \\
& -\sum_{i=1}^{m} \sum_{k=0}^{\alpha_{i}-1}\left\{\frac{\gamma_{i}-k \beta_{i}}{\alpha_{i}}\right\}\left(1-2\left\{\frac{k+\rho}{\alpha_{i}}\right\}\right)-\ell \rho(1-\rho)+\frac{\ell}{6}
\end{aligned}
$$

Proof. The case $\rho=0$ is contained in Proposition 1.4 so we will consider only the case of fractional holonomy $0<\rho<1$. We will follow the same arguments and notations as in the Appendix $\mathrm{C}$ of [N2] and we deduce

$$
\eta(s)=\sum_{\mu>0} \frac{\operatorname{dim} F_{\mu}-\operatorname{dim} F_{-\mu}}{\mu^{s}}
$$

where for any $\mu \in \mathbb{R}$ we denoted by $F_{\mu}$ the space of pairs

$$
\alpha \oplus \beta \in C^{\infty}(\tilde{L}) \oplus C^{\infty}\left(\tilde{L} \otimes \mathfrak{K}^{-1}\right)
$$

satisfying

$$
\begin{aligned}
& \mathbf{i} \nabla_{\zeta}^{\tilde{B}} \alpha=\mu \alpha, \quad \bar{\partial}_{\tilde{B}}^{*} \alpha=0, \\
& -\mathbf{i} \nabla_{\zeta}^{\tilde{B}} \beta=\mu \beta, \quad \bar{\partial}_{\tilde{B}} \beta=0 .
\end{aligned}
$$

Denote by $f_{\mu}^{-}$(resp. by $f_{\mu}^{+}$) the dimension of the space of solutions of (1.30) (resp. (1.31)). We can rewrite (1.29) as

$$
\eta(s)=\sum_{\mu \neq 0} \frac{\operatorname{sign}(\mu) f_{\mu}^{+}}{|\mu|^{s}}+\sum_{\mu \neq 0} \frac{\operatorname{sign}(\mu) f_{\mu}^{-}}{|\mu|^{s}} .
$$

We will only show how to determine $f_{\mu}^{+}$since the determination of $f_{\mu}^{-}$is entirely similar.

Set for simplicity $\tilde{B}_{\mu}^{ \pm}=\tilde{B} \mp \mathbf{i} \mu \varphi$. Note first that $\bar{\partial}_{\tilde{B}} \beta=\bar{\partial}_{\tilde{B}_{\mu}^{ \pm}} \beta$ since the transition $\tilde{B} \rightarrow \tilde{B}_{\mu}^{ \pm}$does not alter the derivatives along horizontal directions. On the other hand, the equation $-\mathbf{i} \nabla_{\zeta}^{\tilde{B}} \beta=\mu \beta$ can be rewritten as

$$
-\mathbf{i} \nabla_{\zeta}^{\tilde{B}_{\mu}^{+}} \beta=0 .
$$


Thus the equations (1.31) are equivalent to

$$
-\mathbf{i} \nabla_{\zeta}^{\tilde{B}_{\mu}^{+}} \beta=0, \quad \bar{\partial}_{\tilde{B}_{\mu}^{+}} \beta=0 .
$$

If (1.33) admits a nontrivial solution $\beta$ then $\beta$ must be $\tilde{B}_{\mu}^{+}$-covariant constant along the fibers. This implies that the pair $\left(\pi^{*} L, \tilde{B}_{\mu}^{+}\right)$is the pullback of a pair (line bundle $L_{\mu}$, connection $B_{\mu}$ on $L_{\mu}$ ) on the base $\Sigma$. Using the equality $\tilde{B}_{\mu}^{+}=\tilde{B}_{0}+(\rho-\mu) \mathbf{i} \varphi$ we can determine the curvature of the connection $B_{\mu}$ from

$$
F_{B_{\mu}}=F_{B_{0}}+2 \ell(\mu-\rho) \mathbf{i} d v_{\Sigma}
$$

so that

$$
\operatorname{deg} L_{\mu}=\operatorname{deg} L-\ell(\mu-\rho)=c-(\mu-\rho) .
$$

Thus $\mu-\rho \in \mathbb{Z}$ since $\pi^{*} L_{\mu}=\pi^{*} L$. If we set $n=\mu-\rho$ we deduce $L_{\mu}=$ $L-n \mathbb{L}_{0}$. The connection $B_{\mu}$ induces a holomorphic structure on $L_{\mu}$ and we can now identify $F_{\mu}^{+}$with the space of holomorphic sections of $L_{\mu}$. We have thus proved

$$
f_{\mu}^{+} \neq 0 \Rightarrow \mu=n+\rho, n \in \mathbb{Z}, f_{\mu}^{+}=h_{0}\left(L-n \mathbb{L}_{0}\right) .
$$

Similarly we deduce

$$
f_{\mu}^{-} \neq 0 \Rightarrow \mu=n-\rho, n \in \mathbb{Z}, f_{\mu}^{-}=h_{0}\left(K-L-n \mathbb{L}_{0}\right) .
$$

Using these informations in (1.32) we conclude

$$
\begin{aligned}
\eta(s) & =\sum_{n \in \mathbb{Z}} \frac{\operatorname{sign}(n+\rho) h_{0}\left(L-n \mathbb{L}_{0}\right)}{|n+\rho|^{s}}+\sum_{n \in \mathbb{Z}} \frac{\operatorname{sign}(n-\rho) h_{0}\left(K-L-n \mathbb{L}_{0}\right)}{|n-\rho|^{s}} \\
& =\sum_{n \in \mathbb{Z}} \frac{\operatorname{sign}(n+\rho)\left(h_{0}\left(L-n \mathbb{L}_{0}\right)-h_{0}\left(K-L+n \mathbb{L}_{0}\right)\right)}{|n+\rho|^{s}}
\end{aligned}
$$

(use Riemann-Roch-Kawasaki)

$$
=\sum_{n \in \mathbb{Z}} \frac{\operatorname{sign}(n+\rho)\left(-\frac{1}{2} \operatorname{deg}|K|+\operatorname{deg}\left|L-n \mathbb{L}_{0}\right|\right)}{|n+\rho|^{s}}
$$

( $c=\operatorname{deg} L, G(n)$ as in the proof of Proposition 1.4)

$$
=\sum_{n \in \mathbb{Z}} \frac{\left.\operatorname{sign}(n+\rho)((\operatorname{deg} K-\operatorname{deg}|K|) / 2)+G(n)-\frac{1}{2} \operatorname{deg} K+c-n \ell\right)}{|n+\rho|^{s}}
$$


$\left(\ell \rho=\frac{1}{2} \operatorname{deg} K-c\right)$

$$
\begin{gathered}
=\sum_{n \in \mathbb{Z}} \frac{\operatorname{sign}(n+\rho)((\operatorname{deg}(K-\operatorname{deg}|K|) / 2+G(n))}{|n+\rho|^{s}} \\
-\ell \sum_{n \in \mathbb{Z}} \frac{\operatorname{sign}(n+\rho)(n+\rho)}{|n+\rho|^{s}}
\end{gathered}
$$

$$
\begin{aligned}
\left(S_{\rho}:=\right. & \rho+\mathbb{Z}) \\
= & \frac{1}{2}(\operatorname{deg} K-\operatorname{deg}|K|) \sum_{\mu \in S_{\rho}} \frac{\operatorname{sign}(\mu)}{|\mu|^{s}} \\
& \quad+\sum_{i=1}^{m} \sum_{\mu \in S_{\rho}} \frac{\operatorname{sign}(\mu) G_{i}(\mu-\rho)}{|\mu|^{s}}-\ell \sum_{\mu \in S_{\rho}} \frac{1}{|\mu|^{s-1}} \\
= & : \mathbf{S}_{1}+\mathbf{S}_{2}+\mathbf{S}_{3} .
\end{aligned}
$$

To compute the first two sums we use the following elementary result.

Lemma 1.11. For any periodic function $f: \mathbb{R} \rightarrow \mathbb{R}$ of period $p \in \mathbb{Z}_{+}$and any $\rho \in(0,1)$ define

$$
\eta(s, f, \rho):=\sum_{\mu \in S_{\rho}} \frac{\operatorname{sign}(\mu) f(\mu-\rho)}{|\mu|^{s}} .
$$

Then

$$
\eta(s, f, \rho)=\sum_{k=0}^{p-1} \frac{f(k)}{p^{s}}\left(\zeta\left(s,\left\{\frac{k+\rho}{p}\right\}\right)-\zeta\left(s, 1-\left\{\frac{k+\rho}{p}\right\}\right)\right) .
$$

Proof of the lemma. For any $\rho \in \mathbb{R} \backslash \mathbb{Z}$ set

$$
S_{\rho}^{ \pm}=\left\{x \in S_{\rho} ; \pm x>0\right\}
$$


We have

$$
\begin{aligned}
\eta(s, f, \rho) & =\sum_{k=0}^{p-1} \frac{f(k)}{p^{s}} \sum_{n \in \mathbb{Z}} \frac{\operatorname{sign}(n p+k+\rho)}{|n+(k+\rho) / p|^{s}}=\sum_{k=0}^{p-1} \frac{f(k)}{p^{s}} \sum_{\mu \in S_{(k+\rho) / p}} \frac{\operatorname{sign}(\mu)}{|\mu|^{s}} \\
& =\sum_{k=0}^{p-1} \frac{f(k)}{p^{s}}\left(\sum_{\mu \in S_{(k+\rho) / p}^{+}} \frac{1}{|\mu|^{s}}\right)-\sum_{k=0}^{p-1} \frac{f(k)}{p^{s}}\left(\sum_{\mu \in S_{(k+\rho) / p}^{-}} \frac{1}{|\mu|^{s}}\right) \\
& =\sum_{k=0}^{p-1} \frac{f(k)}{p^{s}}\left(\zeta\left(s,\left\{\frac{k+\rho}{p}\right\}\right)-\zeta\left(s, 1-\left\{\frac{k+\rho}{p}\right\}\right)\right) .
\end{aligned}
$$

Using Lemma 1.11 we deduce

$$
\mathbf{S}_{1}=\frac{\operatorname{deg} K-\operatorname{deg}|K|}{2}(\zeta(s, \rho)-\zeta(s, 1-\rho))
$$

and (since $G_{i}$ is $\alpha_{i}$-periodic)

$$
\mathbf{S}_{2}=\sum_{i=1}^{m} \sum_{k=0}^{\alpha_{i}-1} \frac{G_{i}(k)}{\alpha_{i}^{s}}\left(\zeta\left(s,\left\{\frac{k+\rho}{\alpha_{i}}\right\}\right)-\zeta\left(s, 1-\left\{\frac{k+\rho}{\alpha_{i}}\right\}\right)\right)
$$

Finally, the third sum can be written as

$$
\mathbf{S}_{3}=-\ell(\zeta(s-1, \rho)+\zeta(s-1,1-\rho)) .
$$

To compute $\eta_{L}(0)$ we only need to use the classical formulæ (see [WW])

$$
\zeta(0, a)=\frac{1}{2}-a, \zeta(-1, a)=-\frac{1}{12}+\frac{1}{2} a(1-a) \quad \forall a>0
$$

which imply $\zeta(0, a)-\zeta(0,1-a)=1-2 a$. The proposition is proved.

Remark 1.12. (a) The formula we have just proved illustrates another surprising feature of the eta invariant. The choice of $\vec{\gamma}$ in the above proposition is uniquely determined by $c_{1}(\tilde{L})$. This shows the eta invariant of an adiabatic Dirac coupled with a flat connection is a topological quantity!

(b) The above results extend to the case $\ell=0$. In this case $\rho$ should be defined as half the "logarithm" of the holonomy of the twisting connection along a regular fiber. 
As in Proposition 1.4 we can express the eta invariants in (1.28) in terms of Dedekind-Rademacher sums. To do this observe that

$$
\begin{aligned}
& \sum_{k=0}^{\alpha_{i}-1}\left\{\frac{\gamma_{i}-k \beta_{i}}{\alpha_{i}}\right\}\left(1-2\left\{\frac{k+\rho}{\alpha_{i}}\right\}\right) \\
= & \sum_{k=0}^{\alpha_{i}-1}\left(\left(\left(\frac{\left.\gamma_{i} \beta_{i} \equiv 1 \bmod \alpha_{i}\right)}{\alpha_{i}}\right)\right)+1 / 2\right)\left(1-2\left\{\frac{k+\rho}{\alpha_{i}}\right\}\right)-\frac{1}{2}\left(1-2\left\{\frac{q_{i} \gamma_{i}+\rho}{\alpha_{i}}\right\}\right) . \\
& \sum_{k=0}^{\alpha_{i}-1}\left(\left(\frac{\gamma_{i}-k \beta_{i}}{\alpha_{i}}\right)\right)\left(1-2\left\{\frac{k+\rho}{\alpha_{i}}\right\}\right) \\
& +\frac{1}{2} \sum_{k=0}^{\alpha_{i}-1}\left(1-2\left\{\frac{k+\rho}{\alpha_{i}}\right\}\right)-\frac{1}{2}\left(1-2\left\{\frac{q_{i} \gamma_{i}+\rho}{\alpha_{i}}\right\}\right) \\
= & \sum_{k=0}^{\alpha_{i}-1}\left(\left(\frac{\gamma_{i}-k \beta_{i}}{\alpha_{i}}\right)\right)\left(1-2\left\{\frac{k+\rho}{\alpha_{i}}\right\}\right) \\
& +\frac{\alpha_{i}-1}{2}+\left\{\frac{q_{i} \gamma_{i}+\rho}{\alpha_{i}}\right\}-\sum_{k=0}^{\alpha_{i}-1}\left\{\frac{k+\rho}{\alpha_{i}}\right\} .
\end{aligned}
$$

The last sum is equal to $\rho+\frac{\alpha_{i}-1}{2}$ and thus we deduce

$$
\begin{aligned}
& \sum_{k=0}^{\alpha_{i}-1}\left\{\frac{\gamma_{i}-k \beta_{i}}{\alpha_{i}}\right\}\left(1-2\left\{\frac{k+\rho}{\alpha_{i}}\right\}\right) \\
& =-\rho+\left\{\frac{q_{i} \gamma_{i}+\rho}{\alpha_{i}}\right\}+\sum_{k=0}^{\alpha_{i}-1}\left(\left(\frac{\gamma_{i}-k \beta_{i}}{\alpha_{i}}\right)\right)\left(1-2\left\{\frac{k+\rho}{\alpha_{i}}\right\}\right)
\end{aligned}
$$

The last sum (denote it temporarily by $S$ ) can be expressed in terms of Dedekind-Rademacher sums. More precisely, since $0<\rho<1$ is not an integer then

$$
S=2 \sum_{k=0}^{\alpha_{i}-1}\left(\left(\frac{k \beta_{i}-\gamma_{i}}{\alpha_{i}}\right)\right) \cdot\left(\left(\frac{k+\rho}{\alpha_{i}}\right)\right)=2 s\left(\beta_{i}, \alpha_{i} ; \frac{\gamma_{i}+\beta_{i} \rho}{\alpha_{i}},-\rho\right) .
$$

For $\rho \in(0,1)$ and $\beta q \equiv 1 \bmod \alpha$ define

$$
F_{\rho}(\alpha, \beta, \gamma):=\left\{\frac{q \gamma+\rho}{\alpha}\right\}
$$


Then

$$
\begin{aligned}
\eta(0)=\frac{1}{2}(\operatorname{deg} K & -\operatorname{deg}|K|)(1-2 \rho)-\ell \rho(1-\rho)+\frac{\ell}{6}+m \rho \\
& -2 \sum_{i=1}^{m} s\left(\beta_{i}, \alpha_{i} ; \frac{\gamma_{i}+\beta_{i} \rho}{\alpha_{i}},-\rho\right)-\sum_{i=1}^{m} F_{\rho}\left(\alpha_{i}, \beta_{i}, \gamma_{i}\right) .
\end{aligned}
$$

Denote for later use

$$
S_{\rho}(\vec{\beta}, \vec{\alpha}, \vec{\gamma})=\sum_{i=1}^{m} s\left(\beta_{i}, \alpha_{i} ; \frac{\gamma_{i}+\beta_{i} \rho}{\alpha_{i}},-\rho\right)
$$

and

$$
F_{\rho}(\vec{\alpha}, \vec{\beta}, \vec{\gamma})=\sum_{i=1}^{m} F_{\rho}\left(\alpha_{i}, \beta_{i}, \gamma_{i}\right)
$$

Remark 1.13. (a) We want to point out a delicate fact. Suppose that $N$ is a Seifert $\mathbb{Z}$-homology sphere equipped as usual with a Thurston geometry. The base of $N$ is an orbi-sphere $S$. Since $H^{1}(N) \cong H^{2}(N) \cong 0$ there is a unique spin and an unique $\operatorname{spin}^{c}$ structure and thus in this case all the spinor bundles $\mathbb{S}_{L}$ are isomorphic. However there is a plethora of Dirac operators and it is very easy to confuse them. We propose below an "accounting" method.

Denote by $\mathbb{S}_{0}$ the spinor bundle associated to the unique spin structure. There are two obvious Dirac operators on $\mathbb{S}_{0}$. One is $\mathfrak{D}_{0}$ obtained traditionally using the Levi-Civita connection on $T N$ and the other is $D_{0}$ obtained using the adiabatic connection. They are related by

$$
D_{0}=\mathfrak{D}_{0}+\frac{\ell}{2}, \quad \ell=\operatorname{deg} N
$$

By using different connections on $\operatorname{det} \mathbb{S}_{0} \cong \underline{\mathbb{C}}$ we can obtain many other.

Another fundamental pair of examples of Dirac can be obtained in a similar way. If we think of $\mathbb{S}_{c a n}$ defined in (1.7) then

$$
\operatorname{det} \mathbb{S}_{c a n} \cong \operatorname{det} \mathbb{S}_{0} \otimes \mathfrak{K}^{-1} \text {. }
$$

Using the connection $\nabla^{-c a n}$ on $\mathfrak{K}^{-1}$ defined as the Levi-Civita connection on $K_{S}^{-1}$ we obtain the operators $\mathfrak{D}_{c a n}$ and $D_{\text {can }}$.

Also, for "any" line bundle $L \rightarrow N$ we can regard

$$
\operatorname{det} \mathbb{S}_{L} \cong \operatorname{det} \mathbb{S}_{c a n} \otimes L^{2} \cong \mathfrak{K}^{-1} \otimes L^{2}
$$


Thus any connection $A$ on $L$ induces a connection $A^{\otimes 2}$ on $L^{2}$ and in this manner we obtained the operators $\mathfrak{D}_{A}$ and $D_{A}$ we have been studying so far. More accurately

$$
\mathfrak{D}_{A}=\mathfrak{D}_{0} \otimes\left(\nabla^{-c a n} \otimes A^{\otimes 2}\right), \quad D_{A}=D_{0} \otimes\left(\nabla^{-c a n} \otimes A^{\otimes 2}\right) .
$$

In particular, we have

$$
D_{0}=D_{c a n} \otimes \nabla^{c a n} .
$$

We have computed eta invariants for two types of Dirac operators.

- Pullback type

$$
D_{\text {can }} \otimes\left(\pi^{*} A\right)^{\otimes 2}
$$

where $A$ is a connection on a line $V$-bundle $L \rightarrow S$.

- Flat type

$$
D_{\text {can }} \otimes A^{\otimes 2}
$$

where $A$ is a connection on the unique line bundle over $N$ such that $\nabla^{-c a n} \otimes A^{\otimes 2}$ is flat. In this case there is a unique line bundle on $N$ with a unique (up to gauge equivalence) flat connection. The above operator is none other than $D_{0} ! ! !$

(b) We can say something about the eta invariant of $\mathfrak{D}_{0}$ as well. In our case the metric on $N$ is normalized so that the regular fibers have length $2 \pi$. We deduce as in $\S 2.3$ of [N2]

$$
\eta\left(D_{0}\right)-\eta\left(\mathfrak{D}_{0}\right)+\frac{\ell}{6}\left(\ell^{2}-\chi\right) \in 2 \mathbb{Z}
$$

where $\chi=-\operatorname{deg} K_{S}$. If as in [N1] and [N2] we deform the metric on $N$ along the fibers so that they have length $2 \pi r$ with $0<r \ll 1$ then we can be more precise. Denote by $D_{0}(r)$ and $\mathfrak{D}_{0}(r)$ the new Dirac operators defined in terms of the new metric. Then

$$
\eta\left(D_{0}(r)\right)=\eta\left(D_{0}\right)
$$

and the considerations of $\S 2.3$ in [N2] lead immediately to the equality

$$
\eta\left(D_{0}(r)\right)+2 h_{1 / 2}=\eta\left(\mathfrak{D}_{0}(r)\right)-\frac{\ell}{6}\left(\ell^{2} r^{4}-\chi r^{2}\right)+\left\{\begin{array}{rl}
4 h_{1 / 2} & , \quad \ell>0 \\
0, & \ell<0
\end{array} .\right.
$$


Above $h_{1 / 2}$ denotes the dimension of the space of holomorphic sections of a possible nonexistent holomorphic square root of $K_{S}$. When such a square root does not exist we set $h_{1 / 2}=0$. Note that even when such a square root exists one verifies immediately that $\operatorname{deg}\left|K_{S}^{1 / 2}\right|=-1$ so there cannot be any such holomorphic sections. We conclude

$$
\eta\left(\mathfrak{D}_{0}(r)\right)=\eta\left(D_{0}\right)+\frac{\ell}{6}\left(\ell^{2} r^{4}-\chi r^{2}\right) .
$$

The invariant $\eta\left(D_{0}\right)$ can be computed using (1.34) The eta invariant of the signature operator on $N$ equipped with such a metric was computed in [O] (watch out for the Seifert invariant conventions there) and it is

$$
\eta_{s i g n}^{r}=-\frac{2 \ell}{3}\left(\ell^{2} r^{4}-\chi r^{2}\right)+\frac{\ell}{3}-\operatorname{sign}(\ell)-4 S(\vec{\beta}, \vec{\alpha})
$$

where

$$
S(\vec{\beta}, \vec{\alpha}):=\sum_{i} s\left(\beta_{i}, \alpha_{i} ; 0,0\right)=S(\vec{\beta}, \vec{\alpha} ; \overrightarrow{0})
$$

with $S(\vec{\beta}, \vec{\alpha} ; \vec{\gamma})$ defined in (1.16). We deduce

$$
4 \eta\left(\mathfrak{D}_{0}(r)\right)+\eta_{s i g n}^{r}=4 \eta\left(D_{0}\right)+\frac{\ell}{3}-\operatorname{sign}(\ell)-4 S(\vec{\beta}, \vec{\alpha}) .
$$

This expression is independent $r$ ! The reason we considered it is because it appears in the definition of the invariant introduced by Froyshov in [Fr]. The above expression can be alternatively defined as

$$
\mathbf{F}(N):=c_{1}(L)^{2}-\sigma(V)-\operatorname{sind}_{\mathbf{C}} \hat{\mathfrak{D}}_{\mathbf{A}}
$$

where $V$ is a an oriented simply connected 4-manifold with $\partial V=N, \sigma(V)$ is the signature of $V, L$ is the determinant line bundle associated to a spin ${ }^{c}$ structure on $V$ and $\hat{\mathfrak{D}}_{A}$ is a Dirac operator of this $\operatorname{spin}^{c}$ structure extending the operator $\mathfrak{D}_{0}$. The above index refers to the Atiyah-Patodi-Singer index. Since $N$ is a $\mathbb{Z}$-homology sphere we deduce from Rohlin's theorem that $c_{1}(L)^{2}-\sigma(V) \in 8 \mathbb{Z}$. Hence

$$
4 \eta\left(D_{0}\right)+\frac{\ell}{3}-\operatorname{sign}(\ell)-4 S(\vec{\beta}, \vec{\alpha}) \in 8 \mathbb{Z} .
$$

This happens for every Seifert homology sphere $\Sigma(\vec{\alpha})$ (in the notations of $[\mathrm{JN}])$. 
Example 1.14. Let us consider in some detail the special case of the Poincaré homology sphere. It is the Brieskorn sphere $N=\Sigma(2,3,5)$. The rational degree of $N$ is $\ell=-1 / 30$. The Seifert invariants are described in [JN]. (Note they use different conventions for the Seifert invariants). They are

$$
\vec{\alpha}=(2,3,5), \vec{\beta}=(1,2,4) .
$$

The degree of the canonical class is

$$
\operatorname{deg} K_{S}=-2+1 / 2+2 / 3+4 / 5=-1 / 30 .
$$

The canonical representative of the unique line bundle on $N$ is the trivial line bundle on $S$ which has degree 0 and singularity data $\vec{\gamma}=(0,0,0)$. The invariant $\rho$ in this case is $1 / 2$. Using Proposition 1.10 we deduce

$$
4 \eta\left(D_{0}\right)=539 / 90 \text {. }
$$

A simple computation shows that in this case

$$
\frac{\ell}{3}-\operatorname{sign}(\ell)-4 S(\vec{\beta}, \vec{\alpha})=181 / 90 \text {. }
$$

This shows

$$
\mathbf{F}(\Sigma(2,3,5))=8 .
$$

agreeing with (1.38). The Froyshov invariant of the Poincaré homology sphere is also 8 . In $\S 3.2$ we will describe a general method of producing upper estimates for the Froyshov invariants which we believe are actually optimal.

\section{Finite energy Seiberg-Witten monopoles.}

Throughout this section, a hat over an object will signal (unless otherwise indicated) that it is a 4-dimensional geometric object.

For example, if $N$ is a 3-manifold then on the tube $\mathbb{R} \times N$ there exist two exterior derivatives: the 3-dimensional exterior derivative $d$ along the slices $\{t\} \times N$ and the 4-dimensional exterior derivative $\hat{d}$ so that $\hat{d}=d t \wedge \partial_{t}+d$. If $A(t)$ is a family of connection on some vector bundle $E \rightarrow N$ then we get a bundle $\hat{E} \rightarrow \mathbb{R} \times N$ and we can think of the path $A(t)$ as a connection $\hat{A}$ on $\hat{E}$. We will denote by $F_{A(t)}$ the curvature of $A(t)$ on the slice $\{t\} \times N$ while $\hat{F}_{\hat{A}}$ will denote the curvature of $\hat{A}$ on the tube. 


\subsection{The 4-dimensional Seiberg-Witten equations.}

Let $\hat{N}$ denote an oriented 4-manifold (not necessarily compact), equipped with a Riemann metric $\hat{g}$. Denote by $\hat{*}$ the Hodge star operator defined by the metric $\hat{g}$ and the orientation of $\hat{N}$. Fix a connection $\hat{\nabla}$ on $T \hat{N}$ compatible with $\hat{g} . \hat{\nabla}$ need not be the Levi-Civita connection.

Denote by $\operatorname{Spin}_{c}(\hat{N})$ the collection of isomorphism classes of $\operatorname{spin}^{c}$ structures on $\hat{N}$. For each $\hat{\sigma} \in \operatorname{Spin}^{c}(\hat{N})$ we denote by $\operatorname{det} \hat{\sigma}$ the associated line bundle and by $\mathbb{S}_{\hat{\sigma}}=\hat{\mathbb{S}}_{\hat{\sigma}}^{+} \oplus \hat{\mathbb{S}}_{\hat{\sigma}}^{-}$the associated bundle of spinors. Note that $\operatorname{det} \hat{\sigma} \cong \operatorname{det} \hat{\mathbb{S}}_{\hat{\sigma}}^{+}$.

Denote by $\mathfrak{A}_{\hat{\sigma}}$ the space of hermitian connections on $\mathbb{S}_{\sigma}$ compatible with both the $\mathbb{Z}_{2}$-grading and the fixed background connection $\hat{\nabla}$. More precisely, $A \in \mathfrak{A}_{\hat{\sigma}}(\hat{N})$ if for any $\alpha \in \Omega^{1}(N)$, any $X \in \operatorname{Vect}(N)$ and any $\hat{\psi} \in C^{\infty}\left(\mathbb{S}_{\sigma}\right)$ we have

$$
\nabla_{X}^{A}(\hat{\mathbf{c}}(\alpha) \hat{\psi})=\hat{\mathbf{c}}\left(\nabla_{X} \alpha\right) \hat{\psi}+\hat{\mathbf{c}}(\alpha) \nabla_{X}^{A} \hat{\psi}
$$

where

$$
\hat{\mathbf{c}}: T^{*} \hat{N} \rightarrow \operatorname{Hom}\left(\hat{\mathbb{S}}_{\hat{\sigma}}^{+}, \mathbb{S}_{\hat{\sigma}}^{-}\right)
$$

denotes the Clifford multiplication. Any connection on $\operatorname{det} \hat{\sigma}$ determines a connection in $\mathfrak{A}_{\hat{\sigma}}$ and moreover, once we fix a connection $A_{0} \in \mathfrak{A}_{\hat{\sigma}}(\hat{N})$, we can identify $\mathfrak{A}_{\hat{\sigma}}(\hat{N})$ with $\mathrm{i} \Omega^{1}(\hat{N})$. To any connection $\hat{A} \in \mathfrak{A}_{\hat{\sigma}}(\hat{N})$ we can associate the Dirac operator

$$
\hat{D}_{\hat{A}}: \Gamma\left(\hat{\mathbb{S}}_{\hat{\sigma}}^{+}\right) \rightarrow \Gamma\left(\hat{\mathbb{S}}_{\hat{\sigma}}^{-}\right)
$$

defined as the composition

$$
\Gamma\left(\hat{\mathbb{S}}_{\hat{\sigma}}\right) \stackrel{\hat{\nabla}^{\hat{A}}}{\rightarrow} \Gamma\left(T^{*} \hat{N} \otimes \hat{\mathbb{S}}_{\hat{\sigma}}^{+}\right) \stackrel{\hat{\mathbf{c}}}{\rightarrow} \Gamma\left(\hat{\mathbb{S}}_{\hat{\sigma}}^{-}\right) .
$$

There is a natural quadratic map

$$
q: \Gamma\left(\hat{\mathbb{S}}_{\hat{\sigma}}^{+}\right) \rightarrow \operatorname{End}\left(\hat{\mathbb{S}}_{\hat{\sigma}}^{+}\right), \quad \hat{\psi} \mapsto q(\hat{\psi}),
$$

defined by

$$
q(\hat{\psi}) \hat{\phi}=\langle\hat{\phi}, \hat{\psi}\rangle \hat{\psi}-\frac{1}{2}|\hat{\psi}|^{2} \hat{\phi}
$$

In terms of Dirac's bra-ket notation $\tau(\hat{\psi})$ can be alternatively described as

$$
q(\langle\hat{\psi}|)=|\hat{\psi}\rangle\langle\hat{\psi}|-\frac{1}{2}\langle\hat{\psi} \mid \hat{\psi}\rangle \text {. }
$$


Note that for each $\hat{\psi}$ the endomorphism $q(\hat{\psi})$ is symmetric and traceless. The quantization map from the exterior algebra to the Clifford algebra extends the Clifford multiplication to a map

$$
\hat{\mathbf{c}}: \Lambda^{*} T^{*} \hat{N} \rightarrow \operatorname{End}\left(\hat{\mathbb{S}}_{\hat{\sigma}}\right)
$$

This map has the property that $\hat{\mathbf{c}}(\omega)$ is a traceless, skew-symmetric endomorphism of $\hat{\mathbb{S}}_{\hat{\sigma}}^{+}$for any $\hat{g}$-self-dual real valued 2 -form $\omega$.

The Seiberg-Witten equations (associated to the spin $^{c}$ structure $\hat{\sigma}$ ) are equations for a pair $(\hat{\psi}, \hat{A})=\left(\right.$ spinor in $\mathbb{S}_{\hat{\sigma}}^{+}$, connection in $\left.\mathfrak{A}_{\hat{\sigma}}(\hat{N})\right)$. More precisely, they are

$$
(\widehat{S W})\left\{\begin{array}{rl}
\hat{D}_{\hat{A}} \hat{\psi} & =0 \\
\frac{1}{2} \hat{\mathbf{c}}\left(\hat{F}_{\hat{A}}^{+}\right) & =q(\hat{\psi})
\end{array} .\right.
$$

In the remaining part of this subsection we will make further additional assumptions on the geometry and the topology of $\hat{N}$ and explain how this affects the Seiberg-Witten equations.

More precisely, assume the manifold $\hat{N}$ can be decomposed as

$$
\hat{N}=\hat{N}_{0} \cup[0, \infty) \times N
$$

where $\hat{N}_{0}$ is a compact oriented 4-manifold with boundary $\partial \hat{N}_{0}=N$. We will denote by $t$ the (outgoing) longitudinal coordinate on the cylindrical part of $N$.

Fix a tubular neighborhood $(-1,0] \times N$ of $N$ in $\hat{N}_{0}$, a metric $g$ on $N$ and a connection $\nabla$ compatible with $g$, not necessarily the Levi-Civita connection of $g$. We assume that along the infinite cylinder $(-1, \infty) \times N$ the metric $\hat{g}$ is a product metric $\hat{g}=d t^{2}+g$. We fix a connection $\hat{\nabla}$ compatible with $\hat{g}$ such that along the above cylindrical end it has the form

$$
\hat{\nabla}=\partial_{\tau} \wedge d t+\nabla
$$

We denoted by $\partial_{\tau}$ the $\hat{g}$-gradient of $\tau$ where $\tau: \hat{N} \rightarrow[0, \infty)$ is a smooth function which coincides with the canonical projection $[0, \infty) \times N \rightarrow[0, \infty)$ on the infinite neck.

Note that the $\operatorname{spin}^{c}$ structure $\hat{\sigma}$ induces a $\operatorname{spin}^{c}$ structure $\sigma$ on $N=\partial \hat{N}_{0}$. Denote by $\mathbb{S}_{\sigma} \rightarrow N$ the associated bundle of spinors and by $\mathbf{c}: T^{*} N \rightarrow$ End $(N)$ the corresponding Clifford multiplication. As in the 4-dimensional case we can define $\mathfrak{A}_{\sigma}(N)$. 
Fix a reference connection $\hat{A}_{0} \in \mathfrak{A}_{\hat{\sigma}}(\hat{N})$ which along the neck is equal to a product connection $d t \otimes \partial_{t}+A_{0}, A_{0} \in \mathfrak{A}_{\sigma}(N)$. Now define the configuration space $\widehat{\mathfrak{C}}$ as the set of pairs $\left(\psi, \hat{A}_{0}+\mathbf{i} \hat{a}\right):=(\hat{\psi}, \hat{A})=$ (spinor, connection) such that $(\hat{\psi}, \mathbf{i} \hat{a}) \in L_{l o c}^{2,2}\left(\hat{\mathbb{S}} \oplus \mathbf{i} T^{*} \hat{N}\right)$ and

$$
\hat{\nabla}_{\partial \tau}^{\hat{A}} \hat{\psi} \oplus i_{\partial_{\tau}}\left(\mathbf{i} \hat{F}_{\hat{A}}\right) \in L^{2}\left(\hat{\mathbb{S}}_{\hat{\sigma}} \oplus \mathbf{i} \Lambda^{1} T^{*} \hat{N}\right) .
$$

We denoted by $i_{\partial_{\tau}}$ the contraction by $\partial_{\tau}$. For brevity, will denote the elements of $\widehat{\mathfrak{C}}$ by the generic symbol $\hat{\boldsymbol{C}}$.

Definition 2.1. (a) A finite energy solution of $\left(\widehat{S W}_{\omega}\right)$ is a solution $(\hat{\psi}, \hat{A})$ such that $\left(\hat{\psi}, \hat{A}-\hat{A}_{0}\right) \in \widehat{\mathfrak{C}}$.

(b) A Seiberg-Witten tunneling is a finite energy solution on $\hat{N}=\mathbb{R} \times N$.

There is an infinite dimensional group $\widehat{\mathfrak{G}}$ acting on the configuration space, more precisely

$$
\widehat{\mathfrak{G}}=\left\{\gamma \in \operatorname{Map}\left(\hat{N}, S^{1}\right) ; \gamma \in L_{l o c}^{3,2}\right\} .
$$

The group $\widehat{\mathfrak{G}}$ acts (on the right) on $\widehat{\mathfrak{C}}$ and transforms finite energy solutions to finite energy solutions. Define

$$
\widehat{\mathfrak{M}}:=\{(\hat{\psi}, \hat{A}) \text { finite energy solutions of } \widehat{S W}\} / \widehat{\mathfrak{G}} \text {. }
$$

In this section we want to analyze the the Fredholm properties of the deformation complex naturally associated to $\widehat{\mathfrak{M}}$ when $N$ is a Seifert fibration.

We conclude this subsection with a simple but crucial observation which reveals the dynamical feature of the Seiberg-Witten equations on cylinders.

Note that if we set $J=\hat{\mathbf{c}}(d \tau)$ then $J$ induces isomorphisms

$$
\left.\left.\hat{\mathbb{S}}_{\hat{\sigma}}^{+}\right|_{N} \cong \hat{\mathbb{S}}_{\hat{\sigma}}^{-}\right|_{N} \cong \mathbb{S}_{\sigma}
$$

and

$$
\mathbf{c}(\alpha)=J \hat{\mathbf{c}}(\alpha), \quad \forall \alpha \in \Omega^{1}(N) \hookrightarrow \Omega^{1}([0, \infty) \times N) .
$$

A connection $\hat{A} \in \mathfrak{A}_{\hat{\sigma}}(\hat{N})$ is said to be in a temporal gauge if $i_{\partial_{\tau}}\left(\hat{A}-\hat{A}_{0}\right)=0$ along the infinite neck $[0, \infty) \times N$.

Assume now that $(\hat{\psi}, \hat{A})$ is a finite energy solution of $(\widehat{S W})$ such that $\hat{A}$ is in a temporal gauge. Along the neck we can write

$$
\hat{\psi}=\psi(t), \quad \hat{A}=A_{0}+\mathbf{i} a(t)
$$


where $A_{0}=\left.\hat{A}_{0}\right|_{N}, \psi(t) \in \Gamma\left(\mathbb{S}_{\sigma}\right), a(t) \in \Omega^{1}(N), \forall t \geq 0$. Then (along the neck)

$$
\hat{F}_{\hat{A}}^{+}=\frac{1}{2}\left\{\left(F_{a}+* \mathbf{i} \dot{a}\right)+d t \wedge\left(\mathbf{i} \dot{a}+* F_{a}\right)\right\}
$$

where $A_{0}+a(t)$ is the connection on the line bundle $\operatorname{det} \sigma$ restricted to the slice $\{t\} \times N, F_{a}=F_{A_{0}+\mathbf{i} a}$ denotes is curvature and $*$ denotes the Hodge star operator on $N . A_{0}+\mathbf{i} a(t)$ induces a Dirac operator

$$
D_{a}=D_{a(t)}: \Gamma\left(\mathbb{S}_{\sigma}\right) \rightarrow \Gamma\left(\mathbb{S}_{\sigma}\right) .
$$

Using (2.1) and (2.2) we deduce that along the neck

$$
\hat{D}_{\hat{A}}=J\left(\partial_{t}-D_{a}\right)
$$

The equality (2.3) now implies

$$
\hat{\mathbf{c}}\left(\hat{F}_{\hat{A}}^{+}\right)=\mathbf{c}\left(* F_{a}+\mathbf{i} \dot{a}\right) .
$$

Consequently, along the neck, in a temporal gauge, the Seiberg-Witten equations can be rewritten as

$$
\left\{\begin{array}{rl}
\dot{\psi} & =D_{a} \psi \\
\mathbf{i c}(\dot{a}) & =q(\psi)-\frac{1}{2} \mathbf{c}\left(* F_{a}\right)
\end{array} .\right.
$$

The right-hand-side of (2.4) arises when one considers the three dimensional counterpart of the Seiberg-Witten equations.

\subsection{The 3-dimensional Seiberg-Witten equations.}

To formulate these equations we need to consider a new configuration space. Fix a connection $A_{0} \in \mathfrak{A}_{\sigma}(N)$ and define

$$
\mathfrak{C}=\left\{(\psi, A) ;\left(\psi,\left(A-A_{0}\right) \in L^{1,2}\left(\mathbb{S}_{\sigma} \oplus \mathbf{i} T^{*} N\right)\right\} .\right.
$$

For brevity, its elements will be denoted by the symbol $\mathrm{C}$ and we will often write $\mathbf{C}=(\psi, a)$ instead of $\left(\psi, A_{0}+\mathbf{i} a\right)$ whenever no confusion is possible. There is an energy functional $\mathfrak{E}: \mathfrak{C} \rightarrow \mathbb{R}$ defined by

$$
\mathfrak{E}(\psi, A)=\frac{\mathbf{i}}{4} \int_{N} a \wedge\left(F_{A_{0}}+F_{A}\right)+\frac{1}{2} \int_{N}\left\langle\psi, D_{A} \psi\right\rangle d v_{g} .
$$


The gauge group

$$
\mathfrak{G}=\left\{\gamma \in \operatorname{Map}\left(N, S^{1}\right) ; \gamma \in L^{2,2}\right\}
$$

acts on $\mathfrak{C}$ and moreover

$$
\mathfrak{E}\left(\gamma^{-1} \cdot(\psi, A)\right)-\mathfrak{E}(\psi, A)=-\int_{N} \gamma^{-1} d \gamma \wedge F_{A_{0}}=2 \pi \mathbf{i} \int_{N} \gamma^{-1} d \gamma \wedge c_{1}\left(A_{0}\right)
$$

where we denoted by $c_{1}\left(A_{0}\right)$ the 2 -from representing the first Chern class of $\operatorname{det} \sigma$ associated to $A_{0}$ via the Chern-Weil construction. The $L^{2}$-gradient of $\mathfrak{E}$ is (see $[\mathrm{N} 1]$ or $[\mathrm{MOY}]$ )

$$
\nabla \mathfrak{E}(\psi, A)=\left[\begin{array}{c}
D_{A} \psi \\
q(\psi)-\frac{1}{2} * F_{A}
\end{array}\right]
$$

where we tacitly identified $q(\psi)$ with a purely imaginary 1-form via the Clifford multiplication. The 3-dimensional Seiberg-Witten equations can now be described as

$$
\nabla \mathfrak{E}(\mathrm{C})=0 \Longleftrightarrow\left\{\begin{array}{rll}
D_{A} \psi & = & 0 \\
\mathbf{c}\left(* F_{A}\right) & = & q(\psi)
\end{array} .\right.
$$

We see that (2.4) can be rewritten as a gradient flow equation

$$
\dot{\mathrm{C}}=\nabla \mathfrak{E}(\mathrm{C}) .
$$

This last equation suggests that as $t \rightarrow \infty \mathrm{C}(t)$ converges to a critical point of $\mathfrak{E}$. Assuming the finite energy condition this can be proved for arbitrary $N$ using the techniques of [MMR]. However, unlike the Yang-Mills situation, the nature of critical points and the manner in which they are organized are less transparent in the Seiberg-Witten case. This is the reason why we will concentrate on a special case.

In remaining of the section $N$ will be assumed to be a Seifert fibration determined by the line $V$-bundle bundle $\mathbb{L}_{0}=L(\ell, \vec{\beta})$ of rational degree $\ell \neq 0$ over the $V$-surface $\Sigma(g, \vec{\alpha}), S^{1} \hookrightarrow N \stackrel{\pi}{\rightarrow}$ $\Sigma$ equipped with the metric described in $\S 1.2$ As background $g$ compatible connection on $N$ we choose the adiabatic connection $\nabla^{\infty}$.

Fix a line bundle $L \mapsto N$ which, as explained in $\S 1.2$, determines a spin ${ }^{c}$ structure with associated bundle of spinors $\mathbb{S}_{L}$. Using the decomposition $\mathbb{S}_{L} \cong\left(\mathfrak{K}^{-1} \otimes L\right) \oplus L$ we can represent any section $\psi$ of $\mathbb{S}_{L}$ as $\psi=\psi_{-} \oplus \psi_{+}$. 
In terms of a local, oriented orthonormal frame $\left(\zeta, \zeta_{1}, \zeta_{2}\right)$ with dual coframe $\left(\varphi, \varphi^{1}, \varphi^{2}\right)$ the Seiberg-Witten equations can be rephrased as (see [N1] or [MOY]) .

$$
\left\{\begin{array}{rr}
\mathbf{i} \nabla_{\zeta}^{A} \psi_{-}+\bar{\partial}_{A} \psi_{+} & =0 \\
\left(\bar{\partial}_{A}\right)^{*} \psi_{-}-\mathbf{i} \nabla_{\zeta} \psi_{+} & =0 \\
\frac{\mathbf{i}}{2}\left(\left|\psi_{+}\right|^{2}-\left|\psi_{-}\right|^{2}\right) & =F_{A}\left(\zeta_{1}, \zeta_{2}\right)-\frac{1}{2} F_{c a n}\left(\zeta_{1}, \zeta_{2}\right) \\
\mathbf{i} \psi_{-} \bar{\psi}_{+} & =\bar{\varepsilon} \otimes F_{A}\left(\zeta_{1}+\mathbf{i} \zeta_{2}, \zeta\right)
\end{array}\right.
$$

where $\varepsilon=2^{-1 / 2}\left(\varphi^{1}+\mathbf{i} \varphi^{2}\right)$, and $F_{c a n}$ denotes the pullback of the curvature of $K_{\Sigma}$ equipped with its Levi-Civita connection. Set

$$
\mathfrak{C}^{*}=\{(\psi, A) \in \mathfrak{C} ; \psi \not \equiv 0\} .
$$

The configurations in $\mathfrak{C}^{*}$ are called irreducible. As in $[\mathrm{M}]$ one can show that $\mathfrak{B}:=\mathfrak{C} / \mathfrak{G}$ is a metric space and, moreover, $\mathfrak{B}^{*}=\mathfrak{C}^{*} / \mathfrak{G}$ is a Banach manifold. This is proved using the existence of local slices for the $\mathfrak{G}$-action exactly as in the Yang-Mills case. For every configuration $C \in \mathfrak{C}$ we will denote by $[\mathcal{C}]$ its image in $\mathfrak{B}$.

The solutions of (2.7) are explicitly described in [MOY]. Here are the relevant facts.

Fact 1. If $c_{1}(L)$ is not torsion then (2.7) has no solutions.

Assume now that $c_{1}(L)=\kappa \in \operatorname{Pic}^{t}(\Sigma) / \mathbb{Z}\left[\mathbb{L}_{0}\right]$ and define

$$
\begin{gathered}
\tilde{R}_{\kappa}=\left\{E \in \operatorname{Pic}^{t}(\Sigma) ; 0<\left|\operatorname{deg} E-\frac{1}{2} \operatorname{deg} K_{\Sigma}\right| \leq \frac{1}{2} \operatorname{deg} K_{\Sigma},\right. \\
\left.E \equiv \kappa \bmod \mathbb{Z}\left[\mathbb{L}_{0}\right]\right\} .
\end{gathered}
$$

For each $E \in \tilde{R}_{\kappa}$ set $\nu(E)=\operatorname{deg} E-\frac{1}{2} \operatorname{deg} K_{\Sigma}$. Note that since $\ell \neq 0$ the map $\nu: \tilde{R}_{\kappa} \rightarrow \mathbb{Q}$ is injective. We will often identify $\tilde{R}_{\kappa}$ with its image in $\mathbb{Q}$ via $\nu$. Now set

$$
\begin{aligned}
& R_{\kappa}^{-}=\left\{E \in \tilde{R}_{\kappa} ; \nu(E)<0, \operatorname{deg}|E| \geq 0\right\} \\
& R_{\kappa}^{+}=\left\{E \in \tilde{R}_{\kappa} ; \nu(E)>0, \operatorname{deg}\left|K_{\Sigma}-E\right| \geq 0\right\}
\end{aligned}
$$


and

$$
R_{\kappa}=R_{\kappa}^{-} \cup R_{\kappa}^{+}
$$

Fact 2. Any irreducible solution $(\phi, A)$ of (2.7) is gauge equivalent to the pullback of a pair $(\tilde{\phi}, B)$ where $B$ is a connection in a $V$-line bundle $E \rightarrow \Sigma$ in $R_{\kappa}$ such that $\tilde{\phi}=\tilde{\phi}_{-} \oplus \tilde{\phi}_{-}$is a section of $C^{\infty}\left(K^{-1} \otimes E \oplus E\right)$. The connection $B$ defines holomorphic structures in $K^{-1} \otimes E$ and $E$. $\tilde{\phi}_{-}$is an antiholomorphic section of $K^{-1} \otimes E$ while $\tilde{\phi}_{+}$is a holomorphic section of $E$. Moreover, exactly one of $\tilde{\phi}_{-}$or $\tilde{\phi}_{+}$is zero according to the identity:

$$
\frac{1}{4 \pi} \int_{\Sigma}\left(\left|\tilde{\phi}_{-}\right|^{2}-\left|\tilde{\phi}_{+}\right|^{2}\right) d v=\nu(E) \neq 0 .
$$

Thus $\tilde{\phi}_{+}=0$ iff $\nu(E)>0$ and $\tilde{\phi}_{-}=0$ iff $\nu(E)<0$. Pairs $\left(\tilde{\phi}_{-} \oplus \tilde{\phi}_{+}, B\right)$ as above are known as vortex pairs on $\Sigma$ corresponding to the $V$-line bundle $E$. If $\nu(E)<0$ we say we have a holomorphic vortex on $E$ while if $\nu(E)>0$ we say we have an antiholomorphic vortex on $E$.

The irreducible part $(\bmod \mathfrak{G})$, denoted by $\mathfrak{M}^{*}$ consists of $\# R_{\kappa}$ components

$$
\mathfrak{M}^{*}=\bigcup_{E \in R_{\kappa}} \mathfrak{M}_{\kappa, E}=\bigcup_{n \in \nu\left(R_{\kappa}\right)} \mathfrak{M}_{\kappa, n}
$$

The component $\mathfrak{M}_{n}=\mathfrak{M}_{\kappa, n}$ corresponding to a choice $\nu(E)=n<0$ is diffeomorphic to a symmetric product of $\operatorname{deg}|E|$ copies of $\Sigma$. If $n=\nu(E)>0$ the moduli space is isomorphic to a symmetric product of $\operatorname{deg}|K-E|$ copies of $\Sigma$. Each component is Bott nondegenerate as a critical set.

Fact 3. The reducible solutions consist of pairs $(0, A)$ where $A$ is a connection on $E$ satisfying (1.23). Modulo $\mathfrak{G}$ they form a space $\mathfrak{M}_{\kappa}^{0}$ homeomorphic to the jacobian $J(|\Sigma|)$ which is a $2 g$-dimensional torus. If there exist degenerate reducible (i.e. $\operatorname{ker} D_{A} \neq 0$ ) then invariant $\rho$ of $L \rightarrow N$ must be zero. In this case the degenerate solutions can be identified with the Brill-Noether locus consisting of all holomorphic line $V$-bundles of degree $\operatorname{deg} K_{\Sigma} / 2$ which admit nontrivial holomorphic sections.

Fact 4. If in the definition of $\mathbb{E}$ we fix the reference connection such that $\mathfrak{E} \equiv 0$ on the reducible part of $\mathfrak{M}$ then the energy along the component 
$R_{\kappa, E}$ can be expressed as const. $\nu(E)^{2} / \ell$, where const is a certain positive universal constant irrelevant in the sequel.

Associated to each component $\mathfrak{M}$ there is a deformation theory which we now proceed to describe. We will concentrate only on the irreducible part $\mathfrak{C}^{*}$. Since $c_{1}(L)$ is torsion the energy functional $\mathfrak{E}$ is gauge invariant and thus it descends to a well defined functional

$$
\underline{E}: \mathfrak{B}^{*} \rightarrow \mathbb{R} \text {. }
$$

The group $\mathfrak{G}$ is a Hilbert-Lie group and its Lie algebra can be identified with the space $\mathfrak{g}:=L^{2,2}(N, \mathfrak{i} \mathbb{R})$. The exponential map has the form

$$
\mathfrak{g} \ni \mathbf{i} f \mapsto\left(\exp (\mathbf{i} f): N \rightarrow S^{1}\right) .
$$

The tangent space to the orbit $\mathcal{O}_{\phi, A}$ through $\mathrm{C}=(\phi, A)$ of the right action of $\mathfrak{G}$ is the range of the infinitesimal action operator

$$
\mathfrak{L}=\mathfrak{L}_{\mathrm{C}}: \mathfrak{g} \rightarrow \mathfrak{X}:=L^{1,2}\left(\mathbb{S}_{L}\right) \oplus L^{1,2}\left(\mathbf{i} T^{*} N\right), \quad \text { if } \mapsto-\mathbf{i} f \oplus \mathbf{i} d f .
$$

The tangent space to $\mathfrak{B}^{*}$ at $[C]$ can be identified with the orthogonal complement to the tangent space to the orbit $\mathcal{O}_{\mathrm{C}}$ and ultimately with the kernel of $\mathfrak{L}_{\mathcal{C}}^{*}$, the adjoint of $\mathfrak{L}_{\mathrm{C}}$. An integration by parts shows

$$
\mathfrak{L}^{*}(\dot{\psi} \oplus \mathbf{i} \dot{a})=-\mathbf{i} d^{*} a+\mathbf{i} \mathfrak{I} m\langle\phi, \dot{\psi}\rangle, \quad \forall \dot{\psi} \oplus \mathbf{i} \dot{a} \in \mathfrak{X} .
$$

We can use the affine structure of $\mathfrak{C}$ to linearize $\nabla \mathfrak{E}$ at a given configuration $\mathrm{C}=(\phi, A)$ and we obtain the unrestricted hessian at $\mathrm{C}$

$$
\mathfrak{H C}\left[\begin{array}{c}
\dot{\psi} \\
\mathbf{i} \dot{a}
\end{array}\right]=\left.\frac{d}{d t}\right|_{t=0} \nabla \mathfrak{E}(\psi+t \dot{\psi}, A+t \mathbf{i} \dot{a})=\left[\begin{array}{c}
D_{A} \dot{\psi}+\mathbf{c}(\mathbf{i} \dot{a}) \phi \\
-\mathbf{i} * d \dot{a}+\dot{q}(\phi, \dot{\psi})
\end{array}\right] .
$$

The term $\dot{q}(\phi, \dot{\psi})$ is formally defined by the equality

$$
\dot{q}(\phi, \dot{\psi}):=\left.\frac{d}{d t}\right|_{t=0} q(\phi+t \dot{\psi})
$$

where we regard $q$ as a quadratic map $q: \mathbb{S}_{L} \rightarrow \mathbf{i} T^{*} N$.

The stabilized hessian of $\mathfrak{E}$ at $\mathrm{C}=(\phi, A)$ is the unbounded operator on $L^{2}\left(\mathbb{S}_{L} \oplus \mathbf{i}\left(\Lambda^{1} \oplus \Lambda^{0}\right) T^{*} N\right)$ defined by

$$
\begin{aligned}
\tilde{\mathfrak{H}}_{C}\left[\begin{array}{c}
\dot{\psi} \oplus \mathbf{i} \dot{a} \\
\mathbf{i} f
\end{array}\right] & :=\left[\begin{array}{cc}
\mathfrak{H} & \mathfrak{L} \\
\mathfrak{L}^{*} & 0
\end{array}\right]\left[\begin{array}{c}
\dot{\psi} \oplus \mathbf{i} \dot{a} \\
\mathbf{i} f
\end{array}\right] \\
& =\left[\begin{array}{lll}
D_{A} \dot{\psi} & & \mathbf{c}(\mathbf{i} \dot{a}) \phi-\mathbf{i} f \phi \\
& -\mathbf{i} * d \dot{a}+\mathbf{i} d f & +\dot{q}(\phi, \dot{\psi}) \\
& \mathbf{i} d^{*} \dot{a} & +\mathbf{i} \mathfrak{I} m\langle\phi, \dot{\psi}\rangle
\end{array}\right] .
\end{aligned}
$$


In [MOY] it is shown that if $[\mathrm{C}] \in \mathfrak{M}_{\kappa, n}$ then the kernel of the stabilized hessian $\tilde{H}_{\mathrm{C}}$ is naturally isomorphic to the tangent space $T_{[\mathrm{C}]} \mathfrak{M}_{\kappa, n}$. Now define

$$
\tilde{\mathfrak{H}}_{0}\left[\begin{array}{c}
\dot{\psi} \\
\mathbf{i} \dot{a} \\
\mathbf{i} f
\end{array}\right]=\left[\begin{array}{rrr}
D_{A} & 0 & 0 \\
0 & -* d & d \\
0 & d^{*} & 0
\end{array}\right]\left[\begin{array}{c}
\dot{\psi} \\
\mathbf{i} \dot{a} \\
\mathbf{i} f
\end{array}\right]
$$

and $\mathcal{P}=\mathcal{P}_{\phi}$ by

$$
\mathcal{P}_{\phi}\left[\begin{array}{c}
\dot{\psi} \\
\mathbf{i} \dot{a} \\
\mathbf{i} f
\end{array}\right]=\left[\begin{array}{c}
\mathbf{c}(\mathbf{i} \dot{a}) \phi-\mathbf{i} f \phi \\
\dot{q}(\phi, \dot{\psi}) \\
\mathrm{i} \Im m\langle\phi, \dot{\psi}\rangle
\end{array}\right]
$$

Note that $\tilde{\mathfrak{H}}_{0}=\tilde{\mathfrak{H}}_{0}(\mathrm{C})$ is an elliptic selfadjoint operator for any $\mathrm{C} \in \mathfrak{C}$ and $\tilde{\mathfrak{H}}_{\mathrm{C}}=\tilde{\mathfrak{H}}_{0}+\mathcal{P}_{\phi}$. For every $\mathrm{C} \in \mathfrak{C}$ define $S F(\mathrm{C}) \in \mathbb{Z}$ as the spectral flow of the path $\tilde{\mathfrak{H}}_{0}(\mathrm{C})+t \mathcal{P}_{\phi}, t \in[0,1]$.

This spectral flow can be computed exactly as in Sec. $\S 3.3$ of [N2]. More precisely, suppose $\mathrm{C} \in \mathfrak{M}_{\kappa, n}$. Thus $\mathrm{C}$ is the pull back of a vortex pair $\left(\phi_{-} \oplus \phi_{+}, B\right)$ corresponding to a $V$-line bundle $L_{\Sigma} \rightarrow \Sigma$ in $R_{\kappa}$ with $\nu\left(L_{\Sigma}\right)=$ n. $B$ induces a holomorphic structure on this bundle and we denote by $\mathbb{L}_{C}$ the resulting holomorphic $V$-line bundle. If we set $\varepsilon(\ell)=(1+\operatorname{sign}(\ell)) / 2$ we then get

$$
S F(\mathrm{C})=\left\{\begin{array}{rrr}
-1-\varepsilon(\ell)-2 h_{0}\left(K_{\Sigma}-\mathbb{L}_{\mathrm{C}}\right) & \text { if } & n<0 \\
-1-\varepsilon(\ell)-2 h_{0}\left(\mathbb{L}_{\mathrm{C}}\right) & \text { if } & n>0
\end{array} .\right.
$$

\subsection{Virtual dimensions.}

In this subsection we will compute virtual dimensions of finite energy moduli spaces. We will rely heavily on the techniques of [MMR].

Consider a 4-manifold $\hat{N}$ with a cylindrical end isometric to $[0, \infty) \times N$ where $N$ is disjoint union of Seifert manifolds $\left\{N_{j} ; j=1, \ldots, n\right\}$ of rational degrees $\ell_{j}$, singularities $\left(\vec{\alpha}_{j}, \vec{\beta}_{j}\right)$ over Riemann surfaces $\Sigma_{j}$ of genera $g_{j}$. To ease the notational burden we will consider only the case $n=1$ (i.e. the boundary is connected). Thus we will drop the index $j$ in the notation of the above objects. Assume $N$ has $m$ singular fibers with with singularities $(\vec{\alpha}, \vec{\beta})$.

Fix a $\operatorname{spin}^{c}$ structure $\hat{\sigma}$ on $\hat{N}$. This induces a $\operatorname{spin}^{c}$ structure $\sigma$ on $N$ determined by a line bundle $L$. The metric and compatible connections on 
the end of $\hat{N}$ are prescribed as indicated in $\S 2.2$. This means that as background connection on $N$ we use the adiabatic connection $\nabla^{\infty}$. Consider a finite energy, irreducible, solution $\hat{C}=(\hat{\phi}, \hat{A})$ of the Seiberg-Witten equations associated to the structure $\hat{\sigma}$ and we assume that along the neck it is in temporal gauge

$$
\hat{\mathrm{C}}=\{t \mapsto \mathrm{C}(t)=(\phi(t), A(t))\} .
$$

The techniques of [MMR] work with no essential changes in the SeibergWitten context and show that $[C(t)]$ converges to $\left[C_{\infty}\right] \in \mathfrak{M}_{L}$, where by $\mathfrak{M}_{L}$ we denoted the Seiberg-Witten moduli space determined by the $\operatorname{spin}^{c}$ structure $\sigma$ on $N$. The first conclusion we draw from this fact is that $L$ must be a pulled back line bundle e since otherwise $\mathfrak{M}_{L}=\emptyset$. Suppose this is indeed the case and set $\kappa=c_{1}(L)$.

The moduli space $\mathfrak{M}_{\kappa}$ is a disjoint union

$$
\mathfrak{M}_{\kappa}=\bigcup_{n} \mathfrak{M}_{\kappa, n} .
$$

Assume first that the configuration $C_{\infty}$ is irreducible

$$
\mathrm{C}_{\infty} \in \mathfrak{M}_{\kappa, n}(\sigma) .
$$

Again, to ease the notation we set $\mathrm{C}=\mathrm{C}_{\infty}$. For simplicity assume $n<0$ so that is $\mathrm{C}=\left(\phi_{-} \oplus, \phi_{+}, A\right)$ then $\phi_{-}=\equiv 0$. The configuration $\mathrm{C}$ is pulled back from a vortex pair $(\tilde{\phi}, B)$ corresponding to a $V$ line bundle $L_{\Sigma}=L(c, \vec{\gamma}) \in$ $R_{\kappa}$.

We are interested in describing a neighborhood of $\hat{C}$ in the moduli space of finite energy solutions and we will begin as in [MMR] by studying a simpler problem.

Define $\widehat{\mathfrak{M}}([\mathrm{C}])$ as the moduli space of finite energy solutions with asymptotic limit $\left[C_{\infty}\right]$. We want to understand the structure of a small neighborhood of $\hat{C} \in \widehat{\mathfrak{M}}([C])$. More precisely, we would like to compute the virtual dimension of such a neighborhood. This is achieved using Kuranishi's deformation picture of the moduli space which requires a suitable functional framework.

By Fact 2, all the irreducible monopoles are Bott nondegenerate and the convergence to any irreducible asymptotic limit is exponential. This is a very fortunate situation since we can completely avoid the introduction of the thickened moduli spaces and instead we can use the very convenient weighted Sobolev spaces $L_{w}^{k, p}$ where $w$ is a very small positive number (see 
also [MMS]) The resulting deformation complex can be described exactly as in Chap. 8 of [MMR] and is

$$
0 \rightarrow X_{0} \stackrel{\hat{\mathfrak{L}}_{\hat{c}}}{\longrightarrow} X_{1} \stackrel{s w}{\longrightarrow} X_{2} \rightarrow 0 .
$$

where $X_{0}$ is the Lie algebra of the group of gauge transformations on $\hat{N}$ exponentially converging to 1 along the neck

$$
X_{0}=L_{w}^{3,2}\left(\mathbf{i} \Lambda^{0} T^{*} \hat{N}\right) .
$$

$X_{1}$ is the tangent space to the space of configurations of the 4-dimensional equations

$$
X_{1}=L_{w}^{2,2}\left(\hat{\mathbb{S}}_{\hat{\sigma}}^{+} \oplus \mathbf{i} \Lambda^{1} T^{*} \hat{N}\right) .
$$

$X_{2}$ is the space of "obstructions"

$$
X_{2}=L_{w}^{1,2}\left(\hat{\mathbb{S}}_{\hat{\sigma}}^{-} \oplus \mathbf{i} \Lambda_{+}^{2} T^{*} \hat{N}\right) .
$$

$\hat{\mathfrak{L}}=\hat{\mathfrak{L}}_{\hat{\mathrm{C}}}$ is the infinitesimal gauge group action at $\hat{\mathrm{C}}$ and $\underline{s w}$ is the linearization at $\hat{C}$ of the $S W$-equations on $\hat{N}$.

We can now form the operator

$$
\hat{\mathcal{O}}_{w}: X_{1} \rightarrow X_{2} \oplus X_{0}, \quad \hat{\mathcal{O}}_{w}=\underline{s w} \oplus \hat{\mathfrak{L}}^{* w}
$$

where ${ }^{*}{ }^{w}$ denotes the $L_{w}^{2}$-adjoint of $\hat{\mathfrak{L}}$. This is an elliptic operator and a computation $\grave{a}$ la [MMR] (Chap. 8) shows that along the neck it exponentially approaches

$$
\hat{\mathcal{O}}_{w}=\text { something } \times\left(\nabla_{t}-\mathcal{O}_{w}\right)
$$

where

$$
\mathcal{O}_{w}\left[\begin{array}{c}
\dot{\psi} \oplus \dot{\mathbf{i} a} \\
\mathbf{i} f
\end{array}\right]=\left[\begin{array}{lll}
D_{A} \dot{\psi} & & +\mathbf{c}(\mathbf{i} \dot{a}) \phi-\mathbf{i} f \phi \\
& -\mathbf{i} * d \dot{a}+\mathbf{i} d f & +\dot{q}(\phi, \dot{\psi}) \\
& \mathbf{i} d^{*} \dot{a}-2 w \mathbf{i} f & +\mathbf{i} \mathfrak{I} m\langle\phi, \dot{\psi}\rangle
\end{array}\right]
$$

and $[\phi, A]=[\mathrm{C}]$. Note that $\tilde{\mathfrak{H}}_{1}\left(\mathrm{C}_{\infty}\right)=\left.\mathcal{O}_{w}\right|_{w=0} . \hat{\mathcal{O}}_{w}$ is a Fredholm operator and its index (over $\mathbb{R}$ ) is equal to the virtual dimension of a small neighborhood of $[\hat{\mathrm{C}}]$ in $\widehat{\mathfrak{M}}([\mathrm{C}])$. We conclude as in $\S 3.4$ of [N2] that the index of $\hat{\mathcal{O}}_{w}$ is equal to the Atiyah-Patodi-Singer index $\left(\operatorname{ind}_{A P S}\right)$ of $\hat{\mathcal{O}}_{w}$. 
Denote by $\mathcal{A}$ the anti-selfduality operator on $\hat{N}$

$$
\mathcal{A}=d_{+} \oplus d^{*}: \mathbf{i} \Omega^{1}(\hat{N}) \rightarrow \mathbf{i} \Omega^{2}(\hat{N}) \oplus \mathbf{i} \Omega^{0}(\hat{N}) .
$$

Using the connection $\hat{A}$ and the spin $^{c}$ structure $\hat{\sigma}$ we can form the Dirac operator

$$
\hat{D}_{\hat{A}}: \Gamma\left(\hat{\mathbb{S}}_{\hat{\sigma}}^{+}\right) \rightarrow \Gamma\left(\hat{\mathbb{S}}_{\hat{\sigma}}^{-}\right)
$$

Along the neck the direct sum $\mathcal{N}_{\hat{\mathrm{C}}}=\hat{D}_{\hat{A}} \oplus \mathcal{A}$ has the $A P S$ form

$$
\mathcal{N}=\text { something } \times\left(\nabla_{t}-\tilde{\mathfrak{H}}_{0}\left(\mathrm{C}_{\infty}\right)\right) .
$$

Using the excision formula in [N2] we deduce

$$
\operatorname{ind}_{A P S}\left(\hat{\mathcal{O}}_{w}\right)=\operatorname{ind}_{A P S}(\mathcal{N})-S F\left(\tilde{\mathfrak{H}}_{0} \rightarrow \tilde{\mathfrak{H}}_{1}\right)-S F\left(\tilde{\mathfrak{H}}_{1} \rightarrow \mathcal{O}_{w}\right)
$$

where $S F(A \rightarrow B)$ denotes the spectral flow $S F(A+t(B-A), t \in[0,1])$. All the indices and the spectral flows above are real quantities.

We now proceed to determine the three terms in the right-hand side of the above formula.

Arguing as in the Appendix D of [N2] we conclude that the third term above vanishes. The second spectral flow can be rewritten as

$$
S F\left(\tilde{\mathfrak{H}}_{0} \rightarrow \tilde{\mathfrak{H}}_{1}\right)=S F([\mathrm{C}]) .
$$

We denote by $\rho_{\text {asd }}$ (resp. $\rho_{\text {dir }}$ ) the index densities of $\mathcal{A}$ (resp. $\hat{D}_{\hat{A}}$ )

$$
\rho_{\text {asd }}=-\frac{1}{2}(\mathbf{e}(\hat{N})+\mathbf{L}(\hat{N})) .
$$

where $\mathbf{e}(\hat{N})$ and $\mathbf{L}(\hat{N})$ denote respectively the Euler and the $L$-genus forms on $\hat{N}$ constructed using the Levi-Civita connection. Also

$$
\rho_{\text {dir }}=2 \hat{\mathbf{A}}\left(\hat{\nabla}^{0}\right) \wedge \exp \left(\frac{1}{2} c_{1}(\operatorname{det} \hat{\sigma})\right)
$$

where on $\operatorname{det} \hat{\sigma}$ we used the connection induced by $\hat{A}$. The factor 2 appears since we are interested in the real index of $\hat{D}$. The $\hat{\mathbf{A}}$-genus form is computed using the metric compatible connection $\hat{\nabla}^{0}$ which along the neck has the product form $d t \otimes \partial_{t}+\nabla^{0}$. We denote by $c(\hat{A})$ the form

$$
\frac{\mathbf{i}}{2 \pi} \hat{F}_{\hat{A}}
$$


on $\hat{N}$ where $\hat{A}$ denotes the induced connection on $\operatorname{det} \hat{\sigma}$.

On a 4-manifold the above equality has a simpler form

$$
\rho_{\text {dir }}=\frac{1}{4}\left(c(\hat{A})^{2}-\mathbf{L}\left(\hat{\nabla}^{0}\right)\right) .
$$

The $\xi$ invariant of $\tilde{\mathfrak{H}}_{0}$ is the sum $\xi\left(\left.\mathcal{A}\right|_{N}\right)+2 \xi\left(D_{A}\right)$ (the factor 2 is present for reality reasons).

$$
\xi\left(\tilde{\mathfrak{H}}_{0}\right)=\frac{1}{2}\left(\operatorname{dim}_{\mathbf{R}} \operatorname{ker} \tilde{\mathfrak{H}}_{0}-\eta_{s i g n}+2 \eta\left(D_{A}\right)\right)
$$

where $\eta_{s i g n}$ denotes the eta invariant of the odd signature operator. We deduce

$$
\begin{aligned}
\operatorname{ind}_{A P S}(\mathcal{N})= & \int_{N}\left(\rho_{\text {asd }}+\rho_{\text {dir }}\right)-\xi\left(\tilde{\mathfrak{H}}_{0}\right) \\
= & -\frac{1}{2} \int_{\hat{N}} \mathbf{e}-\frac{1}{2}\left(\int_{\hat{N}} \mathbf{L}-\eta_{s i g n}\right)+\frac{1}{4} \int_{\hat{N}}\left(c^{2}(\hat{A})-\mathbf{L}\left(\hat{\nabla}^{0}\right)\right) \\
& -\frac{1}{2} \operatorname{dim}_{\mathbf{R}} \operatorname{ker} \tilde{\mathfrak{H}}_{0}-\eta\left(D_{A}\right) .
\end{aligned}
$$

In [N1] and [MOY] it is shown that the kernel of $\tilde{\mathfrak{H}}_{0}$ is isomorphic to

$$
H^{0}(N, \mathbb{R}) \oplus H^{1}(N, \mathbb{R}) \oplus \operatorname{ker} D_{A}, \operatorname{ker} D_{A} \cong H^{0}\left(\mathbb{L}_{\mathrm{C}}\right)+H^{0}\left(K_{\Sigma}-\mathbb{L}_{\mathrm{C}}\right) .
$$

Using the equality (1.15) we deduce

$$
\begin{aligned}
\operatorname{ind}_{A P S}\left(\hat{\mathcal{O}}_{w}\right)= & -(\chi(\hat{N})+\operatorname{sign}(\hat{N})) / 2+\frac{1}{4} \int_{\hat{N}}\left(c^{2}(\hat{A})-\mathbf{L}\left(\hat{\nabla}^{0}\right)\right) \\
& -\operatorname{dim}_{\mathbf{C}} \operatorname{ker} D_{A}-\frac{1}{2}(2 g+1)-\frac{\ell}{6}+2 S(\vec{\beta}, \vec{\alpha} ; \vec{\gamma})+d(\vec{\beta}, \vec{\alpha} ; \vec{\gamma}) .
\end{aligned}
$$

Using (2.10) and (2.11) we deduce

$$
\begin{aligned}
& \text { ind }\left(\hat{\mathcal{O}}_{w}\right)=-\frac{1}{2}(\chi(\hat{N})+\operatorname{sign}(\hat{N}))+\frac{1}{4} \int_{\hat{N}}\left(c^{2}(\hat{A})-\mathbf{L}\left(\hat{\nabla}^{0}\right)\right) \\
& -\left(\operatorname{dim}_{\mathbf{C}} \operatorname{ker} D_{A}+S F([\mathrm{C}])\right)-\frac{1}{2}(1+2 g)-\frac{\ell}{6}+S(\vec{\beta}, \vec{\alpha} ; \vec{\gamma})+d(\vec{\beta}, \vec{\alpha} ; \vec{\gamma}) .
\end{aligned}
$$

This formula can be further simplified using (2.8) (with $n<0$ ). We have

$$
\begin{gathered}
\operatorname{dim}_{\mathrm{C}} \operatorname{ker} D_{A}+S F([\mathrm{C}])=h_{0}\left(\mathbb{L}_{\mathrm{C}}\right)-h_{0}\left(K_{\Sigma}-\mathbb{L}_{\mathrm{C}}\right)-1-\varepsilon(\ell) \\
(\text { Riemann-Roch-Kawasaki) } \\
=\operatorname{deg}\left|\mathbb{L}_{\mathrm{C}}\right|-g-\varepsilon(\ell) .
\end{gathered}
$$


We can replace the integral of $\mathbf{L}\left(\hat{\nabla}^{0}\right)$ with the integral of $\mathbf{L}(\hat{N})$ plus a correction term given by a transgression. This correction term can be computed exactly as in the proof of the second transgression formula of [N2] and we get (assuming the radius of a regular fiber of $N$ is 1 )

$$
\int_{\hat{N}} \mathbf{L}\left(\hat{\nabla}^{0}\right)-\int_{\hat{N}} \mathbf{L}(\hat{N})=\frac{2 \ell}{3}\left(\ell^{2}-\chi\right)
$$

where $\chi=-\operatorname{deg} K_{\Sigma}$. The signature eta invariant of $N$ was computed in [O] and is given by

$$
\eta_{\text {sign }}=-\operatorname{sign}(\ell)-\frac{2 \ell}{3}\left(\ell^{2}-\chi\right)+\frac{\ell}{3}-4 S(\vec{\beta}, \vec{\alpha})
$$

where, as in Remark 1.13

$$
S(\vec{\beta}, \vec{\alpha})=\sum_{i=1}^{m} s\left(\beta_{i}, \gamma_{i} ; 0,0\right) .
$$

We deduce from (2.13)

$$
\int_{\hat{N}} \mathbf{L}\left(\hat{\nabla}^{0}\right)+\eta_{s i g n}-\int_{\hat{N}} \mathbf{L}(\hat{N})=\ell / 3-\operatorname{sign}(\ell)-4 S(\vec{\beta}, \vec{\alpha}) .
$$

The term

$$
\eta_{s i g n}-\int_{\hat{N}} \mathbf{L}(\hat{N})
$$

is equal to $-\operatorname{sign}(\hat{N})$ so that we deduce

$$
\int_{\hat{N}} \mathbf{L}\left(\hat{\nabla}^{0}\right)=\operatorname{sign}(\hat{N})+\frac{\ell}{3}-\operatorname{sign}(\ell)-4 S(\vec{\beta}, \vec{\alpha})
$$

If we use this equality in (2.12) we deduce

$$
\begin{aligned}
\operatorname{ind}_{A P S}\left(\hat{\mathcal{O}}_{w}\right)= & \frac{1}{4} \int_{\hat{N}} c^{2}(\hat{A})-\frac{1}{4}(2 \chi(\hat{N})+3 \operatorname{sign}(\hat{N})) \\
& +\left(g+\varepsilon(\ell)-\operatorname{deg}\left|\mathbb{L}_{C}\right|\right)-\frac{1}{2}(2 g+1)-\frac{1}{4}(\ell / 3-\operatorname{sign}(\ell)) \\
& -\frac{\ell}{6}+2 S(\vec{\beta}, \vec{\alpha}, \vec{\gamma})+d(\vec{\beta}, \vec{\alpha}, \vec{\gamma})+S(\vec{\beta}, \vec{\alpha}) . \\
= & d(\hat{\sigma})-\frac{1}{2}+\varepsilon(\ell)+\frac{\operatorname{sign}(\ell)}{4}-\frac{\ell}{4}-\operatorname{deg}\left|\mathbb{L}_{C}\right| \\
& +2 S(\vec{\beta}, \vec{\alpha} ; \vec{\gamma})+d(\vec{\beta}, \vec{\alpha}, \vec{\gamma})+S(\vec{\beta}, \vec{\alpha}),
\end{aligned}
$$


where

$$
d(\hat{\sigma}):=\frac{1}{4} \int_{\hat{N}} c^{2}(\hat{A})-\frac{1}{4}(2 \chi(\hat{N})+3 \operatorname{sign}(\hat{N})) .
$$

Note that $d(\hat{\sigma})$ is precisely the expression computing the virtual dimensions of Seiberg-Witten moduli spaces on closed manifolds.

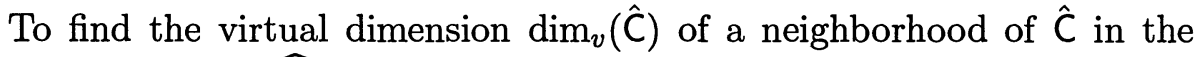
entire moduli space $\widehat{\mathfrak{M}}$ we only have to add the dimensions of the asymptotic limit sets $\operatorname{dim} \mathfrak{M}_{\kappa, n}=2 \operatorname{deg}\left|\mathbb{L}_{\mathbb{C}}\right|$ (recall that we have assumed $n<0$ ).

$$
\begin{aligned}
\operatorname{dim}_{v}(\hat{\mathrm{C}})= & d(\hat{\sigma})-\frac{1}{2}+\varepsilon(\ell)+\frac{\operatorname{sign}(\ell)}{4}-\frac{\ell}{4}+\operatorname{deg}\left|\mathbb{L}_{\mathrm{C}}\right| \\
& +2 S(\vec{\beta}, \vec{\alpha}, \vec{\gamma})+d(\vec{\beta}, \vec{\alpha}, \vec{\gamma})+S(\vec{\beta}, \vec{\alpha}) .
\end{aligned}
$$

We can now define the boundary correction term

$$
\begin{aligned}
\omega([\mathrm{C}]):= & -\frac{1}{2}+\varepsilon(\ell)-\frac{\ell-\operatorname{sign}(\ell)}{4}+2 S(\vec{\beta}, \vec{\alpha}, \vec{\gamma}) \\
& +d(\vec{\beta}, \vec{\alpha}, \vec{\gamma})+S(\vec{\beta}, \vec{\alpha})+\operatorname{deg}\left|\mathbb{L}_{\mathrm{C}}\right|,
\end{aligned}
$$

where $[\mathrm{C}] \in \mathfrak{M}_{\kappa, L}$, where $L \rightarrow \Sigma$ is a $V$-line bundle in $R_{\kappa}$ with singularity data $\vec{\gamma}$. The case when the asymptotic limit C satisfies $\nu(\mathrm{C})>0$ (that is $\left.\phi_{+} \equiv 0\right)$ can be safely left to the reader. The only changes in $\omega(\mathrm{C})$ occur at the term $\operatorname{deg}\left|\mathbb{L}_{\mathrm{C}}\right|$ above which should be replaced with $\operatorname{deg}\left|K-\mathbb{L}_{\mathrm{C}}\right|$.

When the boundary of $\hat{N}$ has several components (all Seifert manifolds of various types) then the asymptotic limit of a finite energy solution on $\hat{N}$ is a collection of solutions of the Seiberg-Witten equations on each of the components. The corresponding correction term is the sum of the correction terms determined by each component.

We conclude by considering the case when $\hat{C}$ is an irreducible finite energy solution on $\hat{N}$ with asymptotic limit $\mathrm{C}=\mathrm{C}_{\infty}$ a nondegenerate reducible. We argue exactly as in [N2]. Set $\rho=\rho(L)$ as in $\S 1.2$ and denote by $L_{\Sigma}=L(c, \vec{\gamma})$ the canonical representative of $L$.

The convergence to such a nondegenerate reducible continues to be exponential (see [MMS]) and thus we can use the same functional framework as above. Assume for simplicity the boundary has only one component. We distinguish two cases.

- $\rho(L) \neq 0$. 
We have to compute the $A P S$ index of a new operator $\hat{\mathcal{O}}_{w}$ which along the neck has the form

$$
\hat{\mathcal{O}}_{w}=\text { something } \times\left(\nabla_{t}-\mathcal{O}_{w}\right)
$$

where this time

$$
\mathcal{O}_{w}\left[\begin{array}{c}
\dot{\psi} \oplus \mathbf{i} \dot{a} \\
\mathbf{i} f
\end{array}\right]=\left[\begin{array}{cc}
D_{A} \dot{\psi} & \\
& -\mathbf{i} * d \dot{a}+\mathbf{i} d f \\
& \mathbf{i} d^{*} \dot{a}-2 w \mathbf{i} f
\end{array}\right] .
$$

(The spinor part $\phi$ of the asymptotic limit $[\mathrm{C}]=[\phi, A]$ is zero and thus $\mathcal{P}_{\phi} \equiv 0$.) Thus

$$
\operatorname{ind}_{\text {aps }}\left(\hat{\mathcal{O}}_{w}\right)=\operatorname{ind}_{\text {aps }}(\mathcal{N})-S F\left((1-t) \mathcal{O}_{0}+t \mathcal{O}_{w}\right) .
$$

The spectral flow contribution is easy to determine. The only eigenvalue of $\mathcal{O}_{t w}$ contributing to the spectral flow is $-\left.2 w t\right|_{t=0}$ with a single eigenfunction $\dot{\psi} \oplus \mathbf{i} \dot{a} \oplus \mathbf{i} f$, where $\dot{\psi}=0, \mathbf{i} \dot{a}=0$ and $f \equiv 1$. Hence

$$
\operatorname{ind}_{\text {aps }}\left(\hat{\mathcal{O}}_{w}\right)=\operatorname{ind}_{\text {aps }}(\mathcal{N})+1 \text {. }
$$

The index of $\mathcal{N}$ can be determined as above using instead the eta invariant of the adiabatic operator coupled with the flat connection $A \in \mathcal{R}(L)$ determined in $\S 1.2$. The nondegeneracy condition also implies $\operatorname{ker} D_{A}=0$. The eta invariant of $D_{A}$ is twice its $\xi$-invariant. Since $\operatorname{dim}_{\mathbf{R}} \tilde{\mathfrak{H}}_{0}=b_{0}(N)+b_{1}(N)=$ $1+2 g$ we deduce from (1.34)

$$
\begin{aligned}
\operatorname{ind}_{A P S}\left(\hat{O}_{w}\right)= & 1-(\chi(\hat{N})+\operatorname{sign}(\hat{N})) / 2+\frac{1}{4} \int_{\hat{N}}\left(c^{2}(\hat{A})-\mathbf{L}\left(\hat{\nabla}^{0}\right)\right) \\
& -\frac{1}{2}(2 g+1)-\frac{1}{2}(\operatorname{deg} K-\operatorname{deg}|K|)(1-2 \rho)+\ell \rho(1-\rho) \\
& -\frac{\ell}{6}-m \rho+2 S_{\rho}(\vec{\beta}, \vec{\alpha}, \vec{\gamma})+F_{\rho}(\vec{\alpha}, \vec{\beta}, \vec{\gamma}) .
\end{aligned}
$$

Again we have

$$
\int_{\hat{N}} \mathbf{L}\left(\hat{\nabla}^{0}\right)=\operatorname{sign}(\hat{N})+\frac{\ell}{3}-\operatorname{sign}(\ell)-4 S(\vec{\beta}, \vec{\alpha}) .
$$

We deduce

$$
\begin{aligned}
\operatorname{ind}_{A P S}\left(\mathcal{O}_{w}\right)= & d(\hat{\sigma})+\frac{1-2 g}{2}-\frac{\ell-\operatorname{sign}(\ell)}{4}+\ell \rho(1-\rho)-m \rho \\
& -\frac{1}{2}(\operatorname{deg} K-\operatorname{deg}|K|)(1-2 \rho) \\
& +2 S_{\rho}(\vec{\beta}, \vec{\alpha}, \vec{\gamma})+F_{\rho}(\vec{\alpha}, \vec{\beta}, \vec{\gamma})+S(\vec{\beta}, \vec{\alpha}) .
\end{aligned}
$$


To obtain the virtual dimension of a neighborhood of $\hat{C}$ in the entire moduli space we need to add the dimension of a neighborhood of $C_{\infty}$ inside the reducible torus and subtract the dimension of its isotropy group. This leads to a correction by $2 g-1$ and we deduce

$$
\operatorname{dim}_{v}(\hat{\mathrm{C}})=d(\hat{\sigma})+\omega_{\text {red }}(\mathrm{C})
$$

where

$$
\begin{aligned}
\omega_{r e d}(\mathrm{C})= & \frac{2 g-1}{2}-\frac{\ell-\operatorname{sign}(\ell)}{4}+\ell \rho(1-\rho)-m \rho \\
& -\frac{1}{2}(\operatorname{deg} K-\operatorname{deg}|K|)(1-2 \rho) \\
& +S(\vec{\beta}, \vec{\alpha})+2 S_{\rho}(\vec{\beta}, \vec{\alpha}, \vec{\gamma})+F_{\rho}(\vec{\alpha}, \vec{\beta}, \vec{\gamma}) .
\end{aligned}
$$

- $\rho=0$.

In this case we should use the first part of Proposition 1.10. A computation as above leads to

$$
\omega_{r e d}(\mathrm{C})=\frac{2 g-1}{2}-\frac{\ell-\operatorname{sign}(\ell)}{4}+2 S(\vec{\beta}, \vec{\alpha}, \vec{\gamma})+d(\vec{\beta}, \vec{\alpha} ; \vec{\gamma})+S(\vec{\beta}, \vec{\alpha}) .
$$

\section{Applications.}

\subsection{Tunnelings.}

We want to apply the virtual dimension formula formula to the special situation of tunnelings. Consider a Seifert fibration $N$ with oriented Seifert invariant $(g ; \ell, \vec{\alpha}, \vec{\beta})$. In this case $\hat{N}=\mathbb{R} \times N$ has two boundary components $N_{ \pm}:=\partial_{ \pm} \hat{N}=\{ \pm 1\} \times N$ which have opposite orientations. Denote by $\left(g ; \ell_{ \pm}, \vec{\alpha}_{ \pm}, \vec{\beta}_{ \pm}\right)$the oriented Seifert invariant of $N_{ \pm}$. We identify $N$ with $N_{+}$ and thus we have the following identities

$$
\ell_{-}+\ell_{+}=0, \quad \vec{\alpha}_{ \pm}-=\vec{\alpha}, \quad \vec{\beta}_{+}=\vec{\beta}
$$

and

$$
\vec{\beta}_{-}=\vec{\alpha}-\vec{\beta}=\left(\alpha_{1}-\beta_{1}, \ldots, \alpha_{m}-\beta_{m}\right) .
$$

Consider a tunneling $\hat{C}$ with irreducible asymptotic limits

$$
\left[\mathrm{C}_{ \pm}\right] \in \mathfrak{M}_{\kappa_{ \pm}, L_{ \pm}}(N) .
$$


Set $n_{ \pm}=\nu\left(L_{ \pm}\right)$. Clearly $\kappa_{-}=\kappa_{+}=\kappa$. In [MOY] it is shown that

$$
\operatorname{sign}\left(n_{-}\right)=\operatorname{sign}\left(n_{+}\right) .
$$

Assume for simplicity that both $n_{ \pm}<0$. Set $k=\operatorname{deg}\left(L_{+}-\operatorname{deg} L_{-}\right) / \ell=$ $\left(n_{+}-n_{-}\right) / \ell$. Since $L_{ \pm} \rightarrow \Sigma$ pull back to the same line bundle we deduce that $k \in \mathbb{Z}$. In fact, $L_{+}=L_{-}+k \mathbb{L}_{0}$ (where $N$ is viewed as the unit sphere bundle of $\mathbb{L}_{0}$ ).

We can now use the equality (2.15) which gives the virtual dimension of the space of tunnelings between $\mathfrak{M}_{\kappa, n_{ \pm}}$Denote this dimension by $\tau\left(\kappa ; n_{-}, n_{+}\right)$. We have

$$
\tau\left(\kappa ; n_{1}, n_{2}\right)=\frac{1}{4} \int_{\hat{N}} c(\hat{\sigma})^{2}+\omega\left(\left[\mathrm{C}_{-}\right]\right)+\omega\left(\left[\mathrm{C}_{+}\right]\right) .
$$

The integral term can be computed via transgression exactly as in the third transgression formula in [N2] and we obtain

$$
\frac{1}{4} \int_{\hat{N}} c(\hat{\sigma})^{2}=\frac{n_{-}^{2}-n_{+}^{2}}{\ell} .
$$

Thus we get

$$
\tau\left(\kappa ; n_{-}, n_{+}\right)=\frac{n_{-}^{2}-n_{+}^{2}}{\ell}+\omega\left(\left[\mathrm{C}_{-}\right]\right)+\omega\left(\left[\mathrm{C}_{+}\right]\right) .
$$

The last formula can be made a little bit more transparent. Denote by $\vec{\gamma}_{ \pm}$ the isotropies of $L_{ \pm}$. If

$$
\vec{\gamma}_{-}=\left(\gamma_{1}, \ldots, \gamma_{m}\right)
$$

and $c=\operatorname{deg} L_{-}$then $\operatorname{deg} L_{+}=c+k \ell$ and the data $\vec{\gamma}_{+}$are determined by

$$
\vec{\gamma}_{+}=\left(\gamma_{1}^{+}, \ldots, \gamma_{m}^{+}\right) \text {where } \gamma_{i}^{+}=\alpha_{i} \cdot\left\{\frac{\gamma_{i}+k \beta_{i}}{\alpha_{i}}\right\}
$$

The correction term can be further simplified since $\ell_{-}+\ell_{+}=0, \varepsilon\left(\ell_{-}\right)+$ $\varepsilon\left(\ell_{+}\right)=1$ and $S\left(\vec{\beta}_{-}, \vec{\alpha}\right)+S\left(\vec{\beta}_{+}, \vec{\alpha}\right)=0$. We conclude

$$
\begin{aligned}
\tau\left(\kappa ; n_{-}, n_{+}\right)= & \frac{n_{-}^{2}-n_{+}^{2}}{\ell}+\operatorname{deg}\left|L_{-}\right|+\operatorname{deg}\left|L_{+}\right| \\
& +S\left(\vec{\beta}_{-}, \vec{\alpha}, \vec{\gamma}_{-}\right)+S\left(\vec{\beta}_{+}, \vec{\alpha}, \vec{\gamma}_{+}\right) \\
& +d\left(\vec{\beta}_{-}, \vec{\alpha}, \vec{\gamma}_{-}\right)+d\left(\vec{\beta}_{+}, \vec{\alpha}, \vec{\gamma}_{+}\right) .
\end{aligned}
$$


Numerical experiments show that the above formula is in perfect agreement with the alternative description in [MOY].

Suppose now that the limit $C_{+}$is a nondegenerate reducible. Set as above $n_{-}=\nu\left(C_{-}\right)$and denote by $\tau\left(C_{-}, C_{+}\right)$the virtual dimension of the space of tunnelings from the component of $C_{-}$to the component of $C_{+}$. We distinguish two cases.

Case A. $n_{-}<0$. Arguing as above we deduce

$$
\begin{aligned}
d\left(C_{-}, C_{+}\right)= & \frac{n_{-}^{2}}{\ell}+\varepsilon(-\ell)+g+2 S\left(\vec{\beta}_{-}, \vec{\alpha} ; \vec{\gamma}_{-}\right) \\
& +d\left(\vec{\beta}_{-}, \vec{\alpha} ; \vec{\gamma}_{-}\right)+\operatorname{deg}\left|\mathbb{L}_{C_{-}}\right|+\delta_{+}(\rho)
\end{aligned}
$$

where

$$
\begin{aligned}
\delta_{+}(\rho)= & \ell \rho(1-\rho)-m \rho-\frac{1}{2}(\operatorname{deg} K-\operatorname{deg}|K|)(1-2 \rho) \\
& +2 S_{\rho}\left(\overrightarrow{\beta_{+}}, \vec{\alpha}, \overrightarrow{\gamma_{+}}\right)+F_{\rho}\left(\vec{\alpha}, \overrightarrow{\beta_{+}}, \vec{\gamma}\right), \quad \text { if } \rho \neq 0
\end{aligned}
$$

and

$$
\delta_{+}(\rho)=2 S\left(\overrightarrow{\beta_{+}}, \vec{\alpha}, \overrightarrow{\gamma_{+}}\right)+d\left(\overrightarrow{\beta_{+}}, \vec{\alpha} ; \overrightarrow{\gamma_{+}}\right), \quad \text { when } \rho=0 \text {. }
$$

Case B. $n_{-}>0$. The dimension formula is obtained from the above by performing the change

$$
\mathbb{L}_{\mathrm{C}_{-}} \rightarrow K_{\Sigma}-\mathbb{L}_{\mathrm{C}_{-}} .
$$

Of course one should replace $\gamma_{+}$with the singularity data of $K_{\Sigma}-L$ but as explained in Remark 1.6 this has no effect on the eta invariants and thus the overall contributions of the intervening Dedekind-Rademacher sums is unchanged by such a substitution.

\subsection{Froyshov invariants of Brieskorn spheres.}

As in [MOY], we want to consider in some detail the special case of the Seifert homology spheres $\Sigma(a, b, c)$ where $a, b, c$ are pairwise coprime integers $\geq 2$. The variational theory for the Seiberg-Witten energy functional on these manifolds is as simple as one can hope for. In particular, using the tunneling information in the previous section and our knowledge of eta invariants we will be able to produce estimates for the Froyshov invariant of such homology 
spheres. We begin by surveying the geometric facts which will be relevant in the sequel.

$\Sigma(a, b, c)$ is a Seifert fibration over an orbi-sphere $S$. We orient it as the link of the complex singularity

$$
\left\{x^{a}+y^{b}+z^{c}=0 ;|x|^{2}+|y|^{2}+|z|^{2} \leq \varepsilon\right\} \subset \mathbb{C}^{3} .
$$

As such, its rational degree is negative $\ell=-\frac{1}{a b c}$ and its singularity data are

$$
\vec{\alpha}=(a, b, c), \vec{\beta}=\left(\beta_{1}, \beta_{2}, \beta_{3}\right)
$$

with $\beta_{i}$ determined by the congruence

$$
\frac{\beta_{i}}{\ell \alpha_{i}} \equiv 1\left(\bmod \alpha_{i}\right)
$$

The canonical line bundle $K_{S}$ has rational degree

$$
\kappa=1-\left(\frac{1}{a}+\frac{1}{b}+\frac{1}{c}\right) .
$$

Consider the "simplex"

$$
\Delta(a, b, c)=\left\{(x, y, z) \in \mathbb{Z}_{+}^{3} ; \frac{x}{a}+\frac{y}{b}+\frac{z}{c}<\frac{\kappa}{2}, x<a, y<b, z<c\right\} .
$$

To a point $\mathbf{p}=(x, y, z) \in \Delta(a, b, c)$ we associate a line $V$-bundle $L_{\mathbf{p}} \rightarrow S$ with $\operatorname{deg}\left|L_{\mathbf{p}}\right|=0$ and singularity data $(x, y, z)$. Define the "energy" of a point $\mathbf{p}$ by

$$
E(\mathbf{p}):=\left(\operatorname{deg} L_{\mathbf{p}}-\frac{1}{2} \operatorname{deg} K_{S}\right)^{2} / \ell=\nu\left(L_{\mathbf{p}}\right)^{2} / \ell .
$$

Using the description of the critical set of the Seiberg-Witten functional we deduce that to any $\mathbf{p} \in \Delta(a, b, c)$ we can associate a pair of irreducible solutions $\left(C_{+}(\mathbf{p}), C_{-}(\mathbf{p})\right)$ of identical energy $E(\mathbf{p})$ (we deliberately omitted the normalization constant in Fact4, §2.2). $C_{+}(\mathbf{p})$ corresponds to a holomorphic vortex on $L_{\mathbf{p}}$ while $C_{-}(\mathbf{p})$ corresponds to an antiholomorphic vortex on $K_{S}-L_{\mathbf{p}}$. Any irreducible solution belongs to such a pair. We will denote the unique reducible solution by $C_{0}$. The discussion at the end of $\S 1.2$ shows this is nondegenerate.

For each $\mathbf{p} \in \Delta(a, b, c)$ set

$$
n_{ \pm}(\mathbf{p})=-\tau\left(C_{ \pm}(\mathbf{p}), C_{0}\right)
$$


where $\tau$ is described in the previous subsection. The dimension formulæ coupled with the Serre duality for the Dedekind-Rademacher sums (Remark 1.6) show that

$$
n_{-}(\mathbf{p})=n_{+}(\mathbf{p}) .
$$

Now we can form two Laurent polynomials

$$
P_{a, b, c}^{ \pm}(T)=\sum_{\mathbf{p} \in \Delta(a, b, c)} T^{n_{ \pm}(\mathbf{p})}
$$

Note that according to (3.1) $P_{a, b, c}^{+}=P_{a, b, c}^{-}$. Set $P_{a, b, c}=P_{a, b, c}^{+}$. The polynomial $2 P_{a, b, c}$ can be regarded as the Poincare polynomial of the (irreducible) Seiberg-Witten-Floer homology of $\Sigma(a, b, c)$ (negative gradings are allowed). Theorem 10.1.1 of [MOY] shows it is an odd polynomial.

We include below a few examples

$$
\begin{aligned}
P_{2,3,5} & =0 \\
P_{2,3,7} & =T^{-1} \\
P_{2,3,11} & =T^{-1} \\
P_{2,3,13} & =T \\
P_{3,3,17} & =T \\
P_{3,5,7} & =T+T^{-1} \\
P_{3,5,11} & =T^{5}+T+T^{-1} \\
P_{3,5,13} & =T^{3}+T^{5}+T^{9} \\
P_{5,7,9} & =2 T+T^{3}+T^{7}+T^{9}+T^{25}
\end{aligned}
$$

Given a Laurent polynomial

$$
P(T)=\sum_{n \in \mathbf{Z}} a_{n} T^{n}
$$

we define its set of gaps $\Gamma(P)$ by

$$
\Gamma(P)=\left\{m \in \mathbb{Z}_{+} ; a_{-(2 m+1)}=0\right\}
$$

and set

$$
m(P)=\min \Gamma(P) .
$$

For $P=P_{a, b, c}$ the invariant $m(a, b, c):=m\left(P_{a, b, c}\right)$ coincides with the integer $m$ defined at the beginning of Section 3 in [Fr]. Denote by $Y_{a, b, c}$ the Froyshov invariant of $\Sigma(a, b, c)$ defined in $[\mathrm{Fr}]$. 
Theorem 3.1. For any Seifert homology sphere $\Sigma(a, b, c)$ we have the following inequality

$$
Y_{a, b, c} \leq Z_{a, b, c}:=8 m(a, b, c)+\mathbf{F}_{a, b, c}
$$

where $\mathbf{F}_{a, b, c}$ is the invariant $\mathbf{F}(\Sigma(a, b, c))$ defined in (1.36) and (1.37) at the end of $\S 1.2$.

Proof. We begin by briefly recalling the definition of the invariant $Y$. We describe only the case of $\mathbb{Z}$-homology spheres.

Suppose $N$ is a $\mathbb{Z}$-homology sphere. Fix an arbitrary metric $g$ on $N$. By suitably perturbing the Seiberg-Witten equations on $N$ using as usual a 1 -form $\nu$ on $N$ as perturbation parameter we can arrange that the energy functional has only one nondegenerate reducible solution and the irreducible solutions are isolated and nondegenerate. The resulting gradient flow on $\mathbb{R} \times$ $N$ can also be perturbed to be in a Morse generic situation.

Denote by $S$ the set of irreducible solutions and by $\theta$ the reducible one. For every $\alpha \in S$ denote by $i(\alpha, \theta)$ the dimension of the space of tunnelings from $\alpha$ to theta (if this space is nonempty). Define $m$ as the smallest nonnegative integer such that there are no tunnelings $\alpha \rightarrow \theta$ with $i(\alpha, \theta)=2 m+1$. Denote by $\eta_{d i r}$ the eta invariant of the Dirac operator on $N$ (obtained canonically from the Levi-Civita connection) and by $\eta_{s i g n}$ the eta invariant of the odd signature operator. Note that these eta invariants depend on $g$. Set

$$
Y(N, g):=8 m+4 \eta_{d i r}+\eta_{s i g n}
$$

and define the Froyshov invariant $Y(N)$ by

$$
Y(N)=\inf \{Y(N, g, \nu) ; g, \nu\} .
$$

From the definition it is clear that one can obtain upper estimates on $Y(N)$ as soon as one can produce a concrete example of a metric on $N$ and perturbation $\nu$ satisfying the required nondegeneracy conditions and moreover the quantities $m, \eta_{s i g n}$ and $\eta_{d i r}$ are explicitly computable.

We present below one such instance when $N=\Sigma(a, b, c)$. As metric on $N$ we use the deformation of the Thurston metric discussed in Remark 1.35. This is obtained by a rescaling along the fibers the Thurston metric so that the fibers become very short, of radius $\approx r \ll 1$. Denote this metric by $g_{r}$ and let $\mathbb{S}$ denote the spinor bundle associated to the unique spin structure on $N$. For any connection $A$ on $\operatorname{det} \mathbb{S}$ we can now construct two Dirac operators: the Levi-Civita induced $\mathfrak{D}_{A, r}$ and $D_{A, r}$ induced by the adiabatic connection 
on $T N$, as in Remark 1.13. Correspondingly we get two Seiberg-Witten equations: $S W_{r e g}^{r}$ formulated in terms of $\mathfrak{D}_{A, r}$ and the adiabatic equations $S W_{a d}^{r}$. Denote by $S_{r e g}(r)$ (resp. $S_{a d}(r)$ the set of irreducible solutions of $S W_{r e g}^{r}\left(\right.$ resp. $\left.S W_{a d}^{r}\right)$. $S W_{\text {reg }}$ has a unique reducible the obvious one. In [N1] we established the following facts.

Fact 1. $S_{a d}^{r}$ is independent of $r$. Denote this set by $S_{a d}$. This set is described at the beginning of this subsection.

Fact 2. $S_{r e g}^{r} \rightarrow S_{a d}$ as $r \rightarrow 0$ in any Sobolev norm defined in terms of the metric $g_{1}$. Moreover, the rates of convergence can be estimated effectively in terms of $r$.

Note that the hessians of $S W_{a d}^{r}$ at solutions $\alpha \in S_{a d}$ are invertible but the norms of their inverses depend upon the metric $g_{r}$. In [N1] we also established

Fact 3. The eigenvalues of these hessians are bounded away from zero by a constant independent of $r$.

The last fact implies that any $\alpha \in S_{a d}$ admits a neighborhood containing for each $r \ll 1$ an unique solution of $S W_{r}$ which must be nondegenerate. Thus, for $r \ll 1$, we have a bijection

$$
\phi_{r}: S_{r e g}^{r} \rightarrow S_{a d} .
$$

The map $\phi_{r}$ is also compatible with the gradings. To show this we need a bit more notations.

For any $\alpha \in S_{r e g}^{r}$ denote by $H_{r e g}(\alpha, r)$ the hessian of $S W_{r e g}^{r}$ at $\alpha$. Define $H_{a d}(\beta, r)$ similarly. Additionally, we have two hessians $H_{r e g}(\theta, r)$ and $H_{a d}(\theta, r)$ at the trivial solution $\theta$.

$\phi_{r}$ preserves the gradings if

$$
S F\left(H_{r e g}(\alpha, r) \rightarrow H_{r e g}(\beta, r)\right)=S F\left(H_{a d}\left(\phi_{r}(\alpha), r\right) \rightarrow H_{a d}\left(\phi_{r}(\beta), r\right)\right)
$$

and

$$
S F\left(H_{r e g}(\theta, r) \rightarrow H_{r e g}(\alpha, r)\right)=S F\left(H_{a d}(\theta, r) \rightarrow H_{a d}\left(\phi_{r}(\alpha), r\right)\right) .
$$

The equality (3.4) follows from the fact that $H_{r e d}^{r}(\alpha)$ is very close to $H_{a d}^{r}\left(\phi_{r}(\alpha)\right)$ and both operators are invertible. Hence

$$
S F\left(H_{r e d}(\alpha) \rightarrow H_{a d}\left(\phi_{r}(\alpha)\right)\right)=0 .
$$


To prove (3.5) it suffices to show that

$$
S F\left(H_{r e d}(\theta) \rightarrow H_{a d}(\theta)\right)=0 .
$$

This spectral flow was analyzed in $\S 2.3$ of [N2] and it was shown to be zero.

The upshot of the previous discussion is that in order to compute the ingredient $m$ in the definition of $Y\left(\Sigma(a, b, c), g_{r}\right)$ we may as well use the adiabatic Seiberg-Witten equations. We see that it coincides with $m(a, b, c)$. The inequality (3.2) is now obvious.

We now associate to any negative definite $\mathbb{Z}$-quadratic form $q$ an integer $\Theta(q)$ defined by

$$
\Theta(q)=\operatorname{rk}(q)+\max \{q(\xi, \xi) ; \xi \text { characteristic vector }\} .
$$

We list below a few properties of this invariant.

P1 $\Theta(q)$ is divisible by 8 .

This follows from the arithmetic properties of the characteristic vectors described e.g. in [Se].

P2 $\Theta(q) \leq \operatorname{rk}(q)$ with equality if and only if $q$ is even.

Indeed, one has equality above iff 0 is a characteristic vector i.e. $q$ is even.

P3 $\Theta(q) \geq 0$ with equality iff $q$ is diagonal.

This highly nontrivial result is proved in [E].

P4 If $q=q_{1} \oplus q_{2}$ and $q_{2}$ is even

$$
\Theta(q)=\Theta\left(q_{1}\right)+\Theta\left(q_{2}\right)=\Theta\left(q_{1}\right)+\operatorname{rk}\left(q_{2}\right) .
$$

P5 If $M$ is a 4-manifold with boundary a $\mathbb{Z}$-homology sphere $N$ and if the intersection form $q$ of $M$ is negative definite then

$$
\Theta(q) \leq Y(N)
$$

where $Y(N)$ denotes the Froyshov invariant of $N$.

This is Theorem 1 in $[\mathrm{Fr}]$. 
For every triple $(a, b, c)$ of pairwise coprime integers $\geq 2$ denote by $\Gamma_{a, b, c}$ the intersection form of the Hirzebruch-Jung plumbing (see [HNK] or [NR] for a definition) associated to the Brieskorn homology sphere $\Sigma(a, b, c) . \Gamma_{a, b, c}$ is negative definite and set

$$
\Theta_{a, b, c}:=\Theta\left(\Gamma_{a, b, c}\right) .
$$

Note that Theorem 3.1 and P5 imply the following inequality

$$
0 \leq \Theta_{a, b, c} \leq Y_{a, b, c} \leq Z_{a, b, c}
$$

The following topological result is now obvious.

Proposition 3.2. Suppose $\Sigma(a, b, c)$ is such that $Z_{a, b, c} \leq 0$. Then

$$
Y_{a, b, c}=Z_{a, b, c}=0 .
$$

Moreover, if $X$ is a 4-manifold with negative definite intersection form $q$ and $\partial X=\Sigma(a, b, c)$ then $q$ is diagonalizable.

Naturally, one may ask whether there exist $\Sigma(a, b, c)^{\prime} s$ with $Z=0$. We present below a few values of $Z$.

\begin{tabular}{||c|c|c|c||}
\hline$(a, b, c)$ & $\mathbf{F}$ & $8 m$ & $Z$ \\
\hline \hline$(2,3,5)$ & 8 & 0 & 8 \\
\hline$(2,3,7)$ & -8 & 8 & 0 \\
\hline$(2,3,11)$ & 0 & 8 & 8 \\
\hline$(2,3,13)$ & 0 & 0 & 0 \\
\hline$(2,3,17)$ & 8 & 0 & 8 \\
\hline \hline$(3,5,7)$ & 0 & 8 & 8 \\
\hline$(3,5,11)$ & 0 & 8 & 8 \\
\hline$(3,5,13)$ & 8 & 0 & 8 \\
\hline \hline$(5,7,9)$ & 0 & 0 & 0 \\
\hline
\end{tabular}

The periodicity displayed in the first part of the table is a manifestation of a more general result. 
Proposition 3.3. Denote by $Q_{k}, \mathbf{F}_{k}$ and respectively $Z_{k}$ the quantities $P_{2,3,6 k+1}, \mathbf{F}_{2,3,6 k+1}$ and respectively $Z_{2,3,6 k+1}$. Then

$$
\begin{aligned}
& Q_{k}=\left\{\begin{array}{rl}
j T^{-1} & , \quad k=2 j-1 \\
j T, & k=2 j
\end{array},\right. \\
& \mathbf{F}_{k}=\left\{\begin{array}{rl}
-8, & k=2 j-1 \\
0, & k=2 j
\end{array} .\right.
\end{aligned}
$$

In particular, $Z_{k}=0$ for all $k \geq 1$.

Sketch of proof. Let us first point out that a weaker version of (3.9) is established in [MOY]. Computing the Dedekind sums may in general be a very daunting task. The singularity data of $\Sigma(2,3,6 k+1)$ are

$$
\vec{\alpha}=(2,3,6 k+1) \text { and } \vec{\beta}=(1,1, k) .
$$

The "simplex" $\Delta(2,3,6 k+1)$ is easily described since

$$
\operatorname{deg} K_{S}=-\frac{5}{6}+\frac{6 k}{6 k+1}
$$

is extremely small. The points in $\Delta(2,3,6 k+1)$ have the form $(0,0, z)$ where $z$ is a nonnegative integer such that $z /(6 k+1)<\operatorname{deg} K_{S} / 2$. Moreover, the invariant $\rho$ of the unique line bundle over $\Sigma(2,3,6 k+1)$ is $1 / 2$.

The computation of the quantities $n_{ \pm}(\mathbf{p})$ and $\mathbf{F}$ reduces essentially to computing Dedekind-Rademacher sums of the form

$$
s(k, 6 k+1, r, 0) \text { and } s(k, 6 k+1, r,-1 / 2)
$$

where $r$ is a rational number. At this point the reciprocity law comes in very handy. Denote by $R$ the reciprocity function defined by (1.17) and (1.18). Then

$$
\begin{aligned}
s(k, 6 k+1 ; x, y)= & -s(6 k+1, k ; y, x)+R(k, 6 k+1 ; x, y) \\
= & -s(1, k ; y+6 x, x)+R(k, 6 k+1 ; x, y) \\
= & s(k, 1 ; x, y+6 x)-R(1, k ;, y+6 x, x)+R(k, 6 k+1 ; x, y) \\
= & ((k y+6 k x)) \cdot((y+6 x))-R(1, k ;, y+6 x, x) \\
& +R(k, 6 k+1 ; x, y) .
\end{aligned}
$$

Above we used the usual continuous fraction decomposition of $k /(6 k+1)$ which, from a computational point of view, is more efficient than the 


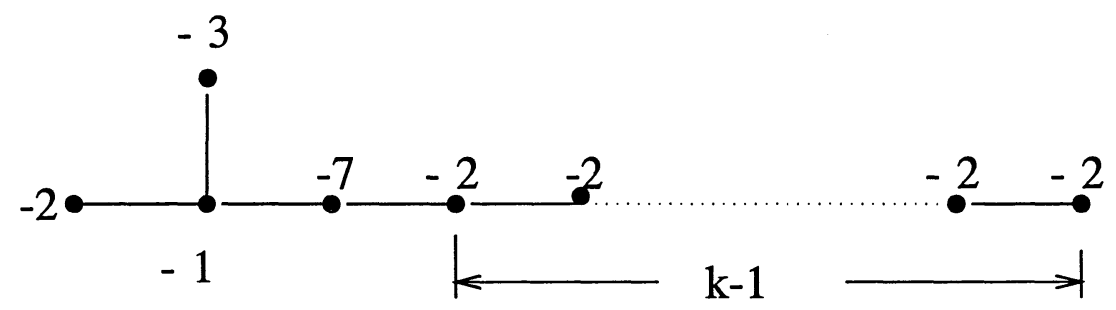

Figure 1: The plumbing diagram of $\Sigma(2,3,6 k+1)$

Hirzebruch-Jung continuous fraction decomposition. In this case the latter requires $k$ steps (see Figure 1). Obtaining the identities (3.9) and (3.10) is now an elementary, albeit tedious, accounting job.

From Proposition 3.3 and 3.2 we deduce immediately the following consequence.

Corollary 3.4. If $X$ is a smooth 4-manifold with negative intersection form bounding $\Sigma(2,3,6 k+1)$ then its intersection form must be diagonalizable.

Remark 3.5. The above corollary implies that $\Gamma_{2,3,6 k+1}$ is diagonalizable. This consequence is not entirely obvious but it can be proved directly. We present below a simple argument discovered in conversations with Ian Hambleton.

Set for simplicity $\mathcal{I}_{k}=\Gamma_{2,3,6 k+1}$. More explicitly, $\mathcal{I}_{k}$ is given by the plumbing diagram in Figure 1. Note that $\operatorname{rk}\left(\mathcal{I}_{k}\right)=4+k-1$. $\mathcal{I}_{1} \cong 4\langle-\mathbf{1}\rangle$ since all odd, unimodular, negative definite forms of rank 4 are so. Its matrix is

$$
A=\left[\begin{array}{cccc}
-1 & 1 & 1 & 1 \\
1 & -2 & 0 & 0 \\
1 & 0 & -3 & 0 \\
1 & 0 & 0 & -7
\end{array}\right]
$$

with inverse

$$
B=-\left[\begin{array}{cccc}
42 & 21 & 14 & 6 \\
21 & 11 & 7 & 3 \\
14 & 7 & 5 & 2 \\
6 & 3 & 2 & 1
\end{array}\right]
$$


The matrix of $\mathcal{I}_{k}$ can be written in block form

$$
A_{k}=\left[\begin{array}{cc}
A & L^{t} \\
L & N
\end{array}\right]
$$

where

$$
L=\left[\begin{array}{cccc}
0 & 0 & 0 & 1 \\
0 & 0 & 0 & 0 \\
0 & 0 & 0 & 0 \\
\vdots & \vdots & \vdots & \vdots
\end{array}\right]
$$

and

$$
N=\left[\begin{array}{cccccc}
-2 & 1 & 0 & 0 & 0 & \cdots \\
1 & -2 & 1 & 0 & 0 & \cdots \\
0 & 1 & -2 & 1 & 0 & \cdots \\
& & & & & \\
\vdots & \vdots & \vdots & \vdots & \vdots & \vdots
\end{array}\right]
$$

According to [HNK], Lemma 1.4 , the matrix $A_{k}$ is equivalent (as $\mathbb{Z}$-quadratic forms) to

$$
C=4\langle-1\rangle \oplus\left(-L B L^{t}+N\right) .
$$

A simple computation shows that

$$
L B L^{t}=\left[\begin{array}{cccc}
-1 & 0 & 0 & \cdots \\
0 & 0 & 0 & \cdots \\
& & & \\
\vdots & \vdots & \vdots & \vdots
\end{array}\right] .
$$

Thus $-L B L^{t}+N$ is the matrix of the intersection form $\langle-1 \mid k-2\rangle$ given by the diagram

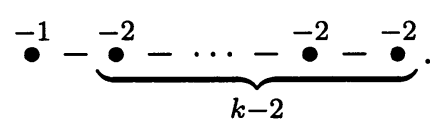

Using the same trick again, we can split off the isolated -1 in the above diagram and one can verify immediately the identity

$$
\langle-1 \mid m\rangle \cong\langle-1\rangle \oplus\langle-1 \mid m-1\rangle \text {. }
$$

This shows inductively that the form $\mathcal{I}_{k}$ is diagonal. 
Remark 3.6. As pointed out to the author by S. Akbulut and R. Fintushel, the Brieskorn spheres $\Sigma(2,3,13)$ and $\Sigma(2,3,25)$ bound contractible smooth manifolds and in these cases Corollary 3.4 follows from Donaldson's first theorem ([DK], Thm. 1.3.1). Also, as indicated by Nikolai Saveliev, $\Sigma(2,3,7)$ bounds a rational ball (with 2 -torsion in its first homology) and in this case the Corollary 3.4 also follows from Donaldson's first theorem.

The properties P1-P5 and Theorem 3.1 have another interesting topological consequence.

Proposition 3.7. Suppose $Z_{a, b, c} \leq 8$ and $X$ is a 4-manifold with the following properties.

(i) $\partial X=\Sigma(a, b, c)$

(ii) The intersection form $q$ of $X$ is negative definite and splits as $q=$ $q_{1} \oplus q_{2}$ where $q_{2} \neq 0$ is even.

Then $q_{2}=-E_{8}, q_{1}$ is diagonal and $Y_{a, b, c}=8$.

Warning. The above proposition does not prove $Z_{a, b, c}=8 \Rightarrow Y_{a, b, c}=8$. It only shows this is the cases provided $\Sigma(a, b, c)$ bounds a manifold satisfying (ii) above.

Proof. Indeed, from P4 we deduce

$$
\Theta\left(q_{1}\right)+\operatorname{rk}\left(q_{2}\right) \leq 8 .
$$

By P3 $\Theta\left(q_{1}\right) \geq 0$ so that $\operatorname{rk}\left(q_{2}\right) \leq 8$ which forces $q_{2}=-E_{8}$. In particular $\Theta\left(q_{1}\right)=0$ so that $q_{1}$ must be diagonal according to P3. Hence $\Theta(q)=8$ Using P5 and (3.8) we deduce

$$
8=\Theta(q) \leq Y_{a, b, c} \leq Z_{a, b, c} \leq 8 .
$$

We can also prove a counterpart of Proposition 3.3.

Proposition 3.8. Denote by $Q_{k}, \mathbf{F}_{k}$ and respectively $Z_{k}$ the quantities $P_{2,3,6 k-1}, \mathbf{F}_{2,3,6 k-1}$ and respectively $Z_{2,3,6 k-1}$. Then

$$
Q_{k}=\left\{\begin{aligned}
j T^{-1} & , \quad k=2 j \\
j T & , \quad k=2 j+1
\end{aligned}\right.
$$




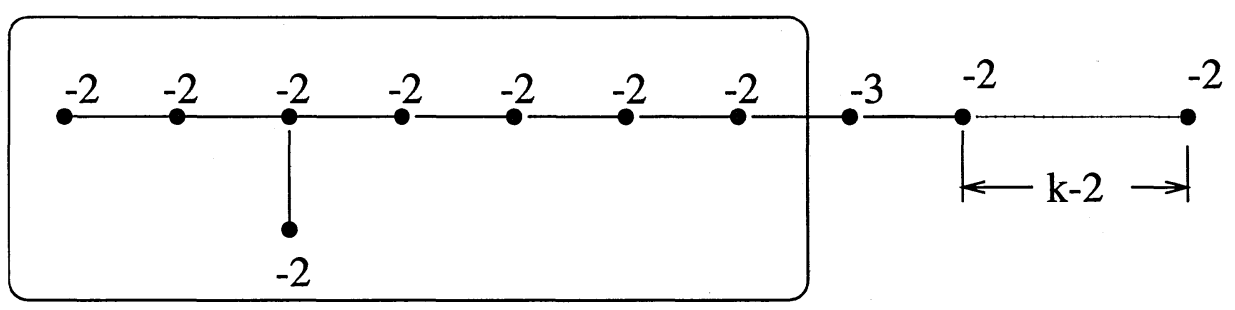

$-\mathrm{E}_{8}$

Figure 2: The plumbing diagram of $\Sigma(2,3,6 k-1)$

$$
\mathbf{F}_{k}=\left\{\begin{array}{ll}
8, & k=2 j+1 \\
0, & k=2 j
\end{array} .\right.
$$

In particular, $Z_{k}=8$ for all $k \geq 1$.

Proof. Follows the same lines as the proof of Proposition 3.3. The only difference now is in the Seifert invariants of $\Sigma(2,3,6 k-1)$. They are

$$
\vec{\alpha}=(2,3,6 k-1), \vec{\beta}=(1,2,5 k-1) .
$$

The Dedekind-Rademacher reciprocity law drastically reduces the computations using the following continuous fraction decomposition of $(5 k-1) /(6 k-$ 1)

$$
\frac{5 k-1}{6 k-1}=\frac{1}{1+\frac{1}{5-\frac{1}{k}}} .
$$

Corollary 3.9. (i) $Y_{2,3,6 k-1}=8$ for all $k \geq 1$.

(ii) If $\partial X=\Sigma(2,3,6 k-1)$ and the intersection form $q$ of $X$ is negative definite and splits as $q=q_{1} \oplus q_{2}, q_{2} \neq 0$ even, then $q_{1}$ is diagonalizable and $q_{2} \cong-E_{8}$.

Proof. To prove part (i) we will show that the Hirzebruch-Jung plumbing $X_{2,3,6 k-1}$ satisfies all the conditions in Proposition 3.7. Since $Z_{2,3,6 k-1}=8$ this will prove the equality $Y_{2,3,6 k-1}=8$.

The intersection form $\Gamma_{2,3,6 k-1}$ can be read from the plumbing diagram in Figure ??. Arguing as in Remark 3.5 we deduce that $\Gamma_{2,3,6 k-1}=\operatorname{diagonal} \oplus$ 
$\left(-E_{8}\right)$. Thus $Y_{2,3,6 k-1}=Z_{2,3,6 k-1}=8$. The second part of Corollary 3.9 is a special case of Proposition 3.7.

Remark 3.10. The case $k=1$ in the above corollary was first proved in [Fr].

Remark 3.11. Set

$$
d_{1}=0 \quad d_{n}=4+12+\cdots 8 n-12=4(n-1)(n-2)+4
$$

and define

$$
D_{n}(T)=\sum_{k=1}^{n} T^{d_{k}} .
$$

Numerical experimentations reveal a beautiful structure of the polynomials $P_{k}:=P_{2,4 k+1,4 k+3}^{+}$. More precisely they show

$$
P_{1}(T):=D_{1}(T)=T, \quad P_{k}(T)=P_{k-1}+T D_{k}(T) .
$$

Here are the first few of the polynomials $P_{k}$

$$
\begin{gathered}
T \\
T^{5}+2 T \\
T^{13}+2 T^{5}+3 T \\
T^{25}+2 T^{13}+3 T^{5}+4 T \\
T^{41}+2 T^{25}+3 T^{13}+4 T^{5}+5 T
\end{gathered}
$$

Numerical experimentations also show that $\mathbf{F}_{2,4 k+1,4 k+3}=0$ so that $Z_{2,4 k+1,4 k+3}=Y_{2,4 k+1,4 k+3}=0$. Thus, the only negative definite 4-manifolds which can bound $\Sigma(2,4 k+1,4 k+3)$ must have diagonalizable intersection form. One can give an alternative proof of this fact. More precisely, according to $[\mathrm{CH}]$ the Brieskorn manifold $\Sigma(2,4 k+1,4 k+3)$ bounds a contractible manifold $B(2,4 k+1,4 k+3)$. If $X$ is negative definite and bounds $\Sigma(2,4 k+1,4 k+3)$ then

$$
M=X \sqcup-B(2,4 k+1,4 k+3)
$$

is closed and negative definite and, by Donaldson's theorem, its intersection form is diagonal. A strikingly similar pattern is observed for the polynomials 
$P_{3,3 s+1,3 s+2}^{+}$. We list the first few of them $(s=1, \ldots, 4)$ and let the reader deduce the recurrence

$$
\begin{gathered}
T \\
T^{7}+2 T \\
T^{19}+2 T^{7}+3 T \\
T^{37}+2 T^{19}+3 T^{7}+4 T
\end{gathered}
$$

Again $\mathbf{F}_{3,3 k+1,3 k+2}=0$ so that $Y_{3,3 k+1,3 k+2}=0$. This agrees again with the conclusions of $[\mathrm{CH}]$ where it is established the spheres $\Sigma(3,3 k+1,3 k+2)$ bound contractible manifolds.

\subsection{Concluding remarks.}

The results we have established so far raise the following natural questions.

- Is it true that $Y_{a, b, c}=Z_{a, b, c}=\Theta_{a, b, c}$ for all $(a, b, c)$ ?

The inequality (3.8) shows the answer is positive provided one can establish only the purely number theoretic identity

$$
Z_{a, b, c}=\Theta_{a, b, c} .
$$

To answer this question a more manageable description of $Z_{a, b, c}$ and $\Theta_{a, b, c}$ (defined by (3.7)) is highly desirable.

- Remark 3.11 suggests the following question

Is it true that any $\Sigma(a, b, c)$ which bounds a smooth contractible manifold must have $Y_{a, b, c}=0$ ?

- Another number theoretic problem with possible interesting topological implications is the following. First define an equivalence relation on the space of negative definite $\mathbb{Z}$-quadratic forms as follos.

$$
q_{1} \sim_{s} q_{2} \Longleftrightarrow q_{1} \oplus m_{1}\langle-\mathbf{1}\rangle \cong q_{2} \oplus m_{2}\langle-\mathbf{1}\rangle
$$

for some nonnegative integers $m_{1}, m_{2}$.

Is it true that for any $\kappa \geq 0$ there exist only finitely many $\sim_{s^{-}}$ equivalence classes of negative definite $\mathbb{Z}$-quadratic forms $q$ satisfying $\Theta(q) \leq \kappa$ ? 
In [E] it is proved that the answer is positive if $\kappa<16$. Recently, M. Gaulter has shown in [Gau] that the result continues to hold for $\kappa \leq 24$. If the answer to the above question were positive this would mean that the invarinat $\Theta$ can be regarded as a "measure of complexity" of a negative definite quadratic form, i.e. the larger $\Theta(q)$ the further is $q$ from being diagonalizable. The equality $Y_{a, b, c}=Z_{a, b, c}=\Theta_{a, b, c}$ conjectured above would then mean that the Hirzebruch-Jung resolution is the "most complicated" negative definite 4-manifold bounding a given Brieskorn sphere!

- The Euler characteristic of the Seiberg-Witten-Floer homology of $\Sigma(a, b, c)$ (with the Thurston metric) is $2 P_{a, b, c}(-1)$. Following [C1-2] and $[\mathrm{KM}]$ define

$$
\alpha(a, b, c)=2 P_{a, b, c}(-1)-\frac{1}{8} \mathbf{F}_{a, b, c} .
$$

(The negative sign in front of $\mathbf{F}_{a, b, c}$ follows from our "orientation" of the Dirac operator which, although is the canonical one in the sense of $[\mathrm{BC}]$, it is not the one traditionally used in Seiberg-Witten-Floer theory.) Note that

$$
\alpha(2,3,6 k+1)=-k=\alpha(2,3,6 k-1)=-k .
$$

The calculations in [FSt] imply that

$$
\alpha(2,3,6 k \pm 1)=\frac{1}{8} \sigma(2,3,6 k \pm 1)=\lambda(2,3,6 k \pm 1)
$$

where $\sigma(a, b, c)$ denotes the signature of the Milnor fiber of $\Sigma(a, b, c)$ and $\lambda(a, b, c)$ denotes the Casson invariant of $\Sigma(a, b, c)$.

We now define for any oriented homology 3 -sphere $N$ and any good metric $g$ on $N$ (in the sense of [Mar]) the quantity

$$
\alpha(N, g)=\chi(N, g)-\frac{1}{8} \mathbf{F}(N, g)
$$

where $\chi(N, g)$ denotes the Euler characteristic of the Seiberg-Witten Floer homology of $(N, g)$ (defined in [Mar]) and

$$
\mathbf{F}(N, g)=4 \eta_{\text {dir }}(g)+\eta_{s i g n}(g) .
$$

The wall crossing formula of [Mar] and the variational formulæ for the eta invariants show that $\alpha(N, g)$ is independent of the metric and thus it is a topological invariant of $N$.

In $[\mathrm{KM}]$, P.Kronheimer and T. Mrowka conjectured that this invariant coincides with the Casson invariant and announced a proof of its validity 
when $N$ is a Brieskorn 3-sphere. In this case the conjecture is equivalent to an interesting number theoretic identity

$$
\frac{1}{8} \sigma(a, b, c)+2 P_{a, b, c}(1)=-\frac{1}{8} \mathbf{F}_{a, b, c} .
$$

The left hand side can be expressed in terms numbers of lattice points inside some convex regions of $\mathbb{Z}^{3}$ (see [FSt], [HZ]) while the right hand side is expressed in terms of Dedekind-Rademacher sums. In [N3] we prove the above identity (for arbitrary $a, b, c)$ using a lattice point count.

\section{References.}

[APS1] M.F. Atiyah, V.K. Patodi, and I.M. Singer, Spectral asymmetry and Riemannian geometry I, Math. Proc. Cambridge Philos. Soc. 77 (1975), 4369 .

[APS2] M.F. Atiyah, V.K. Patodi, and I.M. Singer, Spectral asymmetry and Riemannian geometry II, Math. Proc. Cambridge Philos. Soc. 78 (1975), 405432.

[APS3] M.F. Atiyah, V.K. Patodi, and I.M. Singer, Spectral asymmetry and Riemannian geometry III, Math. Proc. Cambridge Philos. Soc. 79 (1976), 71-99.

[BGV] N. Berline, E. Getzler, and M. Vergne, Heat Kernels And Dirac Operators, Springer Verlag, 1992.

[BC] J.M. Bismut and J. Cheeger, $\eta$-invariants and their adiabatic limits, Jour. Amer. Math. Soc. 2 (1989), 33-70.

[CH] A.J. Casson and J.L. Harer, Some homology lens spaces which bound rational homology balls, Pacific J. Math. 96 (1981), 23-36.

[C1] W. Chen, Casson invariant and Seiberg-Witten gauge theory, Turkish J. of Math. 21 (1997), 61-81.

[C2] W. Chen, Dehn surgery formula for Seiberg-Witten invariants of homology 3-spheres, dg-ga 9703009.

[DK] S.K. Donaldson and P.B. Kronheimer, The Geometry of Four-Manifolds, Clarendon Press, Oxford, 1990.

[E] N.D. Elkies, $A$ characterization of the $\mathbb{Z}^{n}$ lattice, Math. Res. Lett., 2 (1995), 321-326. 
[FL] M. Farber and J. Levine, Jumps of the eta-invariant. With an appendix by Shmuel Weinberger: Rationality of $\rho$-invariants, Math. Z. 223 (1996), 197-246.

[FSt] R. Fintushel, R. Stern, Instanton homology of Seifert fibred homology three spheres, Proc. London Math. Soc. 61 (1990), 109-137.

[Fr] K.A. Froyshov, The Seiberg-Witten equations and four manifolds with boundary, Math. Res. Lett., 3 (1996), 373-390.

[FS] M. Furuta and B. Steer, Seifert fibred homology 3-spheres and the YangMills equations on Riemann surfaces with marked points, Adv. in Math. 96 (1992), 38-102.

[Gau] M. Gaulter, Lattices without short characteristic vectors, Math. Res. Lett., 5 (1998), 353-362.

[JN] M. Jankins and W. D. Neumann, Lectures On Seifert Manifolds, Brandeis Lecture Notes, 1983.

[HS] P.J. Hilton and U. Stammbach, A Course in Homological Algebra, 2nd Edition, Springer-Verlag, 1997.

[HNK] F. Hirzebruch, W.D. Neumann, and S.S. Koh, Differentiable Manifolds and Quadratic Forms, Lect. Notes in Pure and Appl. Math., No. 4, Marcel Dekker, 1971.

[HZ] F. Hirzebruch and D. Zagier, The Atiyah-Singer Index Theorem and Elementary Number Theory, Math. Lect. Series 3, Publish or Perish Inc., Boston, 1974.

[Kaw] T. Kawasaki, The Riemann-Roch theorem for complex $V$-manifolds, Osaka J. Math., 16 (1979), 151-159.

[KK] P. Kirk and E. Klassen, The spectral flow of the odd signature operator and higher Massey products, Math. Proc. Cambridge Philos. Soc., 121 (1997), 297-320.

[KMO] P. Kronheimer, T. Mrowka, and P. Ozsvath, Dirac operators, holomorphic Euler characteristics and lattice points, preprint.

[KM] P. Kronheimer and T. Mrowka, Lectures at the 1st International Press Conference at U.C. Irvine, 1996.

[LM] R.B. Lockhart and R.C. McOwen, Elliptic differential equations on noncompact manifolds, Annali di Scuola Norm. Sup. di Pisa, (1985), 409-448.

[Mac] S. MacLane, Homology, Springer-Verlag, 1963. 
[Mar] M. Marcolli, Equivariant Seiberg-Witten-Floer homology, dg-ga 9606003.

[M] J.W. Morgan, The Seiberg-Witten Equations and Applications to the Topology of Smooth Manifolds, Mathematical Notes, Princeton University Press, 1996.

[MMR] J.W. Morgan, T. Mrowka, and D. Ruberman, The $L^{2}$-Moduli Space and a Vanishing Theorem for Donaldson Polynomial Invariants, International Press, 1994.

[MMS] J.W. Morgan, T. Mrowka, and Z. Szabó, Product formulas along $T^{3}$ for Seiberg-Witten invariants, Math. Res. Letters, 4 (1997), 915-929.

[MOY] T. Mrowka, P. Ozsvath, and B. Yu, Seiberg-Witten monopoles on Seifert fibered spaces, Comm. Anal. and Geom. 5 (1997), 685-791.

[NR] W.D. Neumann, F. Raymond, Seifert manifolds, plumbing, $\mu$-invariant and orientation reversing maps, in "Lecture Notes in Mathematics", vol. 644, 161-195.

[N1] L.I. Nicolaescu, Adiabatic limits of the Seiberg-Witten equations on Seifert manifolds, Comm. Anal. and Geom., 6 (1998), 331-392.

[N2] L.I. Nicolaescu, Eta invariants of Dirac operators on circle bundles over Riemann surfaces and virtual dimensions of finite energy Seiberg-Witten moduli spaces, math.DG/9805046, Israel J. Math., 114 (1999), 61-123.

[N3] L.I. Nicolaescu, Lattice points, Dedekind-Rademacher sums and a conjecture of Kronheimer-Mrowka, math.DG/9801030.

[O] M. Ouyang, Geometric invariants for Seifert fibered 3-manifolds, Trans. Amer. Math. Soc. 346 (1994), 641-659.

[Ra] H. Rademacher, Some remarks on certain generalized Dedekind sums, Acta Arithmetica, 9 (1964), 97-105.

[Sa] I. Satake, The Gauss-Bonnet theorem for V-manifolds, J. of the Math. Soc. of Japan, 9 (1957), 464-492.

[S] P. Scott, The geometries of 3-manifolds, Bull. London. Math. Soc. 15 (1983), 401-487.

[Se] J.P.Serre, A Course in Arithmetic, Graduate Texts in Mathematics, vol. 7, Springer Verlag, 1973.

[WW] E.T. Whittaker and G.N. Watson, A Course Of Modern Analysis, Cambridge University Press, 1940. 
RECeIVed OCtoBer 16, 1998.

UNIVERSITY OF NOTRE DAME

Notre DAme, IN 46556

E-mail address: nicolaescu.1@nd.edu 